\title{
Arithmetic Formulated Relevantly
}

\author{
Robert K. Meyer
}

Australian National University

\begin{abstract}
The purpose of this paper is to formulate first-order Peano arithmetic within the resources of relevant logic, and to demonstrate certain properties of the system thus formulated. Striking among these properties are the facts that (1) it is trivial that relevant arithmetic is absolutely consistent, but (2) classical first-order Peano arithmetic is straightforwardly contained in relevant arithmetic. Under (1), I shall show in particular that $0=1$ is a non-theorem of relevant arithmetic; this, of course, is exactly the formula whose unprovability was sought in the Hilbert program for proving arithmetic consistent. Under (2), I shall exhibit the requisite translation, drawing some Gödelian conclusions therefrom. Left open, however, is the critical problem whether Ackermann's rule $\gamma$ is admissible for theories of relevant arithmetic.

The particular system of relevant Peano arithmetic featured in this paper shall be called $R^{\sharp}$. Its logical base shall be the system $R$ of relevant implication, taken in its first-order form RQ. Among other Peano arithmetics we shall consider here in particular the systems $C^{\sharp}$, $\mathrm{J}^{\sharp}$, and $\mathrm{RM}^{\sharp}$; these are based respectively on the classical logic C, the intuitionistic logic J, and the Sobocinski-Dunn semi-relevant logic RM3. And another feature of the paper will be the presentation of a system of natural deduction for $\mathrm{R}^{\sharp}$, along lines valid for first-order relevant theories in general. This formulation of $\mathrm{R}^{\sharp}$ makes it possible to construct relevantly valid arithmetical deductions in an easy and natural way; it is based on, but is in some respects more convenient than, the natural deduction formulations for relevant logics developed by Anderson and Belnap in [ENT].
\end{abstract}

Australasian Journal of Logic (18:5) 2021, Article no. 4 


\section{I}

Why relevant arithmetic? There are several good reasons. Champions of relevant logics have long held the more familiar classical, intuitionist, and modal points of view to license fallacious arguments. (Cf. [ENT].) On the other hand, there has been a dearth of evidence that these fallacious arguments actually produce anything unsatisfactory. The most persistent dissatisfaction, in fact, has lain in the familiar license to pass from a contradiction to any statement whatsoever, which rules out non-trivial inconsistent theories. But no convincing example has been given, thus far, of a non-trivial inconsistent mathematical theory which we should take seriously; this defect will be remedied here.

And, more generally, there has not yet been a serious study of a concrete theory to determine the effect upon that theory of (1) the familiar fallacious argument pattern and (2) the converse insistence that relevantly fallacious arguments shall not be accepted. Most mathematicians, I think, would confess some intuitive dissatisfaction with, e.g., the claim that if $A$ is true then anything implies $A$. But it is also urged upon them, by the logicians who profer firm foundations for their discipline, that this claim (or some intuitive equivalent which has been prettied up not to sound quite so bad) is at least implicit and perhaps explicit in the reasoning that they have used to build up their subject.

Accordingly, it seems at least possible that mathematics (or other subjects to which logic is applied) will itself be mutilated if relevantly valid argument patterns are intuitively implausible. For, one supposes, our informal arguments do require relevance as a component of valid inference. So, in some sense of reasonableness, it does not seem reasonable that we should be unable to account formally for this component; to the contrary, it seems reasonable (and I shall argue here that it is reasonable) to assume insights into what mathematical reasoning, and mathematical structures, are really about.

Such, at any rate, is the informal motivation for this paper. Its formal motivation lies in the fact that arithmetic is indeed a subject worth studying from the relevant point of view, and that new light is indeed shed on the nature of deductive connections within formalized arithmetic by taking that point of view. But the reader is hereby warned also that the results of this paper remain partial; it is a first word in the relevant analysis of arithmetic, not a last word.

Australasian Journal of Logic (18:5) 2021, Article no. 4 
Two further points deserve mention. First, it is sometimes argued that relevant insights ought to be constructive, in a way that would satisfy (perhaps) a mathematical intuitionist. There is something to be said for this, since there are many points of resonance, both technical and philosophical, between constructivists and relevantists. But there are points of nonresonance also. First, the viewpoint taken in [ENT] is unabashedly nonconstructive, if not downright Platonistic. And, though I do not follow [ENT] into outright Platonism, I think that there are good reasons for retaining the non-constructive elements in relevant logics, especially insofar as they involve the analysis of negation. To be sure, if anyone wishes to pare down his relevant logic to the point where it is intuitionistically acceptable, it is quite easy to do so technically. But the price, in my opinion, is the loss of some of the most important features that go to make relevant logic attractive, both technically and philosophically.

For constructivism, as a doctrine in the philosophy of mathematics, may be taken as imposing either epistemological or ontological constraints on the size and shape of the mathematical Universe. And, very roughly, we may identify epistemological constraints as constraints on mathematical proof e.g., no theorem is proved unless it is proved constructively - and ontological constraints as constraints on the mathematical entities themselves - e.g., there are no mathematical entities save those as are generated constructively.

Brouwer's intuitionism, one must suppose, rests first of all on an ontological constructivism; for if proof is our instrument for coming to know what there is to know about mathematical entities, while the entities themselves are the free creations of the human spirit, then surely coming-to-know must at least satisfy the kinds of constraint that apply to entity-making. On the other hand, relevant insight, as presented in [ENT], need satisfy no ontological constraint whatsoever. Rather, in insisting upon relevance as a component of valid inference, it is clear that we are here dealing, mathematically, with an epistemological constraint only. What we know about the mathematical Universe shall not be established by fallacious arguments. Nor need it be presupposed that our knowledge has been obtained by fallacious arguments; what is fallacious, it might be urged, is rather the formal C-reconstruction of how we obtained that knowledge.

So, in particular, there is no need - and no good reasons - for the relevantist to deny, in virtue of his logical doctrines, such classically valid principles of reasoning as excluded middle and double negation, To be sure, he might have other grounds (e.g., having been born in Holland) for such denial. E.g.,

Australasian Journal of Logic (18:5) 2021, Article no. 4 
he might wish to impose ontological as well as epistemological constraints upon our exploration of the mathematical Universe. But such imposition is an added extra, which will, I think, commend itself only to a small minority of mathematicians, a group which, as a class, seems drawn to luxuriant theoretical ontologies as the sparks fly upward.

The principal relevant thrust, for better or for worse, has been different. It does not seek to constrain mathematicians, or any other Ontological Big Spenders, from their freewheeling ways. (At least not so far, though relevant set theory does seem likely to impose new restrictions on the kinds of comprehension axioms which are held to determine sets or properties. Such matters, however, are very much open at writing.) Rather, relevant systems were motivated by a view of what counts as a Good Argument. We believe, certainly, that most purported mathematical truths have been established by Good Arguments. So, since our doctrines concern correct formalization of the informal logic that we actually use, in mathematics as in other pursuits, it is to be expected in general that these doctrines will not upset but confirm common views about which theorems are true, if we take the common view as to which axioms are true. To be sure, there is always the chance that there is ome purported mathematical truth floating about which has been established by a Bad Argument; this chance, presumably, has been increasing as more and more mathematicians have been indoctrinated with the belief that $C$ is really the logic that they use. But I think it an off chance, on the whole.

Still, while still being underwhelmed by claims that relevant logics are (or ought to be) truly constructive, I do not want to be stuck, either, with the view that they offer just an alternative route to Old Wisdom. First, when one thinks relevantly, there is a question not only as to which formal theorems follow, but also as to which formal theorems express which intuitive theorems. In particular, we may think of the classical truth-functional vocabulary as well-suited for the expression of facts about the natural numbers, but as not oriented at all toward the expression of the laws in virtue of which we discover the facts.

Let me illustrate. The material $\supset$ I take to be a fact-laden connective; it merely transmutes simpler statements of fact into a more complex statement of fact. As such, it neither rests on nor licenses any lawful connections between the facts so compounded. But good arguments, whether deductively general or inductively particular, rest on connections, not facts.

The relevant $\rightarrow$, as I see it, is introduced precisely to take account of such 
connections. And this introduces, at the formal level, an opportunity to make distinctions which, though perhaps dimly present in the intuitive base, goes beyond it in sharpness and in formal clarity. For example, we should expect to find $A \supset B$ provable on some number-theoretic occasions when $A \rightarrow B$ is unprovable. And this happens. $0=2$, being arithmetically false, materially implies everything. But if we hypothetically overloaded arithmetic so as to identify 0 and 2, it's just gratuitous to suppose that we should thereby have identified, say, 0 and 1 . Indeed, when we take the integers modulo 2 , we $d o$ identify 0 and 2 , without going on to identify 0 and 1 . There's a moral there somewhere, which we shall proceed to draw below.

Ontological luxuriance escaped relevant criticism above. But its turn has now come. I allow myself the common arithmetical vocabulary below, and speak within it in wholly ordinary ways. (The reader, who is entitled to his own slants, philosophical and otherwise, may take that speaking as he will.) But natural numbers remain, on my views, funny objects, and I think it just Quinacious to suppose that, in employing the linguistic resources of English (or of Formalese) in ordinary ways we thereby commit ourselves to the existence of strange things. Mathematics is treasured among us (except for a small minority of the pure) for its utility in science and in daily life.

And, if we are to think relevantly about mathematics, what is to be hoped for most of all are not new routes to old truths but an expansion of the pragmatic imagination. Let us be free to wonder what it would be like if 0 were equal to 2 , and let us not be stopped short by our conviction that 0 isn't 2. For, to modify Russell's famous dictum, it is a pragmatically empty question Which are the mathematical entities, or Whether what we say about them is true. For our pragmatic Interest in mathematical theories lies in finding non-mathematical interpretations for them, and in general such interpretations are both partial and approximate. One is tempted to think that what this means is that they are really false; but what it really means is that mathematical theories are prized among us because they help us get on, while the question of their "real" truth or falsehood just doesn't arise; even less does the ontological status of, say, the number 318 arise when we say that the mass of Jupiter is 318 earth-masses.

I turn, now, to the second of our further points. It will occur, perhaps, to some readers to wonder whether the technical arguments of this paper are "relevantly valid," as one sometimes wonders whether arguments about intuitionist mathematics are mathematically acceptable to intuitionists.

These kinds of wonder, however, are not at all analogous. For the J-

Australasian Journal of Logic (18:5) 2021, Article no. 4 
partisan disagrees with most of the rest of us at the informal level, and indeed he has been notoriously reluctant to formalize his informal reasoning at all. But, at least, he is in revolt about what has passed previously as good mathematical reasoning - e.g., proving statements of mathematical existence by deriving a contradiction from assumptions of non-existence. So it makes very good sense to ask whether, in an informal study of an intuitionist formal system, the banned principles are admitted in our reasoning.

The motivation given in [ENT] for relevant logics could not stand in starker contrast. For, despite objections that the relevant logics lack the "disjunctive syllogism," or other components of intuitively valid reasoning, the relevant claim has not been that these intuitive principles are incorrect. To repeat, what is argued rather is that the formalization of our informal reasoning has been incorrect. To be sure, some argument-forms which the Cpartisan takes as valid are relevantly banned - e.g., the argument from $A$ and not- $A$ to $B$. But, while I certainly am at loggerheads with anyone who claims that he regularly uses that principle in his intuitive reasoning, this is not the claim which one hears. What one hears rather is that the C-formalization of our intuitive reasoning which licenses such argument forms is smooth and elegant (which is true); and that, while we never actually use the argument, it does no harm to have it in the system (which is false).

So what, after all, can one make of the question whether the informal arguments about $\mathrm{R}^{\sharp}$ are relevantly valid? Is this a question, say, as to whether the disjunctive syllogism is used? But why should it be avoided? There are, following [ENT], some formal counterparts of this informal argument form which are valid, whereas others are invalid. Obviously, if pressed, I should claim to be using a valid one.

And, to take the point a little further, the question about the relevant validity of the informal arguments here might be a question whether, if the entire discussion were formalized in a system based on $\mathrm{R}$, all the arguments would turn out valid. But that, too, is a question that begs the question. If the arguments were correctly formalized, of course they would turn out valid; for, as a partisan of $\mathrm{R}$ and a believer in the validity of my own informal arguments, I am compelled to hold that any formalization that makes them invalid is incorrect.

There is, I think, lurking in the background of discussions like this another presupposition, which rests on another begged question: Namely, that Cvalidity is the ordinary test of the validity of informal arguments. That claim, I hold, is evidently false, since there are invalid informal arguments 
which, when correctly formalized in C, turn out C-valid. But I do not expect my perception to alter the perceptions of the $\mathrm{C}$-partisan, who will beg the question his way just as surely as I will beg it my way.

Is there any way out of these "paradigm clashes"? Yes, there is; this paper is an exercise therein. For what I shall argue here is that there are pragmatic advantages in basing our formal arithmetic in $\mathrm{R}$, which do not exist if we base it on C. That is, I shall show that the fallacious informal argument patterns which the C-partisan endorses do in fact lead to formal trouble. His defense, after all, was that he does not use them, not that I was constricting his informal argument patterns so as to deprive him of a useful tool. Very well, then, I shall force him to use them, and then (I hope) I shall demonstrate to him that he would have been better off had he not used them. And that I am no worse off for having abjured them (though that demonstration remains incomplete, while the question of $\gamma$ is open).

In short, I am not going to fight with the C-partisan about what goes on in the "metalanguage." For we are unlikely to disagree about which informal argument forms, in use, are valid. What we would disagree about is how these argument forms are to be formalized. But that would just give us another formal system to talk about, in English. Again, we could formalize that talk, but eventually the escalator ride has got to stop. And it stops, always, in English (or other natural language), where the underlying question is always begged.

The solution, already presented, is not to ride escalators. If the R-partisan claims that he has a superior vehicle for formalizing informal reasoning, his first task is to find some informal reasoning and try to formalize it better. That is what is being attempted here. And it is a more or less objective question, when attacked with technical rigor, what the properties of a given formalized theory are. And, if we want a formalized theory to talk about, let us pick an interesting one, like arithmetic, rather than a dull one, like a formalized theory of talk about arithmetic. For, since we are informally more or less agreed, let us attend strictly to what we think are the formal counterparts of our intuitive arguments. Either we shall uncover some unpleasant anomaly (in the bad case) or find some fresh insight (in the good case) or both (in the usual case). So let us get on with our formal work, trusting good formalizations (e.g., of classical set theory, ZF) to produce nice theorems that reinforce motivation; and bad formalizations (ditto, NF) to be riddled with anomalies. By their (formal) fruits, ye shall know them. So, Calculemus.

Australasian Journal of Logic (18:5) 2021, Article no. 4 


\section{II}

The system $R^{\sharp}$ is formulated straightforwardly. It is based on a first-order language $\mathscr{L}^{\sharp}$ built up as usual from the countably infinite sequence of variables $x, y, z, x_{0}, x_{1}$, etc., the individual constant 0 , the unary operation ', the binary operations,$+ \times$, the binary predicate $=$, the unary connective $\sim$, the binary connectives $\wedge, \rightarrow$, and the universal quantifiers $\forall x, \forall y$, etc.

I prefer to view $R^{\sharp}$ (and other formal systems) as abstract in something approaching the sense of Curry's [FML]. This means that the constituents of $\mathscr{L}^{\sharp}$ are not to be taken as pieces of language in the ordinary sense (whatever that mixed-up ordinary sense may be, a point that Routley and I examine at some length in [ER]), but as abstract objects (obs, in Curry's framework) and functions. This frees the language we are using (Curry's U-language, which is here English with some special symbols adjoined) for its ordinary referential sense, to which the conventions of standard (mathematical) English will apply without further comment. In particular, the marks displayed above (e.g., ' $x$ ') are to be taken as names in English of certain formal objects. So ' $x$ ' names the first formal variable, namely $x$, just as Gerald Ford is named by 'Gerald Ford.' But $x$ is no more being displayed here than is Gerald Ford; indeed, since $x$ is taken as an abstract object (like the number 7 ) it cannot be displayed in principle; $\mathscr{L}^{\sharp}$, though I have stuck with tradition in calling it a "language," is by no means an "object language." (However, in resisting even the appearance of commitment here to a fallacious nominalism, with all the stilted and imperspicuous conventions which it is held to involve, I leave the reader free to make his own commitments. For, though the Curry-style plan makes for smoothness of exposition, which it is again Quinacious to think at all involved in any kind of ontological commitment whatsoever - we are just setting up our talk to use the linguistic resources of English conveniently, as does every mathematician (in his own tongue) - nothing substantial hangs on following this plan (save clarity). Indeed, I shall assume throughout that the reader knows well enough what a first-order language is, letting the algebraic details be taken as self-understood.)

We next build up the usual grammatical categories in $\mathscr{L}^{\sharp}$, as inductive classes. The class $\mathbf{F V}$ of formal variables has already been characterized. 0 is the only member of the class FI of formal individuals. The union of FV and FI is the class FA of formal atoms. From FA we generate, in a universally free way, the class $\mathbf{F T}$ of formal terms, using the operations ', + , and $\times$. I.e., these formal operations shall be taken as operations on FT, satisfying

Australasian Journal of Logic (18:5) 2021, Article no. 4 
an atom property which insures that all formal terms shall be built up from atoms, and a generalized ordered pair property, which says in particular that if $X+Y$ is the same term as $Z+W$ then $X$ is the same term as $Z$ and $Y$ is the same term as $W$, etc., and so which says in general that each term shall be uniquely constructed from its component atoms.

FT having been constructed, we build up the set AF of atomic formulas by doing more of the same, taking our formal predicate = as a binary function defined on $\mathbf{F T} \times \mathbf{F T}$ with range $\mathbf{A F}$, subject to analogous principles of universally free generation. I shall also refer to members of $\mathbf{A F}$ as (formal) equations. And a final step of universally free generation takes us from $\mathbf{A F}$ to the class FF of formulas of $\mathscr{L}^{\sharp}$ (to be identified here when convenient with $\mathscr{L}^{\sharp}$ itself); as a result, $\mathbf{F F}$ is closed under the formal connectives, among which I include not only $\rightarrow$ and $\wedge$, but also all the quantifiers $\forall x_{i}$, etc. (Thus, on the story here, $\forall x$ is just a unary operation on formulas, which does not contain $x$ as a constituent, and which is a different operation from $\forall y$. But I assume a 1-1 correspondence between quantifiers and variables, necessary for the usual story about freedom and bondage (just assumed here, not told), and letting the notation carry that correspondence.)

One thing about $\mathscr{L}^{\sharp}$ is that it doesn't have parentheses in it, or, for that matter, anything corresponding to an ill-formed formula. (Why should it?) Still, as presented, $\mathscr{L}^{\sharp}$ is hardly a break with tradition; indeed, I suspect that such kinds of presentation are coming to be the tradition, though accommodation to the pseudo-nominalism that underlay the old tradition dies hard.

We have now divided the formal objects that comprise $\mathscr{L}^{\sharp}$ into a number of (grammatical) categories. Just to recapitulate, these categories are FV, FI, FA, FT, AF, and FF; to these let us add explicitly the classes FO of formal operations, FP of formal predicates and $\mathbf{F C}$ of formal connectives (which would comprise respectively, on the story more often told, the (primitive) operation symbols, predicate symbols, and logical connectives and quantifiers).

Although our formal objects are themselves notation-free, we do need further notation in English for the purpose of discussing them. In particular, we need names for these formal objects. Happily, such names are supplied by standard mathematical convention; e.g., ' $0+x$ ' shall serve as the English name of the abstract formal term which one gets by applying the formal operation + to the formal individual 0 as first argument and the formal variable $x$ as second argument. Exactly so, were + ordinary addition and 
not our formal operation (which is decidedly not ordinary addition), might a mathematically inclined reader use ' $3+2$ ' as an English name for the number 5 .

As we have built up $\mathscr{L}^{\sharp}$, all our formal objects do receive unambiguous English names. For this purpose, we would ordinarily use parentheses, and such other devices of mathematical English as are necessary to render these names unambiguous. (For, since we are discussing formal objects which, in general, have been built up functionally from formal atoms, we need at least some notation which will indicate the order in which the functions are being applied, and what their arguments are. We also desire, naturally, that the notation should be as readable as possible.)

Grouping under parentheses is an unambiguous way of achieving our nominal goal. Nor need we be explicit about those conventions, since they are commonly understood. To achieve our second goal, clarity, we adopt the following rather familiar conventions. First, unary operations and connectives shall have minimal scope (consistent with the formal grammar). Second, the operation $\times$ shall bind more tightly than + . Third, binary connectives (including those to be defined) shall be ranked $\wedge, \circ, \vee, \supset, \supset, \rightarrow, \equiv, \equiv, \leftrightarrow$ in order of increasing scope, i.e., $\wedge$ binds more tightly than $\vee$, etc. Fourth, equal operations and connectives shall be associated to the left. Fifth, we use dots (sparingly) as parentheses in accordance with the conventions of Church's [IML] and Curry's [FML]. Finally, since none of our formal functions is defined on all formal objects, the reader is to be trusted to resolve further ambiguities in the direction of sense rather than nonsense, and to find the above conventions simple and relatively natural. Again, let him note merely that they are not conventions about omitting parentheses.

Another requisite is the provision of intuitive variables (Curry's $U$-variables) to range over our formal categories. This provision, in principle, will go like this: Natural numbers: $I, J, K$. Formal variables: $X, Y$, $Z$. Quantifiers: $\forall X, \forall Y, \forall Z$. Atomic formulas (equations): E. Formulas: $A, B, C$. I also use functional notation, in the manner of Kleene's [IMM], where proper substitution is at issue. E.g., where $T[X]$ is a term, $T[U]$ is the result of substituting the term $U$ for $X$ therein, throughout. Similarly, where $A[X]$ is a formula, $A[U]$ shall be the result of uniform substitution of the term $U$ for free $X$ therein, judiciously rewriting the bound variables of $A[X]$ on a definite plan if necessary to avoid capture of free variables of $U$ by outlying quantifiers of $A[X]$. (This notation, frankly, requires a certain contextual good will on the part of the reader; but its alternative tend to the 
imperspicuous, which is reason enough to prefer it.)

Natural numbers, it was just hinted, comprise a grammatical category of $\mathscr{L}^{\sharp}$. Why not? For, while the nominalistically syntactic tend to think of 0 , $0^{\prime}$, and so forth as their numerals, we Curry-style abstracters might just well think of them as the numbers. And so we define the inductive class $\mathbf{N}$ of natural numbers (which, from our viewpoint, is a subclass of FT) familiarly as follows:

$$
\begin{array}{ll}
D 0 . & 0={ }_{\mathrm{DF}} 0 \\
D 1 . & 1={ }_{\mathrm{DF}} 0^{\prime} \\
D 2 . & 2={ }_{\mathrm{DF}} 1^{\prime}
\end{array}
$$

and so forth. Note that, restricted to $\mathbf{N},{ }^{\prime}$ is the real operation of arithmetic successor; i.e., not only is it intended that it should be interpreted as such, but it is the operation. On the other hand, note that + and $\times$ are not the corresponding genuine arithmetical operations (for which we might use $\oplus$ and $\otimes$, just to keep the matter clear.) Indeed, though $\oplus$ is a genuine operation on $\mathbf{N}$, and it is quite true that $2 \oplus 2$ is the number 4 , it is not true that $2+2$ is the number 4 ; in fact, $2+2$ is not a number at all.

Though $2+2$ isn't a number, it is rather more like a number than is, say, $x+2$. So, $\mathbf{N}$ being in hand, let us define a further subset $\mathbf{Q N}$ of $\mathbf{F T}$, the quasi-numbers. I.e., let QN be the subset of FT that one gets by starting with 0 and closing under the formal operations ', + , and $\times$. Then, though quasi-number aren't numbers, they're the next best thing. E.g., $2+2$ is one of them.

Let us think about numbers and quasi-numbers just a little more. Where $I$ and $J$ are numbers, let the atomic formula $I=J$ be a numerical equation; the set of these we shall call NEQ. Where $I$ and $J$ are quasi-numbers, let $I=J$ be similarly a quasi-numerical equation, and let the set thereof be NEQQ.

Now NEQ and NEQQ play rather a privileged position with respect to the arithmetic that we all learned in school; in fact, at least to begin with, finding computational procedures to sort of the true from the false members

of these classes was our principal business. So, following a suggestion of Dunn, let us call a member of NEQQ correct if it is true according to those procedures, and incorrect otherwise. Then, surely, the least that we can expect of a formalized arithmetic is that it will deliver as theorems the correct quasi-numerical equations, and that it will deliver as non-theorems 
the incorrect ones. Moreover, we ought to be able to prove that fact in a very simple and highly constructive way. Who knows how confused we may be when (e.g., in postulating mathematical induction) we quantify over all the natural numbers together? Maybe, though one hopes not, we have confused ourselves into outright contradiction, which stares out at us from the Gödel result but which, at least thus far, has blinked when we are eyeball-to-eyeball with it.

But, however confused we may be, or however great our faith may be that we are really clear, we are not going to take back the arithmetic which we learned in first grade. $2+2=5$ is incorrect, and even the possibility (however $\operatorname{dim})$ that it is nevertheless a theorem of $C^{\sharp}$ should fill us with horror. Our rejection of it, in short, should be just as firm formally as it is informally. Sadly, on current stories, it is not.

We now add some further definitions, most of them familiar.

$$
\begin{array}{ll}
D \vee . & A \vee B={ }_{\mathrm{DF}} \sim(\sim A \wedge \sim B) \\
D \exists . & \exists X A=_{\mathrm{DF}} \sim \forall X \sim A \\
D \leftrightarrow . & A \leftrightarrow B=_{\mathrm{DF}}(A \rightarrow B) \wedge(B \rightarrow A) \\
D \supset . & A \supset B=_{\mathrm{DF}} \sim A \vee B \\
D \circ . & A \circ B=_{\mathrm{DF}} \sim(A \rightarrow \sim B) \\
D \neq . & T \neq U=_{\mathrm{DF}} \sim(T=U) \\
D \leq . & T \leq U=_{\mathrm{DF}} \exists X(T+X=U) \\
D<. & T<U=_{\mathrm{DF}} \exists X\left(T+X^{\prime}=U\right)
\end{array}
$$

We shall add more definitions below. Note, thus far, that $\rightarrow$, ०, and $\leftrightarrow$ are respectively relevant implication, fusion, and equivalence, whereas other connectives are classical. Note also that these definitions are presented schematically, and that they embody certain tacit conventions - e.g., in spelling out $D \leq$, we might specify that $X$ should be the first formal variable not free in $T$ or $U$. Such matters I leave henceforth to the reader's good sense.

Definitions, as just presented, can be taken in a dual sense. First, they may be taken merely as conventions about the use of English in referring to formal objects. Second, however, our definitions do call attention to other formal functions besides those set out explicitly in FO, FP, and FC. E.g., we may suppose that there is a formal function which, when applied to formulas $A$ and $B$, does yield the formula $\sim(\sim A \wedge \sim B)$ as value; and no harm comes from calling this function $\vee$, and from thinking of it as a formal connective

Australasian Journal of Logic (18:5) 2021, Article no. 4 
in an extended sense, with some but not all of the properties of the actual members of FC. (I.e., the atom property holds for it, and so, in a sense, does the generalized ordered pair property. But the latter does not hold in full, since it would rule out the existence of formal objects $A, B, C$ such that $A \wedge B$ and $\sim C$ are the same formal object, whereas this holds by definition of $\vee$, understood functionally.)

\section{III}

Our language $\mathscr{L}^{\sharp}$ having been set up, we now characterize the first-order theory $\mathrm{R}^{\sharp}$ in familiar axiomatic fashion. Following Kleene's [IMM], I divide the axioms and rules of $R^{\sharp}$ into three groups, depending on whether they are sentential (R-level), quantificational (proper RQ-level), or number-theoretic (proper $R^{\sharp}$-level).

As sentential axiom (schemes) for $\mathrm{R}$, the following suffice:

$$
\begin{array}{ll}
A 1 . & A \rightarrow B \rightarrow . B \rightarrow C \rightarrow . A \rightarrow C \\
A 2 . & A \rightarrow . A \rightarrow B \rightarrow B \\
A 3 . & A \wedge B \rightarrow A \\
A 4 . & A \wedge B \rightarrow B \\
A 5 . & {[A \rightarrow B] \wedge[A \rightarrow C] \rightarrow . A \rightarrow B \wedge C} \\
A 6 . & A \wedge[B \vee C] \rightarrow A \wedge B \vee A \wedge C \\
A 7 . & \sim \sim A \rightarrow A \\
A 8 . & A \rightarrow \sim B \rightarrow B \rightarrow \sim A \\
A 9 . & A \rightarrow \sim A \rightarrow \sim A
\end{array}
$$

I formulate rules, as before, by using $\&, \Rightarrow$ in a self-explanatory metalinguistic way. Rules at the sentential level of $R$ are

$$
\begin{array}{ll}
\rightarrow E . & A \rightarrow B \Rightarrow . A \Rightarrow B \\
\wedge I . & A \& B \Rightarrow . A \wedge B
\end{array}
$$

(This way of stating the rules is inspired by Belnap's requirement that the primitive rules for a system should correspond to theses of the system. And it illuminates, by the way, an important difference between $\rightarrow E$ and $\wedge I$, which finds its formal reflection in how natural deduction systems are set up

Australasian Journal of Logic (18:5) 2021, Article no. 4 
by Anderson and Belnap in [ENT].)

The quantificational level of $R Q$ is formulated economically with the following additional axiom scheme and rule.

A10. $\forall X A[X] \rightarrow A[T]$

$C \forall I . \quad A \rightarrow B \vee C \Rightarrow A \rightarrow \forall X B \vee C$, where $X$ is not free in $A$ or $C$

(The latter rule, a form of conditional universal generalization, appears to involve us in a violation of Belnap's criterion; it does not on the consideration that, intuitively, the premiss of the rule involves universal intuitive quantification over terms.)

As specific number-theoretic axioms, we take the following:

$$
\begin{array}{ll}
A 11 . & x^{\prime}=y^{\prime} \rightarrow x=y \\
A 12 . & x^{\prime} \neq 0 \\
A 13 . & x=y \rightarrow x^{\prime}=y^{\prime} \\
A 14 . & x=y \rightarrow x=z \rightarrow y=z \\
A 15 . & x+0=x \\
A 16 . & x+y^{\prime}=(x+y)^{\prime} \\
A 17 . & x \times 0=0 \\
A 18 . & x \times y^{\prime}=x \times y+x
\end{array}
$$

To these we add a rule of mathematical induction, namely

$$
R M I . \quad A[0] \& A[X] \rightarrow A\left[X^{\prime}\right] \Rightarrow A[X]
$$

$\mathrm{R}^{\sharp}$, which we may sometimes identify with its set of theorems, is the class of formulas of $\mathscr{L}^{\sharp}$ which is generated by the above axioms and rules.

Some motivation of $R^{\sharp}$, and comparison with other systems of first-order Peano arithmetic, is now in order. Relative to classical arithmetic $C^{\sharp}$ (as formulated, e.g., on p. 82 of [IMM]), $\mathrm{R}^{\sharp}$ may be viewed simply as the system which one gets by endorsing such sentential principles as are relevantly valid. For the property quantification and number-theory levels, at least so far as the postulates are concerned, are essentially unchanged. Relative to intuitionist arithmetic $\mathrm{J}^{\sharp}$ (again, as presented in [IMM]), the situation is more complicated. For here, at the sentential level $\mathrm{R}$ weakens $\mathrm{J}$ on the matter of 
implication, while $\mathrm{R}$ remains classical with respect to negation; relative to $\mathrm{J}$, the latter is of course a strengthening, though it is a strengthening that depends, as we shall see, on a different view of negation altogether.

Even so, the contrast with familiar systems, and in particular with $C^{\sharp}$, ought not to be overestimated. While I prefer, e.g., to have mathematical induction as a rule, both to simplify notation and because it seems to me, intuitively, to be the right form of mathematical induction, nothing is lost if we substitute instead its axiomatic strengthening, namely

$$
\text { A19. } A[0] \wedge \forall X\left(A[X] \rightarrow A\left[X^{\prime}\right]\right) \rightarrow \forall X A[X]
$$

For $A 19$ is provable in $\mathrm{R}^{\sharp}$, using $R M I$, whereas obviously the latter becomes a derivable rule when the former is taken as an axiom.

(There is, however, a subtle point involving the following classical equivalent of $A 19$.

$$
A 19^{\prime} . \quad \forall X\left(A[X] \rightarrow A\left[X^{\prime}\right]\right) \rightarrow . A[0] \rightarrow \forall X A[X]
$$

Taken intuitively, the sentential inference pattern underlying $A 19^{\prime}$ is something like

$$
\left(B \rightarrow B^{\star}\right) \wedge\left(B^{\star} \rightarrow B^{\star \star}\right) \wedge \ldots \rightarrow . B \rightarrow B \wedge B^{\star} \wedge B^{\star \star} \wedge \ldots
$$

As a particular finite instance of this, we might consider

(2) $B \rightarrow C \rightarrow B \rightarrow B \wedge C$

which is relevantly invalid on the ground that one would not use the fact that $B \rightarrow C$ to show, in general, that $B$ implies itself. So, while attending to relevance is to be presumed not to interfere with arguments by mathematical induction - such induction, certainly, is in appearance certainly a very relevant kind of argument form - our general motivating concern to pay careful attention to what is to be taken as the formal equivalent of a given intuitive principle is not to be upset on sloppy presuppositions of intuitive equivalence. Most of us, it is to be feared, have classical sentential calculus so firmly in our heads that there is a disposition to believe that we are giving $u p$ principles, when all in fact we are doing is being careful as to which are 
the principles.)

While we are on the subject of mathematical induction, another, and rather central, aspect of the general consistency problem for formal arithmetic is of course wrapped up right here. For built into formal principles like $R M I$ and $A 19$ is, of course, an intuitive comprehension principle, the effect of which on past experience we should beware of. For mathematical induction, as it is ordinarily understood, is not part of a full formal characterization of the natural numbers; rather, it is a consequence of that characterization. For, the characterization generally runs, "The natural numbers are just those entities that have every property possessed by 0 and its successors," or something quite similar. So believing in mathematical induction is just believing that all natural numbers have every property which 0 has and which is passed on under successor. And our formal induction principle $R M I$ goes beyond this claim if, perchance, there are some open formulas of $\mathscr{L}^{\sharp}$ which, in the systematic context, cannot reasonably, or perhaps even consistently, be taken to determine properties. Again, we all hope that this is an off chance. But it remains, nevertheless, a substantial assumption, leaving open the possibility that the $A[X]$ in $R M I$ ought to bear some restriction, consistent with a further relevant story about the natural numbers.

With that hedge against unsatisfactory inflation of the supply of theorems of formal number theories, the properly number-theoretic postulates of $\mathrm{R}^{\sharp}$ are firmly traditional. (In fact, they are adapted straight from [IMM], where the reader may find them all (taking induction in the $A 19$ form) in Kleene's postulate group B.)

Interest here centers, accordingly, on the question whether changing Kleene's logic changes significantly the supply of theorems that can be derived from these arithmetical postulates; and, if so, in what ways. On this point, against the view that relevant logics involve a radical restructuring of familiar notions, it should be noted that even our logical postulates are rather like Kleene's. (I have not, however, attempted to separate postulates as Kleene does. E.g., $A \rightarrow A$ is derivable here, but not from $A 1, A 2, \rightarrow E$ alone. And Kleene, who is working also with $\mathrm{J}$, needs specific $\vee$ and $\exists$ postulates, whereas here (as in the classical case) these are rendered superflous by $D \vee, D \exists$.)

In fact, the reader with [IMM] at hand will note that the chief omission here is of the postulate $1 a$, the logical "law" $A \rightarrow . B \rightarrow A$. We may look on $A 1, A 2$ as saving what is relevantly needed, in the present context, from Kleene's $1 a, 1 b$. $A 3, A 4$ correspond exactly to Kleene's $4 a, 4 b$, as $\rightarrow E$ does 
to Kleene's rule 2. $A 5$ saves what can be relevantly saved from Kleene's 4. $A 6$ must be added explicitly here. $A 7$ is Kleene's 8 , which in his context (but not here) collapses $\rightarrow$ to a material $\supset$. $A 8, A 9, \wedge I$ are again explicitly postulated here, though of course they are both $\mathrm{J}$-valid and C-valid. On the quantificational side, $A 10$ is exactly Kleene's 10 . And $C \forall I$ is a C-valid strengthening of the [IMM] rule 9, which produces here a classical rather than an intuitionist understanding of the quantifiers (in classical contexts). On the other hand, the Kleene postulates $1 b, 5 a, 5 b, 7,11,12$ are also derivable here, along with those already mentioned $(2,4 a, 4 b, 8,9,10)$, whereas 6 (like 3 ) holds in a form dual to $A 5$. And Kleene's number-theoretic postulates 13-21 are, as stated, exactly our postulates for $\mathrm{R}^{\sharp}$.

In short, and against the view that a battering reformulation of C-insights or J-insights is here involved, not much really has been changed. And even what has been changed survives in altered ways, as we shall see. In fact, the real changes can be summed up in the rejection of Kleene's axiom 1a, which licenses irrelevant deductive connections, and in the acceptance of $A 7$ and $C \forall I$, which sum up the relevant insistence on classical interpretation of classical logical particles.

\section{IV}

Having set up $R^{\sharp}$, we shall wish to prove some things in it, and then some things about it. To do so, I wish to set up, as promised, a general theory of natural deduction for relevant logics (and, for that matter, irrelevant ones). In doing so, I shall have in mind the natural deduction systems of [ENT], which stem from Anderson's [SCP] and which, in their vital implicational component, are motivated at length in the Anderson-Belnap [PCE].

Thinking naturally has been, from the first, an important motivating consideration for relevant logics. In addition to its cited import for the enterprises of Anderson and Belnap, it has also been studied relevantly by Prawitz, Urquhart, and Routley; and Urquhart in particular has made its analysis the keystone of his very important semantical work in relevant logics.

Nevertheless, there has always been a certain tension attending the relevant analysis of natural deduction. Put bluntly, it isn't necessarily very natural. While the underlying idea is quite clear-one tags formulas that appear in the course of a deduction with sets of numerals (or similar ornaments) in order to keep track of which conclusions depend upon (and hence

Australasian Journal of Logic (18:5) 2021, Article no. 4 
follow relevantly from) which hypothetical assumptions, the way in which the tags are attached has on occasion the appearance of being arbitrary. So, for that matter, do some of the inferences which are permitted (or which are disallowed) under certain taggings, especially when one begins to discriminate between systems (as is done slightly in [ENT], but which can be carried much further along lines explored by Urquhart and by Pottinger) by changing the rules. Add to all of this the binding of relevant systems of natural deduction in [ENT] to Fitch-style sub-proof methods (which, though these methods commend themselves eminently to Yalies and their logical descendants, are not so familiar in the Rest of the World, if any), and the sheer difficulty of remembering which tagging restrictions go with which rules, and a bit of rethinking appears in order.

Well, systems of Natural Deduction, however superficially attractive, have been a junkyard of ad hoc restriction, punctuated with intermittent discovery that, without such restriction, motivating intentions collapse. (Such discoveries, however, are not necessarily unpleasant, since they wipe out the used book market.) So one can hardly fault relevant logicians for going along with the trend. However, given a flood of distinct systems (C, J, R, E, T, B, etc.) that are all "naturally" motivated, one must begin to suspect that the "naturalness" of natural deduction is pretty much relative to the intuitions that one began with, however one dresses them up with assumptions and eliminations and instantiations and taggings and flagging and so forth.

In fact, as a general principle, it seems to me that the "natural" intuitions of formal logicians are themselves formal and sophisticated. Sometimes this leads to trouble; practice makes formal manipulation second nature, and we tend to lose sight of the first nature - namely, that we are reasoning beings, in a much looser sense of reason - out of which this second nature grew. More often, formal manipulation is an advantage, for it allows us to reconstruct with accuracy and precision the kinds of inferences on which we depended in our unwashed "natural" state.

But the logician's Nature is not "Nature, red in tooth and claw." The most common and persuasive "natural" arguments - the ad baculum, the ad hominem, the ad verecundiam, and so forth ad nauseam-have no standing among us, for our Nature is not only a rational one but a rationalized one, in the best sense of that now opprobious adjective. And rationalized Nature is unlikely to exist in us univocally; once formal intuition takes hold, we can bend system to purpose; and formal imagination takes the process a step further; once we have got accustomed to thinking systematically about

Australasian Journal of Logic (18:5) 2021, Article no. 4 
thought, new, and clearer, purposes arise.

So, as it seems to me, having a Natural Deduction formulation is a priori unlikely to offer independent motivation for a logic. What is "natural" from the point of view of a given formal system is to confine one's inferences, and to build one's non-logical theories, in ways that are licensed by the system, and to adapt one's notion of valid deduction to that a posteriori sort of naturalness. To be sure, the class of inferences to be considered valid can be stipulated in various ways - one can write down axioms, one can specify validity on certain semantical interpretations, and one can climb trees and make tables and employ a host of kindred devices. And there is often some surprise - and much motivating joy - when conceptually distinct devices turn out to yield the same class of valid inferences. But, thus viewed, so-called natural deduction systems are just one more device; and it is just a silly supposition that any such system incorporates a preferential way to tag and flag, which corresponds to what we would all do if we had never heard of Gentzen or Jaskowski or Quine or Curry or Rosser or Copi or Fitch or Lebanc or Anderson or Belnap or Mates or Thomason or Routley or Kalish or Montague or Prawitz or Kanger or Lambert or van Fraassen or Urquhart or Pottinger.

This does not mean that Natural Deduction is not worth pursuing; all of logic, one presumes, grows out of our desire to clarify and to regiment our "natural deductions." It is to say, however, that we are to look at systems whole, and not to get caught up or bogged down in particular devices through which they are stipulated. For, when one reads the fine print, the test of these devices is apt to be that they deliver wanted principles and fail to deliver unwanted ones, where it is on the whole both simpler and more economical to stipulate the wanted principles in some other fashion.

I do not wish to carry this last point too far. For in the work of Gentzen and of Prawitz in particular, using natural deduction techniques to stipulate systems produces some rather deep theorems, increasing in important ways our understanding and control of the systems in question. But here too systems may be fashioned to purpose, and generalized almost without limit. So the natural man, bearing his "natural logic," does not put in an appearance in this work either.

Still, as a class, natural deduction systems are over-advertised and undermotivated. Who among us, after all, has much improved his arguing capacity by learning Copi's (or anybody's) rules? What he is likely to have improved is his capacity to recognize the valid arguments and theses of Copi's (or 
anybody's) system. Do not misunderstand me; this is no small improvement. For the modern approach to logic bites off theories whole. And attending to the moves that can be made within theories is a way of becoming familiar with such theories, whether they be theories of pure logic or with intended application to some specific subject.

Below, the interplay between theories whole and move-making will prove to be delicate. For I shall see theories, and $R^{\sharp}$ in particular, as involving a certain order of ideas, which one expects their logical machinery to reflect. The delicate interplay, however, is not a function of the way in which a theory is stipulated; it is rather a function of the theory itself, both in the intuitive purposes which it reflects and in the actual formal machinery which reflects, adapts, and changes these purposes.

That our ultimate concern is with $\mathrm{R}^{\sharp}$ itself, and not with the mechanics of its stipulation, gives us a certain attitude toward its natural deduction formulations. Since I have little confidence in the independent motivational work of this or that technical device used in stipulating such formulationsand even less confidence in the formal evidence thus far adduced in favor of the independent motivational worth of the devices thus far employed in existing natural deduction formulations of relevant logics and theories - I shall introduce technical devices only insofar as they are rather directly reflected in the actual formal machinery of $\mathrm{R}^{\sharp}$ itself, or in some simple extension thereof.

And, above all, that I am concerned here with natural deduction in $\mathrm{R}^{\sharp}$ at all is motivated by the desire to exhibit formal demonstrations, or to make it easy for the reader to exhibit formal demonstrations by filling in trivial steps. For such purposes, as I have indicated, natural deduction is chiefly prized among us, and is taught to the freshmen so that they may do their exercises. For stipulating $\mathrm{C}$ as a natural deduction system is neither very simple nor very clear. The simple thing to do is to write down axioms; the clear thing to do is to characterize C (and afterwards CQ) semantically, beginning with truth-tables.

But neither of these courses inculcates the kind of practical familiarity that we need with $C$, and afterwards with $C Q$, in order to be at home with this system. Indeed, once we hit CQ, the "clear" semantical understanding tends to peter out, practically speaking. And even for $\mathrm{C}$, however simple the axiomatic method may be as a device for stipulating the theory, it tends not to be very helpful in enabling us actually to find formal demonstrations, and so to sort out accepted principles from rejected ones.

So the practical advantages of natural deduction are obvious after all. It 
is efficient. And it is certainly clear why it is efficient; by breaking complex wholes into easily manipulable parts, and by guiding our operations on parts by taggings and flaggings that inhibit impermissible manipulations, they yield a psychological ease and freedom which enables us to settle in minutes what we might otherwise have spent days trying to ascertain.

So, in revising [ENT] to produce a new natural deduction system for $\mathrm{R}^{\sharp}$, our objectives will be fourfold. First, we wish above all to facilitate deduction in $R^{\sharp}$ and in related systems. Second, we wish to stick closely to the formal machinery of $\mathrm{R}^{\sharp}$ itself. Third, we wish to maximize our capacity to use whatever we already know about $R$ and $R^{\sharp}$, from whatever source we may know it (including peeking ahead in this paper). Fourth, we wish our analysis to apply smoothly, mutatis mutandis, to related logics and theories.

The fourth point, though we won't dwell on it, will be automatically fulfilled. Every relevant logic (e.g., among those considered in [ALI]) can be given a natural deduction formulation, mutatis mutandis, along the lines set out here. So can most irrelevant ones. And, while we are content here to treat $\mathrm{R}^{\sharp}$ as a paradigmatic (regular) RQ-theory, exactly the same analysis may be adapted to all other such theories.

We shall dwell on the first three points. We shall also relate the treatment here to that of [ENT], and to other relevant studies of natural deduction. In particular, we shall show how motivational points urged elsewhere show up here. Or, in some cases, how and why they do not show up here.

There are also a number of points that we shall not dwell on (some of which, however, may be commended to the reader for further reflection). We are not interested here in any sort of independent formulation of $\mathrm{R}^{\sharp}$. $\mathrm{R}^{\sharp}$ was already stipulated in the last section, and there is no need to stipulate it again. Accordingly, there will be nothing like the syntactical completeness proofs offered by Anderson in [SCQ] and by Anderson-Belnap in [ENT]. Given our guiding principles, such a proof would be redundant. Similarly, since we are interested in maximizing the practical utility of our natural deduction techniques, we have no stake in economizing on the number of principles that are set out explicitly. While we shall set out enough principles that the reader may, if he wishes, prove even the axioms of $\mathrm{R}$ from them, using only a few of these principles, it is practically advantageous to make trivial moves (e.g., by double negation or DeMorgan laws) swiftly and trivally.

In opting thus for practicality, some interesting subjects are either obscured or left hanging. In its general outline, the present approach to natural deduction in relevant logics is very much influenced by the general theory of 
fusion (as developed, e.g., in [ALI] and [NAR]). It is influenced in another direction by Dunn's Gentzenization of $\mathrm{R}^{+}$, which appears under his name in $[\mathrm{ENT}]$. And it is negatively influenced by some recent claims that, on its "natural" motivation in [ENT], the system $\mathrm{R}$ is not quite right, in that there are impermissible maneuvers in the natural deduction system of [ENT] that should have been permitted, or permissible maneuvers that should have been forbidden. (Cf., for example, the papers by Urquhart and by Pottinger in [PRL].)

In fact, to the extent that we wish to attend at all to informal arguments whose ground is in taggings and flaggings, the claims of $\mathrm{R}$ are as good as any, and better than most. There is some point in attending; for [ENT] tags formulas so as to keep track of the premisses of an argument, so as to satisfy that relevance criterion which requires premisses actually to be used in a relevant derivation of the conclusion of that argument. Intuitively, this makes some sense, and we should attend to it, thus far. But, formally, what counts as use is itself a function of the tagging and tracking devices employed. I.e., it depends on the very system which this use-criterion is being used to motivate. So either the whole system must be related to a looser and more intuitive sense of use - and, for that matter, to other intuitive purposes that one had in mind in constructing the system, like making the Laws of Thought turn out true - or else the tracking procedure becomes viciously circular.

Now, with respect to the revisionist proposals at issue, they tend to accept crippling motivational loss for the sake of dubious technical gain. Central theorems in the theory of negation - e.g., excluded middle, double negation, or contraposition - are casually sacrificed for the sake of minor theorems about what one can do with disjunctions under hypotheses, while the underlying use-criterion becomes no clearer on the suggested revisions.

In fact, the real trouble lies with the [ENT] tagging techniques themselves. For R, they are without systematic significance, except via a certain kind of representation. (Algebraically, this representation, investigated in [IDP], relates commutative monoids to semilattices.) And this representation fails to extend in a natural way to accommodate the truth-functional connectives of $\mathrm{R}$, in particular $\vee$ and $\sim$. Proposals to change $\mathrm{R}$ rest on the insistence that this representation, not the intended meaning of the connectives of $R$, shall govern the stock of theorems of $\mathrm{R}$.

It is better to do things the other way round. The use-criterion which $R$ obeys is systematically clear; it is not further clarified, but is rather made more murky, by appeal to [ENT]-style scorekeeping devices. For this score- 
keeping becomes blatantly ad hoc just where the aforementioned representation becomes forced-i.e., on the truth-functions. But the representation itself (despite the semantical significance claimed for it by Urquhart in [SRL]) never did motivate R; at best it motivated only fragments of $\mathrm{R}$, and then only as a device for keeping track of the use-criterion which $\mathrm{R}$ itself respects.

I prefer here, and in general, to let $\mathrm{R}$, or any formal system worth taking seriously, speak for itself. For there is a gap between informal intuition and formal response thereto which just cannot be closed. Indeed, formal response often takes the form, as Belnap points out, "A good thing to mean by so-andso is such-and-such." Like all problems in ethics, value theory, ontology, and other branches of philosophy concerned with the Form of the Good, such claims must be weighed, both factually and normatively, for no single hot blast of Pure or Practical Reason settles them.

And that means, consciously, giving up a certain portion of what one took to be the original motivation of the relevant logics. That motivation rested heavily on the claim that relevance, as a component of valid inference, has now been accounted for. It was bad of $\mathrm{C}$ to leave it out; but now that we have $\mathrm{E}$, and $\mathrm{R}$, and their camp followers, it is now in. And that is good.

That we have $\mathrm{E}$ and crew is no doubt good; it has expanded our logical horizons. But the claim to have accounted for relevance will not, in an objective sense, stand scrutiny. For, very weak necessary conditions for relevance aside, the only sense in which [ENT] can be said to have characterized relevance is via the use criterion. But if the use criterion is itself relative to system - and in detailed formal fact it was never anything else - then it is not implausible to hold that every system intended seriously as a logic satisfies the use criterion, by its own lights.

In fact, I hold exactly that, with respect to the serious logics that I know of. Does $\mathrm{C}$, for example, have any trouble using $A \wedge \sim A$ to get to $B$ ? Of course not; there is a C-tautology in hand for the purpose. But this is not to say, as some C-apologists have had it, that there is never any trouble about getting from $A \wedge \sim A$ to $B$. No R-tautology smooths that general passage, for example.

Having thus viewed formal relevance as a system-dependent notion, not a system-independent notion, we seem to be heading back to the paradigm clash. But the way out is as before; we must view systems broadly, test them against their own motivation, and see what they are good for, and what they are not good for. Perhaps there is some good reason-other than the kind of hankering after the old days that would support any similar cause, like,

Australasian Journal of Logic (18:5) 2021, Article no. 4 
say, a Bourbon restoration - why people want to hold that $A \wedge \sim A$ relevantly implies $B$. For that's what $C$ appears to be committed to, making it just false that $\mathrm{C}$ has no concept of relevance. (But it leaves it arguable that $\mathrm{C}$ has no concept of the ridiculous.)

So our policy in choosing a natural deduction formulation for $\mathrm{R}^{\sharp}$ will be to set the R-principles which it respects out in the open, not to hide them under a cloud of technique. Let us think a bit about what this involves for any natural deduction system, of a familiar sort. Natural deduction typically proceeds by making hypotheses, drawing conclusions from these hypotheses according to specified rules, and then discharging (at least some of) the hypotheses according to further rules.

So, in general, we can divide the proof procedures of natural deduction systems into three categories: assumption procedures, which permit us to introduce hypotheses; working rules, which enable us to draw conclusions from the hypotheses; and discharge procedures, which rid of us the hypotheses that we have temporarily entertained. (In addition, there may be further procedures of a structural kind-e.g., a rule that allows us to reiterate a formula already derived, without doing any further work. Such rules have an auxiliary character, often associated with the kind of tracking devices associated with the system in question.)

Among the procedures, the central ones are the working rules. And the working rules tend to have systematic significance (often unlike the assumption and discharge procedures) quite apart from the peculiarities of natural deduction formulation. E.g., one expects a rule $\rightarrow E$ of modus ponens for $\rightarrow$ in a natural deduction formulation for R; nor does [ENT] disappoint us on this score. But $\rightarrow E$ is already an admissible rule of $\mathrm{R}$ itself; indeed, as above, it is standardly used to stipulate the set of theorems.

Now how is it, after all, that we can use $\rightarrow E$ after having made some assumptions (in natural deduction), just as we use it in grinding out theorems on no assumptions at all? How it is, in a certain sense, is that $\rightarrow E$ is the kind of rule, for familiar systems, that is invariant under assumptions. We are allowed, in short, to make that same move under assumptions that we would have been allowed to make under no assumptions.

It is also clear that invariance under assumptions is a tricky business. Evidently, to begin with, we don’t want all the admissible rules of $\mathrm{R}^{\sharp}$ to be invariant under assumptions. Substitution is a familiar case in point. And, in fact, it is sorting out the senses in which quantificational rules (e.g., of universal generalization) are invariant under assumptions from the senses in 
which they are not so invariant which provides employment and delight to makers of natural deduction systems for CQ and other logics. Such sorting, indeed, provides mush motivation (I mean "much motivation," but some typographical errors deserve to stand) for taggings and flaggings and trackings.

It might be thought that invariance under assumptions is a terrible business to keep straight. But in practice, and systematically, it is not so terrible at all. For, in the first place, deduction theorems for logical calculi give us clear systematic counterparts to a given stage of a natural deduction demonstration. Typically, for example, if $B$ has been deduced "naturally" from hypotheses $A_{1}, \ldots, A_{N}$ in a natural deduction formulation of a system $\mathrm{T}$, we shall have, as a theorem of $\mathrm{T}, A_{1} \wedge \ldots \wedge A_{N} \rightarrow B$. (We waive for the moment complications that attach to particular systems.)

Now, in the second place, replacement theorems for logical calculi tell us what we can do with the consequents of provable conditionals. Especially, saving the old antecedent, we can typically replace the consequent of a conditional with what it provably implies; all that we need to do this, indeed, is transitivity of provable implication.

So the soul of natural deduction, viewed systematically, lies in deduction theorems and in replacement theorems. For many simple working rules (e.g., a rule $\wedge E$ that tells us that we can go from $A \wedge B$ to $A$, or perhaps to $B$ if we'd rather), this is all that is involved. For, typically, $A \wedge B \rightarrow A$ is a theorem of the system, an appropriate deduction theorem holds, and provable implication is transitive. And this is all that one needs to justify systematically the assertion that a rule $\wedge E$ is invariant under assumptions.

Thus, in the first place, how we should formulate R-theories in general, and $R^{\sharp}$ in particular, in natural deduction style is a function of the replacement and deduction theorems that hold for R. We examine these matters in turn.

First, the replacement theorem with which we are concerned deals with replacement under provable implication. The weaker principle of replacement of equivalents (often taken, I think erroneously, as the replacement theorem) follows therefrom, but not conversely.

Second, the replacement theorem for implication is quite ordinary in relevant logics and theories. (I except, however, some very weak theories which Routley keeps pressing me to take seriously, on the contrary-to-fact claim that counterfactual logics are interesting; even for such emaciated theories, the main points here can be made, since the ordinary replacement theorem is not needed in full strength for our immediate purposes; but for all theories 
based on $\mathrm{R}$, and other standard relevant logics, that theorem holds in full strength anyway. In particular, it holds for $\mathrm{R}^{\sharp}$ as formulated above, which is all we need concern ourselves with here.)

Since the replacement theorem will be used here quite a lot, I shall recall for the reader its standard formulation. Let $A$ be any formula of $\mathscr{L}^{\sharp}$. To each occurrence of a subformula $B$ in $A$, we assign a charge, either + or -. this charge is assigned recursively, as follows. We begin with outermost subformulas.

(i) $A$ is positively charged in $A$

(ii) For each subformula of $A$ of the form $C \wedge D, C \vee D, C \circ D, B \rightarrow C$, $B \supset C, \forall X C, \exists X C$, each of $C, D$ have the same charge as the given subformula

(iii) For each subformula of $A$ of the form $B \rightarrow C, B \supset C, \sim B$, the charge on $B$ is the opposite charge to that of the given subformula.

I follow Schütte in using $A\left[B_{+}\right], A\left[C_{-}\right]$, and $A\left[B_{+}, C_{-}\right]$as shorthand for formulas $A$ with a specified positive occurrence of a formula $B$, with a specified negative occurrence of a formula $C$, or both, where $A\left[D_{+}\right], A\left[E_{-}\right]$, and $A\left[D_{+}, E_{-}\right]$specify the result of replacing $B$ with $D, C$ with $E$, or both in the specified occurrence(s) in $A$. Then

Directed Replacement Theorem for $\mathrm{R}^{\sharp}$. Let $B \rightarrow C$ be a theorem of $\mathrm{R}^{\sharp}$. Then if $A\left[B_{+}\right]$is a theorem of $\mathrm{R}^{\sharp}$, so is $A\left[C_{+}\right]$. If $A\left[C_{-}\right]$is a theorem of $\mathrm{R}^{\sharp}$, so is $A\left[B_{-}\right]$. If $A\left[B_{+}, C_{-}\right]$is a theorem of $\mathrm{R}^{\sharp}$, so is $A\left[C_{+}, B_{-}\right]$.

The proof of the theorem for $\mathrm{R}^{\sharp}$ proceeds on familiar lines (cf. [FML] or $[\mathrm{ENT}])$ and is omitted. It may be generalized in obvious ways, of which only the replacement of equivalents is worth noting.

Undirected Replacement Theorem for $\mathrm{R}^{\sharp}$. Let $B \leftrightarrow C$ be a theorem of $\mathrm{R}^{\sharp}$. Then any replacement of $B$ with $C$ in a theorem of $\mathrm{R}^{\sharp}$ yields a theorem of $\mathrm{R}^{\sharp}$ yields a theorem of $\mathrm{R}^{\sharp}$. (Proof immediate from the last theorem and elementary properties of $\leftrightarrow$.)

Before leaving the replacement theorems, 2 notational points are in order. First, we characterized charges with respect to defined as well as primitive logical particles. Strictly speaking, this characterization only applies to primitive particles, though it works out as indicated for defined ones. Second, our 
use of brackets in the replacement notation above is temporary; it is marked by the fact that notation for formulas and charges occurs inside the brackets. But the ordinary use of brackets, and substitution notation, continues here as before to mark the proper substitution for a variable $X$ in a formula $A[X]$ of a term $T$. This use is also notationally distinguished, and follows the $[\mathrm{IMM}]$ convention already set down.

Let us next examine the $\mathrm{R}^{\sharp}$ form of the other chief pillar of natural deduction, namely, the deduction theorem. This theorem might read, ordinarily, "If one can deduce $B$ from $A_{1}, \ldots, A_{n}$, then one can deduce $A_{n} \rightarrow B$ from $A_{1}, \ldots, A_{n-1}$." So put, however, the theorem is subject to two ambiguities. One of them, pointed out in [ENT], concerns the meaning of a "deduction from hypotheses." What, in the first place, counts as a deduction? And how, in the second place, must the hypotheses enter into that deduction so that $B$ is deduced from those hypotheses?

The second ambiguity is pointed out in [NAR]. When one has more than one hypothesis, how are multiple hypotheses to be compounded in characterizing deduction from those hypotheses? Put otherwise, what does the deducibility relation relate? Is it an $n+1$-ary relation, for variable $n$ ? Or is it a binary relation, between the hypotheses somehow collected together and what may be concluded from this collection? If the latter, what is the nature of the collecting process? Does one just make a list-i.e., form a sequence in which, e.g., the same hypothesis may occur repeatedly in different places? If so, does one care about the order in which the hypotheses are given? Or does one just collect the hypotheses into a set?

To be sure, ordinary precise descriptions of "deduction from hypotheses" resolve these ambiguities. Indeed, the ambiguities are resolved so crisply that vested interests arise in defense of the position that there were no ambiguities to begin with; that we all "know" what is properly to count as deductions from hypotheses.

Again, having undergone successful indoctrination is not to be confused with having thought clearly. The gap between informal intuition and its formal realization yawns once more, and it is hard to stile a yawn in the presence of tired arguments that there is only one way to fill the gap. Or that, even if it is not better to fill the gap in the customary way, whatever disturbs our "conceptual scheme" least is nonetheless preferable to fresh thought. There is something, as Belnap and Smart point out, to Quine's concern for "minimum mutilation." But, over the years, I personally have lost my appetite for that concern, especially where matters logical ("central" to the conceptual

Australasian Journal of Logic (18:5) 2021, Article no. 4 
scheme, and hence "highly immune" to revision) are involved. For the concern tends to confuse recent formalizing proposals - e.g., those made by the most eminent logicians, and adopted in the most popular texts - with the logical core of the conceptual scheme itself. Thus, even if one is conservative on general questions of conceptual revision, one ought not to confuse attachment to a received scheme with attachment to particular points of formal engineering. And, beyond that, I suspect that only a general ideological conservatism motivates a conservative outlook on conceptual issues anyway. It is a Stone Age mentality, most appropriate to Stone Age culture, which gives a priori preference to those ideas and notions that are entrenched. Respect for the Wisdom of the Ages is fine; respect for the Folly of the Ages is not. But there is, I suspect, no general principle - and, a fortiori, no fussy formalizing principle - that suffices to tell the one from the other.

So let us return to the gap between a loose informal statement of the deduction theorem, with its evident ambiguities, and the concrete proposals that have been made to fill that gap. And let us concentrate on the second ambiguity, for the moment. It is convenient, and I am here content, to take the deducibility relation as a binary relation between premisses, somehow collected, and a conclusion. But I am not content to suppose that there is some preferred collecting process, which is all-ways faithful to our understanding of the definite article when we hold that a valid argument is one whose conclusion follows from the premisses. Accordingly, I shall speak here, as I did in [NAR], of a bunch of premisses, and I shall not assume that there is any preferred way of bunching (e.g., by set formation).

Rather, in letting a system speak for itself, we should ask what devices are (or ought to be) offered by that system for bunching statements together, and why these devices exist for that purpose in the system in question. In relevant logics, and in $\mathrm{R}$ particularly, answering this question is facilitated by the presence of bunching connectives, which can be adapted exactly to this purpose of collecting premisses together.

In $\mathrm{R}$, in particular, there are two bunching connectives with clear motivational import. They are the ordinary conjunction $\wedge$ and the fusion connective o. (Motivation for the latter can be found in many places; e.g., in [NAR] and in [ENT].) These connectives, moreover, have distinct motivational importance. $\wedge$ is intended in the familiar extensional sense, and semantic investigations into $\mathrm{R}$ (e.g., [SEI], [NAR]) indicate that this is exactly the sense which $\wedge$ has in $R$.

Saying that $\wedge$ is extensional suggests that $\circ$ is "intensional," motivating

Australasian Journal of Logic (18:5) 2021, Article no. 4 
its classification as an "intensional conjunction" in a number of places. I now resist this classification, which I think misleading, for reasons set out in $[\mathrm{NAR}]$ and in $[\mathrm{ER}]$. In particular, "intensional" suggests a preoccupation with meanings, which is not being here essayed. Rather, let us be content on this point to follow eminent philosophical authority by attending to the use of the notion in question.

Attention to use is a formal matter as much as it is an informal matter. While we devise formal systems on a certain intuitive input, our systems very rapidly outrun this input. It is well that they should; formal systems, like natural laws, are most useful to us when they broaden our perspective beyond a simple recording of the data with which we began. We record, in either case, more than we know. And, to discover all that is involved in the formalizing process - not only in terms of intuitive input but also in terms of practical output - the hard work of getting clear on the systematic import of the components of a formal system is by all means necessary.

So $\circ$ and $\wedge$ work differently in $\mathrm{R}$. They correspond, respectively, to a tighter and a looser way to bunch premisses in argument. Specifically, $\circ$ bunches premisses in a way that inhibits the unbunching of these premisses; if $B$ follows from premisses bunched by $\circ$ in the system $\mathrm{R}$, then all of these premisses have been used in a relevantly valid way for the derivation of $B$. On the other hand, if the premisses have only been bunched by the looser connective $\wedge$, then some of the premisses may well have been superfluous for the derivation in question; i.e., we can unbunch, and still derive.

In particular, this means in $\mathrm{R}$ that $B$ follows from $A \wedge B$, but that it does not follow in general from $A \circ B$. And we are now returned to the former of our concerns above. What is a deduction from certain premisses? On R-insights, this question can be reduced to the question of the validity of a certain implication. For, having bunched our premisses into a formula $A$, it is held in $\mathrm{R}$ that $B$ is deducible from premisses so bunched just in case $A \rightarrow B$ is a theorem of $\mathrm{R}$. For that matter, it is so held in $\mathrm{C}$, in $\mathrm{J}$, and in almost every other reasonable system, save that the bunching principles are different.

Much more needs to be said on this topic, but it is not particularly in place to say it here. Suffice it, accordingly, to remark that the connective $\rightarrow$ of $\mathrm{R}$ is correlated (in a familiar way) with the bunching operator $\circ$. It is not correlated with the looser operator $\wedge$ (though we might, if we please, introduce a connective of an implicational kind corresponding to $\wedge$ as $\rightarrow$ does to $\circ$; depending on one's attitude toward negation, this connective might 
be of an intuitionist kind, as it is in Pottinger's studies of relevant natural deduction; or it might be outright (semantic) material implication, on lines explored by Routley and me and Urquhart).

We are not going to pursue the last parenthetical suggestions, for $R^{\sharp}$ itself will provide us with all the kinds of implication-relevant, roughly intuitionist, and material - that we need for our immediate investigations. Having sorted out the fact that $\rightarrow$ goes with $\circ$, the deduction theorem which is wanted is reflected evidently in the following provable R-equivalence:

○1. $A \circ B \rightarrow C \leftrightarrow A \rightarrow . B \rightarrow C$

For if $C$ is derivable from the fused premisses $A, B$, then and only then is $B \rightarrow C$ derivable from $A$.

Also important here will be the other principal R-properties of fusion, which are reflected in the following theses:

○2. $A \circ B \leftrightarrow B \circ A$

०3. $A \circ(B \circ C) \leftrightarrow(A \circ B) \circ C$

०4. $A \rightarrow A \circ A$

Since we also have $\wedge$ as a bunching connective, also licensing deductions from hypotheses in a certain sense (even though not directly tied to the deduction theorem for $\rightarrow$ ), we note for comparison the following theses.

$$
\begin{array}{ll}
\wedge 1 . & A \wedge B \rightarrow A, \quad A \wedge B \rightarrow B \\
\wedge 2 . & A \wedge B \leftrightarrow B \wedge A \\
\wedge 3 . & A \wedge(B \wedge C) \leftrightarrow(A \wedge B) \wedge C \\
\wedge 4 . & A \leftrightarrow A \wedge A
\end{array}
$$

Relating our two bunching connectives is the thesis

$$
\text { ^o. } A \wedge B \rightarrow A \circ B
$$

$\wedge \circ$ may seem to the reader to refute our thesis that $\wedge$ is looser than $\circ$. But it's just a matter of perspective; by the Directed Replacement Theorem, whatever we might have derived from the tight bunch $A \circ B$ we could have derived the loose bunch $A \wedge B$.

Having got thus far, let us, without further ado, begin to cast $R$, and 
afterwards $R^{\sharp}$, in such a form that the practical advantages of the natural deduction technique become available. To begin with, let us introduce

(i) $A \leq B$

as an abbreviation for

(ii) $\quad A \rightarrow B$ is a theorem of $\mathrm{R}$ (or other system)

Since it is useful to express every theorem of $\mathrm{R}$ in the relational form (i), and since not every theorem is of the preferred form $A \rightarrow B$ of (ii), it is useful to introduce into the language a sentential constant $\mathbf{t}$, subject to the following additional axiom scheme.

to. $\quad \mathbf{t} \circ A \leftrightarrow A$

The methods of [ENT] are easily adapted to show that $\mathbf{t}$ may be added conservatively to $R, R Q, R^{\sharp}$, etc. In fact, we shall have only passing use for a new constant $\mathbf{t}$ in $R^{\sharp}$, since we shall see that the formula $0=0$ will serve the role of this constant for all purposes that we are interested in here. But that needs to be shown, and meanwhile it is convenient to suppose that a primitive $\mathbf{t}$ is in the morphology (subject of course to to and all other axioms and rules). Strictly speaking, we should follow [ENT] and distinguish systems $\mathrm{R}^{\mathrm{t}}, \mathrm{RQ}^{\mathrm{t}}, \mathrm{R}^{\sharp^{\mathrm{t}}}$ with primitive $\mathbf{t}$ from systems $\mathrm{R}, \mathrm{RQ}, \mathrm{R}^{\sharp}$ without it; indeed, we should distinguish a language $\mathscr{L}^{\sharp}$ from our language $\mathscr{L}^{\sharp}$ that doesn't have primitive t. In fact, given our general conservative extension result in these circumstances, that degree of nice distinction is hardly necessary. What we shall do instead is to use primitive $\mathbf{t}$ when convenient, switching over to a $\mathbf{t}$ defined as $0=0$ once we have shown that to holds for this defined $\mathbf{t}$ in $R^{\sharp}$.

The utility of $\mathbf{t}$ springs immediately from the following theorem scheme.

$$
\mathbf{t} \rightarrow . \quad \mathbf{t} \rightarrow A \leftrightarrow A
$$

whence it is clear that we can express the R-theoremhood of any formula $B$, in the form

(iii) $\mathbf{t} \leq B$

Let us now get ourselves into a natural deduction frame of mind. We may Australasian Journal of Logic (18:5) 2021, Article no. 4 
think, in general, of $A$ as a formula compounded from subformulas $A_{1}, \ldots, A_{n}$ by $\circ, \wedge$, and perhaps other connectives. In particular, when the compounding connectives are just $\circ$ and $\wedge$, we are dealing with what, motivationally, is a bunch of premisses.

Note two facts about this compounding. First, the bunch depends not merely on $A_{1}, \ldots, A_{n}$ but on the character of the compounding. I.e., we may compound for a while by $\wedge$, compound again by $\circ$ (including some components already compounded by $\wedge$ ), compound again by $\wedge$, and so forth. Indeed, exactly this idea underlines the ingenious Dunn Gentzenization of $\mathrm{R}^{+}$that one finds in $[\mathrm{ENT}]$.

Second, however, the end result of this compounding is not necessarily that complex after all. For we may reduce it in various ways, using the principles $\circ 2-\circ 4, \wedge 1-\wedge 4$, and (in context) applications of the replacement theorems. In particular, any bunched formula $A$ is equivalent to one that one gets by commuting or associating o components, or by commuting, associating, or dropping idempotents among $\wedge$ components. So we can afford to be a little bit loose (as we shall be) in permuting and otherwise manipulating our bunched components. (We cannot, however, afford to be too loose, for only restricted distribution principles interrelate $\wedge$ and $\circ$.)

Being in a natural deduction frame of mind, however, we may now understand an assertion

(i) $A \leq B$

as a claim that $B$ is deducible from the premisses $A_{1}, \ldots, A_{n}$ bunched in $A$, given that they are bunched in exactly the $A$ way (or in some equivalent way). And we note in particular that whenever (i) holds, and $A$ is bunched under $\circ$ and $\wedge$ from $A_{1}, \ldots, A_{n}$, then the following also holds:

(iv) $\quad A_{1} \wedge \ldots \wedge A_{n} \leq B$

no matter how many iterations of the $A_{i}$ there are in the bunch $A$, or how intricate the bunching becomes. (For proof, use directed replacement and $\wedge \circ$ to eliminate all bunching occurrences of $\circ$ in favor of $\wedge$, and then rearrange and drop idempotents by $\wedge 2-\wedge 4$ ). Note moreover that we can, by $\wedge 1$, add "new" conjuncts $C_{j}$ indefinitely to the $A_{i}$ in (iv).)

That (i) can be traded in for (iv) is, in a sense, the classical residue of the relevant theory of deducibility. What it means systematically in $\mathrm{R}$ is that 
any deduction from hypotheses may be recorded in the form (iv). Often, in practice, concentrating on this classical residue of the relevant theory is a good thing; why should we bother with intricate and iterated compounding under both $\wedge$ and $\circ$ when we can reduce all our compounding to the form (iv)? Sometimes, however, such concentration is a bad thing. For the passage from (i) to (iv) is essentially an information-losing passage. Recall that compounding under $\circ$ required us to use all of the compounded components in a relevant way (as $R$ understands relevance), whereas compounding under $\wedge$ imposes no such requirement. In particular, when we trade in a o-compound for an $\wedge$-compound, we cannot make any use of the form of the deduction theorem embedded in 01 to "discharge" a hypothesis $A_{n}$, in order to assert a relevant conditional $A_{n} \rightarrow B$ under the remaining hypotheses.

So a relevant theory of natural deduction must make room for both ocompounding and $\wedge$-compounding of hypotheses. In fact, this has been at least implicit, and often explicit, in relevant logics from the start, as the careful reader of [ENT] will have ascertained. And it is a defect of the [ENT] approach to relevant natural deduction, which has at times confounded both friend and foe of the relevant enterprise in general, that its set-theoretical representation of $\circ$ via tagging devices biases this approach in favor of $\circ$ and against $\wedge$.

Again and again it has turned out to be the case that the relevant logics of $[\mathrm{ENT}]$ are more nearly classical, in more ways than one would have thought, than one would have supposed from some of the polemic which was introduced to motivate them. The new insights, to be sure, are the more precious; but old insights are everywhere incorporated, not least in the $\wedge$ vs. $\circ$ business that we have just been looking at. For it was, in fact, a bit of a tour de force for $[\mathrm{ENT}]$ to have concentrated on representing o set-theoretically, via its natural deduction techniques, when it is the more familiar connective $\wedge$ (and $\vee$, for that matter), with which we might have expected to have associated straightforward set-theoretical operations.

Now what happens, to put it roughly, is the following. Semantical techniques (e.g., those of $[\mathrm{SEI}]$ ) do associate $\cap, \cup$ respectively with $\vee, \wedge$. But natural deduction techniques as in $[\mathrm{ENT}]$, given a semantical cast by Urquhart in [SRL] associate $\cup$ rather with $\circ$. One wants to associate $\cap$ with $\wedge$ nevertheless, as Urquhart in effect does. But this leaves $\vee$ out in the cold, so to speak; it yearns after its old friend $\cup$, but $\cup$ is already going steady with $\circ$. Climbing a set-theoretical level, this can be partially set to rights; $\cup$ becomes a bigamist, living with $\circ$ downstairs and with $\vee$ upstairs.

Australasian Journal of Logic (18:5) 2021, Article no. 4 
But bigamy, though exciting, is rarely satisfactory. $\cup$, by dividing its time between $\circ$ and $\vee$, treats neither of them really well in terms of fundamental relevant motivation. In particular, the duality between $\wedge$ and $\vee$ is upset; since $\sim$, as the negation connective of the systems of [ENT], rests heavily on this duality, its theory becomes maximally mutilated. And so one hears proposals, finally, to change the relevant theory of disjunction, and to throw out the relevant theory of negation for some more familiar sort (i.e., roughly intuitionist or semantically classical) on what are held to be fundamental relevant grounds.

I shall not identify the authors of these proposals, since one wishes to give them the opportunity to think better of them. But it is, at any rate, nonsense to think that any fundamental relevant motivation is here involved. In particular, no topic in the relevant logics has been more often or more thoroughly motivated, from a large number of points of view, than their theory of negation. If something has got to go, it is scarcely the theory of $\sim$. Nor, for that matter, is it the theory of $\wedge$ and $\vee$. Left for possible revision are the theory of $\circ$ (which is anyway system-dependent, and which is inextricably linked via deduction-theorem considerations with the theory of $\rightarrow$ ) and of relevant implication itself.

Now, personally, I do not see the theory of $\circ$ as in any sense univocally motivated. So I do not attach any particular importance to the AndersonBelnap-Urquhart way of representing that theory. And I find it positively perverse to suppose that this representation, designed to make formal sense of $\rightarrow$ (and, by extension, of $\circ$ ), as as a test of its adequacy its extendibility to make the same sort of sense of $\wedge, \vee, \sim$. Once $\circ$ has been tied to $\cup$, in the $[\mathrm{ENT}]$-style marriage of formal convenience, all bets are off as to what $\vee$, or even $\wedge$, should be tied to.

Since I have not wished to go into formal details in the last few paragraphs - essentially, they are spelled out in [IDP] - I must acknowledge a certain degree of historical misrepresentation. Both the Anderson-Belnap analysis of relevant natural deduction and the Urquhart semantical approach based on it have been a tremendous boon to the relevant enterprise in general. Nor did anyone associated with either project, so far as I know, think of himself as using $\cup$ to do the work of $\circ$. But that, I think, is what both projects amount to, accounting alike for their successes and their limitations. And, both systematically and semantically, I think it better to let $\circ$ do the work of $\circ$; for it is o that has systematic significance, which we might as well attack directly as to take as set-theoretical code for something else, and 
which, decoded, is going to come to the same thing anyway. And that frees $\wedge$ to do its own work, which, if we choose, we can more naturally take as set-theoretical work, without fear that we are thereby lousing up the code.

These, then, are further reasons why we have cut loose from the [ENT] techniques and are concentrating on letting $\mathrm{R}$ speak for itself. In the end, however, we shall have something that looks very much like the [ENT] technique anyway, especially for $\mathrm{R}$ (though, mutatis mutandis, for other systems as well), while retaining at all points a firm systematic base in $\mathrm{R}$ itself.

Our basic "natural deduction" statements will remain firmly of the form

(i) $A \leq B$

But we shall dress up statements (i) so that they look like proper natural deduction statements, by converting $A$ itself into a "tag" for the formula $B$. And, as a tag, $A$ will work (near enough) like the sets of relevance numerals which function as tags in [ENT]. But since, in the end, $A$ remains firmly a (single) formula, we can replace it in accordance with the directed replacement theorem, giving us a freedom of tag manipulation not available when tags are playing a mere auxiliary role.

To strengthen the useful psychological impression that we are dealing straightforwardly with tagged formulas $B$, and to avoid the wasteful and perhaps confusing need to write out formulas $A$ that appear in (i) in full, we shall use Greek letters ' $\alpha$,' ' $\beta$,' etc., as an alternative notation involves no change. Practically, it has an abbreviatory character. Thus, in writing out actual "natural deductions," we make use of statements of the form

$$
\text { (v) } \alpha=A
$$

Statements (v) are to be understood as asserting that $\alpha$ is exactly the same formula as $A$. I.e., the identity in question is not any kind of provable equivalence, but is actual identity of the formulas being referred to. Naturally context will prevent any confusion of the (metatheoretic) statements (v) with our use of the formal identity $=$ of the system $R^{\sharp}$.

Practically, (v) just records an abbreviation. Psychologically, however, we may regard a statement (v) as having introduced $A$ "by hypothesis." $\alpha$, on this understanding, is just a tag, which will serve the purpose in a "natural deduction" of helping us to keep track of which "conclusions" depend upon this "hypothesis" A. Really, however, this is just notational manipulation. 
For, on our abstract understanding of formal systems generally, formulas themselves are taken as abstract objects, and it is a matter of complete indifference whether ' $\alpha$ ' or ' $A$ ' or ' $0=0$ ' is being used as a piece of notation in English to refer to some such object. As pieces of notation, at any rate, we may accordingly interchange the left and the right sides of $(\mathrm{v})$ whenever it is convenient, in any subsequent statement (subject, of course, to the proviso that we do not introduce the same Greek letter in the course of a derivation to name two distinct formulas).

Statements (v), then, will play here the role of assumption procedures in customary natural deduction systems. Note that, in view of the theorem scheme $A \rightarrow A$ of $\mathrm{R}$, we may pass immediately on what has been said from a statement $(\mathrm{v})$ to

$$
\text { (vi) } \quad \alpha \leq A
$$

We shall ordinarily leave such steps tacit, citing merely a step (v) to justify whatever use we may make rather of (vi).

Assumption procedures, it was held above, are one of the three main ingredients of natural deduction systems. I turn now to the third of these ingredients, namely discharge procedures. Or rather to the most salient discharge procedure, namely a rule $\rightarrow I$ which allows us to derive a conclusion $A \rightarrow B$ by "assuming" $A$ and "showing" $B$.

Suppose, then, that we have reached a statement

$$
\text { (vii) } \quad \Gamma \circ A \leq B
$$

where $\Gamma$ is some formula (perhaps in compounded Greek disguise and perhaps just, notationally, in solid, stolid Roman). In view of o1, we may conclude immediately (by the "relevant" "deduction theorem")

$$
\text { (viii) } \quad \Gamma \leq A \rightarrow B
$$

and we may append the notation $\rightarrow I$ to justify the move.

More likely, not only $\Gamma$ but also $A$ will be in Greek disguise. I.e., we shall have reached, from a "hypothesis" step (v), a "conclusion"

$$
\text { (ix) } \quad \Gamma \circ \alpha \leq B
$$

Then from (v) and (ix) together, we may conclude, by a "two premiss rule" Australasian Journal of Logic (18:5) 2021, Article no. 4 
$\rightarrow I$, once more the statement (viii).

Now note that, in this latter incarnation, we have indeed reached a familiar (relevant) natural deduction pattern. We assumed $A$, in step (v), with $\operatorname{tag} \alpha$. From this assumption, we got in step (ix) the formula $B$, "tagged" with $\alpha$ to indicate dependence on the hypothesis (v), where $\alpha$ is compounded in a tag $\Gamma \circ \alpha$ such that $\Gamma$ keeps track of the "other assumptions" used in deriving $B$. So we may discharge the hypothesis $A$, drop the tag $\alpha$ that kept track of that hypothesis, and conclude $A \rightarrow B$, tagged with the "other assumptions" $\Gamma$ on which its derivation thus far depends.

Next let us turn to working rules. They constitute, we said above, the heart of the natural deduction procedure. Moreover, they may be linked formally to the directed replacement theorem, which takes the necessary heed of those rules which may be applied invariantly under assumptions. But this link suffers two possible complications. First, we must distinguish between 1-premiss rules and multi-premiss rules. Second, we must attend to those complications which are occasioned by the proper care and feeding of quantifiers.

Let us begin where we are clearest, by dealing with simple 1-premiss rules into which quantificational complexities do not enter. $\wedge E$ above is an example. Such rules are normally straight replacement, involving (usually) nothing more complicated than transitivity of the provable implication $\leq$. Psychologically, however, we may look upon such rules as permitting some manipulation on a tagged formula $B$. Thus, viewed generally, whenever we are given

\section{(x) $\quad \Gamma \leq B$}

when we have established as a theorem of $R$ ( or $R Q$ or $R^{\sharp}$ ) that

$$
\text { (xi) } B \leq C
$$

we may conclude by the "one premiss rule" (xi) from the "tagged formula" (x) the following:

(xii) $\quad \Gamma \leq C$

Psychologically, (xii) is also a "tagged formula," with $C$ bearing the same "tag" $\Gamma$ as does its "premiss" $B$ in (x).

Australasian Journal of Logic (18:5) 2021, Article no. 4 
In order to give the reader a good stock of these simple "one premiss rules," it suffices to write down a number of the provable implications and equivalences of $\mathrm{R}$ and of $\mathrm{RQ}$. For this purpose, as we use $A \leq B$ to indicate the R-provability of $A \rightarrow B$, let us use $A \simeq B$ to indicate the R-provability of $A \leftrightarrow B$. Evidently, when a statement (xi) is of the form $B \simeq C$, it licenses an "inference" (xi) from (xii) to (x) as well as from (x) to (xii). Moreover, we shall attach "natural deduction" style names to many of these provable implications, to encourage the reader further to identify them psychologically with inferences from (x) to (xii). I.e., we shall refer to theses $\wedge 1$ above as $\wedge E$, to foster the illusion that these constitute a rule for the elimination of conjunctions in the course of a deduction. Similarly, we shall refer to $\wedge 2$ as $C \wedge, \wedge 3$ as $A \wedge$, and $\wedge 4$ as $I D \wedge$, to remind the reader that inferences depending on the commutativity, associativity, or idempotence of $\wedge$ are respectively permitted. Decoding other mnemonics is left to the reader's good sense. He may begin by finding grounds for our subsequent habit of referring to $\circ 2$ as $C \circ, \circ 3$ as $A \circ$, and to the square-increasing principle $\circ 4$ which says that $A \leq A \circ A$ as $S Q \circ$. He already knows, of course, why we refer to the principle $\circ 1$, sometimes in Greek disguise, as $\rightarrow I$. He may practice his decoding further on the following:

$$
\begin{array}{lll}
\vee I . & A & \leq A \vee B \\
& B & \leq A \vee B \\
\sim \sim E I . & \sim \sim A \simeq A & \\
D \wedge \vee . & A \wedge(B \vee C) & \simeq A \wedge B \vee A \wedge C \\
D \vee \wedge . & A \vee(B \wedge C) & \simeq(A \vee B) \wedge(A \vee C) \\
C \vee . & A \vee B & \simeq B \vee A \\
A \vee . & A \vee(B \vee C) & \simeq(A \vee B) \vee C \\
I D \vee . & A \vee A & \simeq A \\
D \sim \wedge . & \sim(A \wedge B) & \simeq \sim A \vee \sim B \\
D \sim \vee . & \sim(A \vee B) & \simeq \sim A \wedge \sim B \\
D \rightarrow \wedge . & A \rightarrow B \wedge C & \simeq(A \rightarrow B) \wedge(A \rightarrow C) \\
D \vee \rightarrow . & A \vee B \rightarrow C & \simeq(A \rightarrow C) \wedge(B \rightarrow C) \\
S F \rightarrow . & A \rightarrow B & \leq B \rightarrow C \rightarrow A \rightarrow C \\
P F \rightarrow . & B \rightarrow C & \leq A \rightarrow B \rightarrow . A \rightarrow C \\
M P \rightarrow . & A & \leq A \rightarrow B \rightarrow B \\
M \rightarrow \wedge . & A \wedge(A \rightarrow B) & \leq B
\end{array}
$$

Australasian Journal of Logic (18:5) 2021, Article no. 4 


$$
\begin{aligned}
& \text { RDA. } A \rightarrow \sim A \leq \sim A \\
& \text { RDB. } A \rightarrow B \wedge \sim B \leq \sim A \\
& \text { RDC. } A \rightarrow B \circ \sim B \leq \sim A \\
& D \rightarrow \sim . \quad A \rightarrow \sim B \quad \leq B \rightarrow \sim A \\
& D \rightarrow \rightarrow . \quad A \rightarrow B \rightarrow C \leq A \rightarrow B \rightarrow . A \rightarrow C \\
& W \rightarrow . \quad A \rightarrow . A \rightarrow B \leq A \rightarrow B \\
& D \circ \wedge . \quad A \circ B \wedge C \leq(A \circ B) \wedge(A \circ C) \\
& R \wedge \circ . \quad A \wedge B \quad \leq A \circ B \\
& R \rightarrow \supset . \quad A \rightarrow B \quad \leq A \supset B
\end{aligned}
$$

In addition, we retain for the $\mathbf{t}$-systems the principles $\mathbf{t} \circ, \mathbf{t} \rightarrow$. These lead quickly to

$$
\text { t } I D . \quad \mathbf{t} \leq A \rightarrow A
$$

and in general to, wherever $B$ is a theorem of the system in question

$$
\mathbf{t} T R . \quad \mathbf{t} \leq B
$$

Moreover, defining $\mathbf{f}$ generally as $\sim \mathbf{t}$, we have also

$$
\sim E I \mathbf{f} . \quad A \rightarrow \mathbf{f} \simeq \sim A
$$

$\sim$ EIf functions, in Curry's terminology, as a "quasi-definition" of $\sim$ here in terms of $\rightarrow$ and $\mathbf{f}$. Moreover, from left to right it may be assimilated to the "reductio" principles $R D A-R D C$.

This is but a sampling of useful "natural deduction rules," under our stated policy, for $R, R Q, R^{\sharp}$. To prepare ourselves for quantificational complications, note that the following "rules" hold without restriction.

$$
\begin{aligned}
& \forall E . \quad \forall X A[X] \leq A[T] \\
& \exists I . \quad A[T] \leq \exists X A[X] \\
& D \forall \wedge . \quad \forall X(A \wedge B) \simeq \forall X A \wedge \forall X B \\
& D \exists \vee . \quad \exists X(A \vee B) \simeq \exists X A \vee \exists X B \\
& D \circ \forall . \quad \forall X A \circ \forall X B \leq \forall X(A \circ B) \\
& D \forall \rightarrow . \quad \forall X(A \rightarrow B) \leq \forall X A \rightarrow \forall X B \\
& R \rightarrow \exists . \quad \forall X(A \rightarrow B) \leq \exists X A \rightarrow \exists X B
\end{aligned}
$$

Australasian Journal of Logic (18:5) 2021, Article no. 4 
$\begin{array}{lll}Q \forall \exists . & \sim \forall X B & \simeq \exists X \sim B \\ Q \exists \forall . & \sim \exists X B & \simeq \forall X \sim B\end{array}$

In $\forall E, \exists I, T$ is any term properly substituted for $X$ in $A[X]$.

Let us now think about the quantifier problems, while still dwelling on 1-premiss rules. These problems arise, principally, because the question whether the application of a rule is invariant under hypotheses is sensitive to questions of freedom and bondage. (In certain respects, this means that it is a notational question, since there is a certain equivocation in allowing the same variable $X$ to function both as a free variable and as a bound variable. It is convenient to permit such equivocation here, and so we do.) Accordingly, we should note the following principles dealing with vacuous quantification and confinement in $\mathrm{RQ}$ and $\mathrm{R}^{\sharp}$. Assume, for the following theses, that $X$ is not free in $A$.

$$
\begin{aligned}
& V Q \forall . \quad \forall X A \simeq A \\
& V Q \exists . \quad \exists X A \simeq A \\
& C F \wedge . \quad \forall X(A \wedge B) \simeq A \wedge \forall X B \\
& \exists X(A \wedge B) \simeq A \wedge \exists X B \\
& C F \vee . \quad \forall X(A \vee B) \simeq A \vee \forall X B \\
& \exists X(A \vee B) \simeq A \vee \exists X B \\
& \text { CF。. } A \circ \forall X B \leq \forall X(A \circ B) \\
& A \circ \exists X B \simeq \exists X(A \circ B) \\
& C F \rightarrow . \forall X(A \rightarrow B) \simeq A \rightarrow \forall X B \\
& \forall X(B \rightarrow A) \simeq \exists X B \rightarrow A \\
& \exists X(A \rightarrow B) \leq A \rightarrow \exists X B \\
& \exists X(B \rightarrow A) \leq \forall X B \rightarrow A
\end{aligned}
$$

Bound variables, too, may be rewritten at will. I.e., letting $A, A^{\prime}$ be alphabetic variants, we have

$$
R B V . A \simeq A^{\prime}
$$

Thus far, none of our 1-premiss "quantificational rules" are sensitive to questions of invariance under assumptions. Thus, e.g., we are always free, given a "tagged formula"

Australasian Journal of Logic (18:5) 2021, Article no. 4 
(xiii) $\quad \Gamma \leq \forall X A[X]$

to conclude without restriction

(xiv) $\quad \Gamma \leq A[T]$

for any term $T$ by $\forall E$. For, from the systematic viewpoint of $\mathrm{R}$ or $\mathrm{R}^{\sharp}$, this is just another application of the replacement theorem. But, psychologically, we apply a "rule" $\forall E$.

What, however, of the converse inference? I.e., given

(xv) $\quad \Gamma \leq A[X]$

under what circumstances can we conclude (xiii)?

Again, we make use of our psychological interpretation of (xv) as a tagged formula $A[X]$, with tag $\Gamma$ keeping track of dependence relations, combined with our actual interpretation of $(\mathrm{xv})$ as stating a certain provable implication. Psychologically, the hypotheses which $\Gamma$ tracks cannot contain free $X$, if we are to generalize legitimately on $A[X]$ to get $\forall X A[X]$. For a rule of universal generalization is not invariant under hypotheses that contain free $X$.

Actually, however, $\Gamma$ is not merely a tag keeping track of hypotheses; it is the hypotheses, perhaps in Greek disguise as a result of steps (v). What (xv) says is that $\Gamma \rightarrow A[X]$ is itself a theorem of the relevant system in question. But universal generalization on theorems permits us to infer $\forall X(\Gamma \rightarrow A[X])$ as a further theorem. And then, if $X$ is not free in $\Gamma$, so also is $\Gamma \rightarrow \forall X A[X]$ a theorem, by $C F \rightarrow$. I.e., (xiii) holds.

So, in short, the inference from (xv) to (xiii) is O.K. just in case $X$ is not free in the "tag" $\Gamma$ on the "tagged formula" $A[X]$. And this restriction makes perfectly good sense, not only actually but psychologically. In practice, where a tag is generally in Greek, it suffices to check through Greek components of the tag (e.g., $\alpha$ ) introduced by a "hypothesis" (e.g., $\alpha=A$ ) by checking mechanically whether $X$ occurs free in the formulas (e.g., $A$ ) which these Greek components abbreviate. (Alternatively, we might keep a running record of the free variables in our hypotheses by writing our tags in the form $\alpha\left(X_{1}, \ldots, X_{n}\right)$, where $X_{1}, \ldots, X_{n}$ are the variables free in the formula $A$ with which $\alpha$ is associated by a "hypothesis" step (v). Just checking back, however, tends to be simpler.)

Australasian Journal of Logic (18:5) 2021, Article no. 4 
Let us make a list of some useful "natural deduction" rules, in addition to those immediately motivated by directed replacement above, that depend on the ideas just presented. We assume, to state these rules, that $X$ is free neither in the "tag" $\Gamma$ nor in the formula $A$.

$$
\begin{array}{llll}
\forall I . & \Gamma \leq B & \Rightarrow & \Gamma \leq \forall X B \\
C \forall I . & \Gamma \leq A \vee B \quad & \Rightarrow & \Gamma \leq A \vee \forall X B \\
C \forall \forall . & \Gamma \leq B \rightarrow C \Rightarrow & \Rightarrow \leq \forall X B \rightarrow \forall X C \\
C \exists \exists . & \Gamma \leq B \rightarrow C \Rightarrow & \Rightarrow & \Gamma \leq \exists X B \rightarrow \exists X C \\
R \forall I . & \Gamma \leq A \rightarrow B \Rightarrow & \Rightarrow & \Gamma \leq A \rightarrow \forall X B \\
R \exists E . & \Gamma \leq B \rightarrow A \Rightarrow & \Rightarrow & \Gamma \leq \exists X \rightarrow A
\end{array}
$$

Note that, in contrast to our previous "rules" like $\wedge E, \forall E$, which were theorems disguised as rules, the six rules above are genuine admissible rules of $R Q, R^{\sharp}$, and other theories. I.e., each of them states that from the theoremhood of a formula of a certain form, indicated schematically on the left hand side of the main $\Rightarrow$, we may conclude that a related formula is also a theorem, indicated schematically on the right.

We have now provided a decent inventory of 1-premiss rules. Note that this inventory is intended for use; the reader may remember what he pleases from it (most of which, no doubt, will already be familiar to him from other contexts). From a conceptual viewpoint, nothing in the inventory is necessary; $\mathrm{R}^{\sharp}$ was already completely characterized by its axioms and rules above, and those principles alone suffice to derive all its theorems. We are not, after all, justifying $\mathrm{R}$ here, but instructing the reader how best to get on in it. And, in fact, natural deduction systems are often caught between their use to "justify" certain insights and their practical use in developing the consequence of these insights. But this kind of concern for motivation often interferes with practicality; thus we are treated to distinctions between "unabbreviated derivations" in natural deduction systems, which rest only on the rules which are supposed to reflect the insights, and "abbreviated derivations," which yield short-cut procedures on how to get on. My view is that, except for very limited and specific purposes, even introducing the notion of an "unabbreviated derivation" in a natural deduction system is a waste of time. (Such purposes, e.g., might lie in proving "cut" or "normalization" theorems, which have various kinds of metatheoretic import.) Generally, however, we expect makers of natural deduction systems to have a proof up 
the sleeve that their systems are sound and complete relative to something else - semantic, syntactic, or both - and it is an insult to our collective intelligence to suppose that the characterization of "unabbreviated derivation" goes much further than that enough principles have been stated to justify the proof up the sleeve. So there is little point, I think, in having the proof up the sleeve at all. Let it be on the table, and let every derivation be an "abbreviated derivation," to the limit of what the deriver knows about the system in question.

To push this point just a bit further, there has been, in recent years, a certain trend away from the inculcation of systematic familiarity with formal systems. One rushes off to prove metatheorems about the systems instead. In part, I agree with this trend; we need to see theories whole, and not to get bogged down in their devices. But, in another part, I think it unfortunate. While the metatheorems, I agree, are the important theorems, by and large, we are not too likely to come upon them if we stipulate our systems principally with the production of old metatheorems in mind. Nor are we too likely to be able to apply our systems to real-life questions without considerable working knowledge of how to get about in them. And thus, e.g., I agree with Scott's remarks in [AML], to the effect that too much absorption in projects like proving completeness is logically unhealthy. If we know that a system is complete, there is little benefit in pretending to discover it (or, even worse, pretending not to know it). Let us hasten, rather, to use what we know, for the point of inquiry is to find out what we don't know.

We used directed replacement, mainly, to get our 1-premiss rules. Given a "tagged formula" $\Gamma \leq A$, and a theorem $A \leq B$ masquerading as a rule, we derive by directed replacement the "tagged formula" $\Gamma \leq B$. What happens really is that we are replacing a positively charged $A$ with a positively charged $B$, in accordance with directed replacement. But what happens psychologically is that we are just inferring $B$ from $A$, in accordance with the "rule," and keeping the tag constant to show that dependence on the hypotheses hasn't changed.

Directed replacement, however, works just as well in reverse, a fact which we put to some slight use above. In reverse, it is a principle of "tag replacement." E.g., suppose that we are given

$$
\text { (xvi) } \quad \Delta \leq B
$$

while having otherwise established (perhaps on elementary properties of sim- 
ple connectives like $\circ, \wedge, \vee$ )

$$
\text { (xvii) } \quad \Gamma \leq \Delta
$$

Then by directed replacement of the negatively charged $\Delta$ in (xvi), we are entitled to conclude by (xvii)

$$
\text { (xviii) } \quad \Gamma \leq B
$$

Really, this is just another passage by transitivity of $\leq$. Psychologically, however, moving from (xvi) to (xviii) is just changing the tag on the "tagged formula" $B$. Since tags are used to keep track of hypotheses - in practice, of bunched hypotheses - tag-changing means that $B$ would still have been derivable under certain permutations of hypothesis-bunching, or even perhaps under some significant change in the hypotheses themselves.

Since our favored bunching connectives $\wedge$, $\circ$ are awash with nice properties (commutativity, associativity, etc.) in $\mathrm{R}$, we can, as essentially noted above, re-tag quite liberally. (In fact, we shall in practice refrain from retagging, trusting the reader to supply an admissible re-arrangement by commutativity, etc., when he sees one.) For weaker relevant logics, to the contrary, close attention to "re-tagging" principles is in order. For, on the line of [ALI] (and of Maksimova's independent work to the same effect), it is exactly with respect to the re-tagging principles that are admitted that relevant logics differ. (Cf. [NAR] for further motivation on this point.) Indeed, careful attention on this point will lead to exactly the kinds of result - cut theorems and the like - which are eschewed here. For, as Pascal rightly noted, to have arranged things differently is not necessarily to have said the same thing. Or, we add, to have drawn the same conclusions.

In $\mathrm{R}$, where tag manipulation is normally so simple that explicit mention of the manipulative principle is rarely required, there remains one point worth noting. Suppose that we have derived, say

(xix) $\quad \alpha \circ \alpha \leq B$

Then we are entitled, by $S Q \circ$ and a negative replacement in (xix), to conclude

$$
\text { (xx) } \quad \alpha \leq B
$$

Note, however, that this does not work in reverse; o is only semi-idempotent, Australasian Journal of Logic (18:5) 2021, Article no. 4 
not idempotent, and so we cannot in general conclude (xix) when we are given $(\mathrm{xx})$.

But we can, however, use a general principle, reflected in the passage from (xix) to $(\mathrm{xx})$, for tag manipulation of tags compounded under $\circ$. First, by associativity and commutativity of o, we may rearrange at will. Then, as in passing from (xix) to (xx), we may drop iterated tags whenever we please. (For a specific purpose, however, it is not necessarily wise to do so; for ( $\mathrm{xx}$ ) is a weaker statement than (xix), and it is possible that the stronger statement is in fact required.)

That we can manipulate tags, as we have seen, is the result of having a suitable replacement theorem, together with the actual provable implications and equivalences that license a particular replacement. Mainly, we used above only a very weak form of replacement-namely, that $\leq$ is transitive. In tag manipulation, however, the strong form comes in handy. For often, we might wish to replace a tag which occurs, so to speak, as a sub-tag of a bigger tag. For example, given

$$
\text { (xxi) } \quad \Gamma \circ(\alpha \circ \alpha) \circ \Delta \leq B
$$

we might wish to conclude by $S Q \circ$

$$
\text { (xxii) } \quad \Gamma \circ \alpha \circ \Delta \leq B
$$

And this is most easily seen as a replacement within a tag, not a replacement of a whole tag. Note, incidentally, that taking tags as bunches of hypotheses (bunched by $\circ, \wedge$, and even by $\vee$ if we like) is nicely set up for directed replacement in this context. For the connectives in question are positive with respect to all their arguments, which means that all tags bunched by them occur negatively as sub-tags of $\Gamma$ in a statement of the form $\Gamma \leq B$. So, with these bunching connectives, we can make, essentially, the same replacements within tags as we could have made on tags taken as wholes to be replaced. From this perspective, the passage from (xxi) to (xxii) is the same passage as that from (xix) to (xx). And having this form of replacement is particularly useful (e.g., the Dunn proof of "cut" in his [ENT] Gentzenization depends on it), for it allows the replacement of sub-tags deeply nested (say by overlapping and iterated applications of $\wedge, \circ$ ) within the tags in which the replacements are made.

Such principles of "tag replacement" are indeed key features of the natural

Australasian Journal of Logic (18:5) 2021, Article no. 4 
deductions that one finds in [ENT]. But this is largely obscured by the fact that fusion is "hidden" in the [ENT] system in the guise of set union, as noted above, so that such properties of fusion as commutativity and associativity are built in. (More interesting is the way in which an idempotent set union comes to "represent" a fusion which is only semi-idempotent.)

Using the [ENT] technique, I fear, is like learning Japanese. The first lessons are delightful, but their simplicity is illusory. For one has to be at home with the systematic representation of the language, via its own ideograms, to be at home with the language itself; simplified Western spelling helps with lesson 1, but it becomes a positive obstacle by lesson 50 . And it is mere cultural chauvinism for the learner to suppose that the "intelligibility" of a new language is in any way dependent on its successful reduction to his old alphabet. It may be so reducible, perhaps, but such reduction may conceal more central features than it reveals.

Just so, the [ENT] success in using $\cup$ to do the work of $\circ$ is largely illusory. For, whatever our principles of tag replacement, they need to be specifically and systematically justified. Instead, especially for the system $T$ of ticket entailment, [ENT] has to find ways to inhibit the tag replacement principles, using $\cup$, that it has assumed. This produces over-dependence on particular features of the Fitch-style subproof formulation. And, critically, the $[\mathrm{ENT}]$ procedures (since, conceptually, they fit o) fail to behave smoothly with respect to the truth-functions. There is no particular reason why they should.

In fact, by letting $\circ$, and also $\wedge$ and $\vee$, do their own work, instead of resorting to artificial auxiliary devices, there is not even very much to remember. For it is, after all, no more difficult to keep in mind the properties of $\wedge$ and $\vee$ than to keep in mind those of $\cap$ and $\cup$. They are, near enough, the same properties. And the properties of $\circ$ are those which multiplication has, as a first approximation, on natural numbers; keeping them in mind isn't that hard, either. And, importantly, these properties - unlike the [ENT] representation of $\circ$ via $U$ - have direct systematic significance. We know something about R - and not just about scorekeeping for R - when we know what they are. So, again, there is no point in appealing to auxiliary devices to do for $\mathrm{R}$ what we can already do with the devices which the system itself makes available. To the contrary (as anyone who has worked in detail with the [ENT] systems is aware), we often complicate arguments when we are compelled to put them in the special forms to which the auxiliary devices apply.

Simple tag manipulations are henceforth taken for granted, as stated

Australasian Journal of Logic (18:5) 2021, Article no. 4 
above. Complex ones will draw a note. In either case, the reader is invited to observe that it is the same fundamental directed replacement theorem which is doing the real work.

Thus far, we have concentrated on 1-premiss rules, in our "natural deduction" format. But we must think about multi-premiss rules as well. To illumine several points, let us think about extending our conjunction introduction rule $\wedge I$ to the natural deduction situation. What is it for such a rule to be invariant under assumptions?

First, suppose that we are given "tagged formulas"

$$
\begin{array}{ll}
\text { (xxiii) } & \Gamma \leq A \\
\text { (xxiv) } & \Gamma \leq B
\end{array}
$$

from which we wish to conclude

$$
\text { (xxv) } \quad \Gamma \leq A \wedge B
$$

In fact, the passage from (xxiii) and (xxiv) to (xxv) takes place immediately on R-valid principles. (For, keeping in mind what our "tagged formulas" mean, we may get (xxv) immediately from the primitive rule $\wedge I$ of $\mathrm{R}, A 5$, and $\rightarrow E$.)

The form of the $\wedge I$ rule just set out is, in principle, that of [ENT]. But it lacks generality. For suppose that we are given, together with (xxiii), not (xxiv) but

$$
\left(\text { xxiv }^{\prime}\right) \quad \Delta \leq B
$$

I.e., suppose that the "tags" on the 2 "tagged formulas" that we wish to conjoin are not the same.

The [ENT] reply is that $\wedge I$ is then blocked. So if $A, B$, each derived in the course of a natural deduction derivation, bear distinct tags (indicating roughly that they depend on distinct bunches of hypotheses), we can infer nothing by $\wedge I$, however minimally these bunches may differ. (Actually, the tag identity criteria in [ENT] are stricter yet; in order to square the idempotence of $\cup$ with the semi-idempotence of $\circ$, [ENT] may attach different tags to the same assumption formula.) In short, applying $\wedge I$ invariantly under assumptions is taken in [ENT] in a ferociously strong sense.

And this leads, as Prawitz, Urquhart, and Pottinger may be taken to have

Australasian Journal of Logic (18:5) 2021, Article no. 4 
seen, to practical difficulties in actually deducing things in R. Accordingly, given (xxiii) and $\left(\mathrm{xxiv}^{\prime}\right)$, and when tags are sets in the [ENT] fashion, it is proposed that the following conclusion be drawn by $\wedge I$

\section{(?) $\quad \Gamma \cap \Delta \leq A \wedge B$}

(I am assimilating, just for the moment, these ideas to our notation.)

We already commented roughly above on the idea behind (?). Tags in $[\mathrm{ENT}]$ are sets, and so when tags are compounded in [ENT] (on application of some multi-premiss rule which compounds tagged formulas), what could be more natural than some accompanying set-theoretical compounding of tags? And, compounding tags under $\cup$ having been reserved for other purposes, why not compound them under $\cap$ when applying $\wedge I$ ?

We have told the reader why not above. This kind of ad hoc manipulation of the scoreboard mutilates central motivating considerations for the truthfunctions. But (?) is right in principle, however questionable it becomes in practice when linked to certain specific directions for keeping set-theoretical score. For the key fact is that we don't need a set-theoretical scoreboard; $\wedge$ is clear enough, and we are not better informed as to its properties when $\cap$ flashes up on the scoreboard instead. So the correct conclusion to draw from (xxiii) and $\left(\mathrm{xxiv}^{\prime}\right)$, reverting to our notation and our system, is just

$$
\left(\mathrm{xxv}^{\prime}\right) \quad \Gamma \wedge \Delta \leq A \wedge B
$$

I.e., for a correct and generally applicable natural deduction rule $\wedge I$, we simply compound the hypotheses (disguised as tags) in just the way that we compound the conclusion. When $\Gamma$ and $\Delta$ are the same, this leads immediately, by idempotence of $\wedge$, to our old conclusion (xxv). But we are no longer paralyzed in the presence of formulas whose tags differ, nor are motivational points disrupted in making the most of the valid core behind (?).

A bit more comment is in order on the tagging questions, for [ENT], that (?) raises. These questions are linked semantically to the analysis of relevant logics set out by Urquhart in [SRL] and other papers, while they are linked also to the Prawitz sketch of relevant natural deduction to be found in [ND], which underlies in turn the Pottinger analyses to be found in [PRL].

So, despite the short motivational shrift which I gave it above, there would seem to be an impressive and independent case to modify [ENT] in a way which leads to the (?) form of $\wedge I$. Perhaps so; given certain kinds of formal 
evidence, I might be persuaded in that direction myself; and it is anyway worth pursuing. But, at present, I have to hold that the direction rests on a misconception, which is signalled in the sort of motivational breakdown noted above. But the signal is not the misconception. What I think has happened rather is that the [ENT] scoreboard has been taken too seriously, and that, in consequence, the [ENT] systems, with their multiple motivation, have not been taken seriously enough. Indeed, it is with a certain admirable audacity that Anderson and Belnap, in [ENT], shape scoreboard to system, and are willing to bear certain roughnesses in scorekeeping for the sake of smoothness of overall system. And here too it should be noted, as Urquhart himself points out, that the apparent coincidence between his semantical approach to relevant logics and the natural deduction techniques of [ENT] isn't so coincidental, since the former was shaped out of reflection on the latter. This was, I think, a brilliant imaginative achievement, and a very fruitful one in the light of subsequent research. But it is also a seductive achievement (as projects of extensional reduction often turn out to be, including some that have been offered by Routley and me, a point that we explore in [ER]), in that the classical set-theoretical reduction tends to command an ontological respect which it does not deserve, except as a device for imaginative stimulation, and that one is tempted thereby away from an exploration of systematic insights in their own systematic contexts. In one sense, I have never pretended to be anything but ad hoc here (for I hold that systems cooperate in their own motivation, and look with suspicion on all claims that overlook such cooperation, a suspicion that is redoubled when the systems in question are systems of "natural deduction"). But it is a forthright and democratic ad hocness; a system, in speaking for itself, must provide its own links with the informal motivation that it claims and seeks to shape, and its claims are not advanced by formal friends in fashionably high places.

So (?), as the valid conclusion of a rule that [ENT] should have had but missed, is still out; on its own grounds, and given its own motivation, [ENT] wants no part of that conclusion. Or, rather, it wants its valid core, which it gets straightforwardly on its own ground with $\left(\mathrm{xxv}^{\prime}\right)$.

Having been given a clue from $\wedge I$, let us now think about many-premiss rules in general. From a psychological viewpoint, as we can now see, the crucial new point is "tag" compounding. Given premisses with (in general) distinct tags, how shall these tags be compounded in order to find the "right" tag with which the conclusion is to be tagged? Moreover, since compound tags on these intuitions reflect the bunches of premisses on which a conclusion

Australasian Journal of Logic (18:5) 2021, Article no. 4 
might depend, at a given stage of a "natural deduction," our compounding principles are at the same time bunching principles. $\wedge I$, since it introduces a conclusion extensionally bunched by $\wedge$, goes with an ordinary conjunction of tags by $\wedge$. On the other hand, we might well expect that a rule $\circ I$, which fuses conclusions (and which hence makes them available for joint use in a relevant sense) would go with a fusion of tags. So indeed it does.

So much for the psychological viewpoint. What is the formal one, based on our straightforward understanding of assertions $A \leq B$ as asserting provable implications of R? The question, accordingly, is one of what we can conclude, with respect to a specific connective, when it is given (by theorems of $\mathrm{R}, \mathrm{RQ}$, or $\mathrm{R}^{\sharp}$ ) that both $A \leq B$ and $A^{\prime} \leq B^{\prime}$. The principal part of the answer is given, for our purposes here, in virtue of the following theorem schemes that reflect compounding principles.

$$
\begin{array}{ll}
C M \wedge . & (A \rightarrow B) \wedge\left(A^{\prime} \rightarrow B^{\prime}\right) \rightarrow . A \wedge A^{\prime} \rightarrow B \wedge B^{\prime} \\
C M \vee . & (A \rightarrow B) \wedge\left(A^{\prime} \rightarrow B^{\prime}\right) \rightarrow . A \vee A^{\prime} \rightarrow B \vee B^{\prime} \\
C M \circ . & (A \rightarrow B) \circ\left(A^{\prime} \rightarrow B^{\prime}\right) \rightarrow . A \circ A^{\prime} \rightarrow B \circ B^{\prime}
\end{array}
$$

In view of $\wedge \circ$ above, $C M \circ$ continues to hold if $\circ$ is traded in for $\wedge$ as the principal connctive in its antecedent. (However, the stronger form is worthy of note, while it is also worth noting that $\wedge$ cannot be so traded in for $\circ$ in the antecedents of $C M \wedge, C M \vee$.)

Our compounding principles lead immediately to the following "natural deduction" rules:

$$
\begin{array}{ll}
\wedge I . & \Gamma \leq A \& \Delta \leq B \Rightarrow \Gamma \wedge \Delta \leq A \wedge B \\
\vee E . & \Gamma \leq A \& \Delta \leq B \Rightarrow \Gamma \vee \Delta \leq A \vee B \\
\circ I . & \Gamma \leq A \& \Delta \leq B \Rightarrow \Gamma \circ \Delta \leq A \circ B
\end{array}
$$

These are the basic multi-premiss compounding principles. Note, incidentally, that the middle principle gets called $\vee E$, and gets taken conceptually as an elimination rather than as an introduction rule. But this is just to make contact with familiar systems of natural deduction; formally, $\wedge I$ and $\vee E$ are, in their fundamental forms, analogous, a fact which tends to get hidden in some conceptual frameworks.

Normally, a multi-premiss rule will involve both some compounding operation and some further inference. A crucial example is 
$\rightarrow E . \quad \Gamma \leq A \rightarrow B \& \Delta \leq A \Rightarrow \Gamma \circ \Delta \leq B$

(The reader who reflects on the $[\mathrm{ENT}]$ form of $\rightarrow E$ will now make some further sense of our claim that o does the systematic job that $\cup$ does, in an auxiliary sense, in $[\mathrm{ENT}]$.)

We can break the $\rightarrow E$ rule into 2 parts. First, given its premisses, we can conclude immediately by $\circ I$,

$$
\text { (xxvi) } \quad \Gamma \circ \Delta \leq(A \rightarrow B) \circ A
$$

Moreover, by reflexivity of $\leq$ and $\rightarrow I$, we have immediately

$$
\rightarrow \circ E . \quad(A \rightarrow B) \circ A \leq B
$$

Whence from (xxvi) and $\rightarrow \circ E$ we conclude by directed replacement

$$
\text { (xxvii) } \Gamma \circ \Delta \leq B
$$

which is the conclusion of the $\rightarrow E$ rule above.

This suggests that, from a formal viewpoint, we can, in applying a multipremiss rule, break that application into a compounding stage (e.g., that reflected in (xxvi)) and a replacement stage (e.g., which led just above from (xxvi) to (xxvii), given $\rightarrow \circ E$ ). Note that the replacement stage is, from a psychological natural deduction point of view, encompassed in a 1-premiss rule (e.g., we view (xxvi) as a "tagged formula" and $\rightarrow \circ E$ as a "rule," as in the 1-premiss cases above).

This casts light, too, on what is systematically involved in the motivating "use criterion" of [ENT]. For [ENT] counts such use as "use in an application of modus ponens for $\rightarrow$." In some sense, this is backward; the key use involved, systematically speaking, is one of $\circ I$, which signals that we are going to concern ourselves with formulas tightly bunched. And then it is a matter of some choice what we are going to claim obtains in this case; we lay down what follows from a tight bunch of premisses (as, for that matter, we lay down what follows from premisses loosely bunched by $\wedge$ ). Whether what we have laid down is correct, or plausible, is not a matter which the system itself can adjudicate. We adjudicate it, by deciding to employ the system (or by refusing to employ it), given our informal goals, insights, and sense of "fit." And we do not adjudicate it by referring on indefinitely to further 
formal systems, formal semantics, and formal whatnot. For each of these devices raises the same questions of correlation and reflection of informal purpose that the original system raised.

Again, in all of this, use is not univocal. There are uses and uses. In particular, from the $\mathrm{R}$ viewpoint, there is tight use, in which all the premisses must collaborate, so to speak, to yield a conclusion tightly derived from them, and loose use, in which that degree of collaboration is not required. This clears up something in the debate as to whether we should "switch" to relevant logics. What is involved, in fact, is not so much of a "switch" as a provision to make formal room for various argumentative situations that arise informally. The C-defender tells us that one kind of provision does for everything; that is a priori unlikely, and a posteriori false, except on attenuated epicycular distortion of those features of $C$ that attract us to it in the first place. But the R-defender is ill advised to set out to start a New Church. It will suffice to revoke some of the indulgences issued by (and requested for) the old one, and to restore formal insight, in its various manifestations, to its proper role as the servant and the guide of informal purpose, not its master.

In particular, though we have here 2 kinds of bunching connectives, $\wedge$ and $\circ$, it is not ruled out that there should be more, or that the ones which we have might be assigned different properties. Nor is it the case, despite all the priority which I have given to informal purpose, that such decisions should be referred too simply to informal intuition. The collaboration between purpose and system is 2-way, not 1-way, and the formalizing process would lose most of its charm if all that was sought was a pedantic record of what, informally, we say and think anyway. For that matter, it would lose most of its utility as well. Response to existing human needs and a spirit of free creation intermingle in all intellectual enterprises worth pursuing. It is well that they should do so in logic also.

We spoke above of $\rightarrow E$ as though it were almost optional for R. In fact, the concern for a proper deduction theorem reflected above in the principle $\rightarrow I$ makes it by no means optional; indeed, as we saw in breaking it into stages, $\rightarrow E$ follows when we are given $\rightarrow I$ and $\circ I$. But our general principle remains; $\mathrm{R}$ is motivated whole, and we cannot account individually for the motivation of its parts without taking account of relations of systematic interdependence.

So compounding principles have joined our general concern for replacement and deduction theorems, in the analysis of what makes natural deduc- 
tion tick. Let us now turn to some other multi-premiss rules that may prove useful in actual derivations. One of the big headaches of natural deduction turns often on $\vee E$ rules that may prove useful in actual derivations. One of the big headaches of natural deduction turns often on $\vee E$ rules, which are systematic counterparts of arguments by cases. We gave a basic form of this rule above. But, typically, we want to apply $\vee E$ when we have already derived $C \vee D$, on some assumptions reflected in a tag $\Theta$. So let us take, in complicating $\vee E$ to account for this situation, some further matters to heart.

The central further matters involve distribution. Recall that each of $\circ$, $\wedge$, together of course with $\vee$ itself, distributes over $\vee$ on R-related insights. (Caution: $\rightarrow$ does not so distribute.) This allows us to combine the $\vee E$ rule above with a bit of distributive tag manipulation. Pursuing such thoughts leads to the following useful forms of the $\vee E$ rule.

$$
\begin{array}{ll}
\vee E^{\prime} . & \Gamma \leq C \vee D \& \Gamma \wedge C \leq A \& \Gamma \wedge D \leq B \quad \Rightarrow \quad \Gamma \leq A \vee B \\
\vee E^{\prime \prime} . & \Gamma \leq C \vee D \& \Delta \circ C \leq A \& \Delta \circ D \leq B \quad \Rightarrow \quad \Gamma \circ \Delta \leq A \vee B
\end{array}
$$

$\vee E^{\prime \prime}$ corresponds to the [ENT] form of the $\vee E$ rule. It may be generalized as follows. Let $f[p]$ be any tag built up from the atom $p$ and other fixed formulas $A_{1}, \ldots, A_{n}$ on (possibly iterated) compounding by $\circ, \wedge, \vee$. Let $f[C]$, $f[D], f[\Gamma]$ result respectively from the replacement of a specific occurrence of $p$ in $f[p]$ with $C, D, \Gamma$; then we have in general the rule

$$
\vee E^{\star} . \quad \Gamma \leq C \vee D \& f[C] \leq A \& f[D] \leq B \quad \Rightarrow \quad f[\Gamma] \leq A \vee B
$$

This generalization is suggested by Dunn's Gentzen system.

Two points may be noted about the above rules. First, we have felt free to mix $\Gamma, \Delta$ on the one hand and $C, D$ on the other in what we have been suggesting to the reader is "tag" position. There is, formally, no harm in that. And, psychologically, there is some gain. Consider the $\vee E^{\prime}$ rule. The idea is that, given that we have derived a "tagged formula" $C \vee D$, under certain assumptions signalled by the "tag" $\Gamma$, we may eliminate $C \vee D$ by cases on adding first $C$ and then $D$ to our stock of fixed assumptions. $\vee E^{\prime \prime}, \vee E^{\star}$ rest on similar points, though they give us a bit more manipulative freedom, at some cost in perspicuity. (But it is this cost in perspicuity which makes the $[\mathrm{ENT}] \vee E$ rule unmemorable, and which will lead us in practice to prefer the simpler rules $\vee E, \vee E^{\prime}$. But any of these rules, in use, will be called simply $\checkmark E$. None of them, of course, need be remembered, except the original tag-

Australasian Journal of Logic (18:5) 2021, Article no. 4 
compounding and simple $\vee E$, for each of $\vee E^{\prime}, \vee E^{\prime \prime}, \vee E^{\star}$ is easily worked out on our suggested plan of splitting multi-premiss rules into compounding steps and replacement steps. On the other hand, since arguments by cases are quite important in practice, a few finger exercises to determine what goes in $\mathrm{R}$ and what doesn't will do the reader no harm. And, if he keeps in mind the simple distribution laws mentioned above, he will, without undue strain on his memory, quickly get the formal intuitions down. While, since $\vee$ is not primitive for our formulation of $\mathrm{R}^{\sharp}$, no intuitions regarding it are required, they are to the point when we are thinking seriously about, say, numbers.)

Second, the reader will have noted that none of the above rules actually eliminate $\vee$. But that's a small point; if, in the rules above, $A, B$ are the same formula, $\vee$ may be eliminated by idempotence. And, in arguing by cases, one often gets down to more cases, which is reason enough to leave the above rules as they stand. And further modification (e.g., so that we don't always have to use the same $\Gamma$ in $\vee E^{\prime}$, as we argued against always being stuck with the same $\Gamma$ in applying $\wedge I$ above) will readily occur to the reader.

Having thought that much about $\vee E$, let us use what we have thought to get a useful and correct form of the principle $\exists E$ of existential quantifier elimination (i.e., existential instantiation). Our original $\vee E$ was a compounding principle. For quantifiers, such compounding principles require only 1 premiss and are rather trivial. For we get without toil the rules

$$
\begin{array}{ll}
C M \forall . & \Gamma \leq A \Rightarrow \forall X \Gamma \leq \forall X A \\
C M \exists . & \Gamma \leq A \Rightarrow \exists X \Gamma \leq \exists X A
\end{array}
$$

on easy application of principles given above. And, since tags are formulas, this introduction of quantified tags makes sense.

Now, concentration on $C M \exists$, let us copy the move which took us from $\vee E$ to $\vee E^{\prime}$ above. It produces

$$
\exists E^{\prime} . \quad \Gamma \leq \exists X A \& \Gamma \wedge A \leq B \quad \Rightarrow \quad \Gamma \leq \exists X B
$$

To apply $\exists E^{\prime}$, it is evidently required that $X$ must not occur free in $\Gamma$. And, for $\exists E^{\prime}$ actually to eliminate an existential quantifier, it suffices that $X$ not be free in $B$ either, whence the vacuous quantifier on $B$ may be dropped (compare our discussion of the conditions under which $\vee E$ actually eliminates a $\vee$ ).

Skipping an intermediate stage corresponding to $\vee E^{\prime \prime}$, let us move im-

Australasian Journal of Logic (18:5) 2021, Article no. 4 
mediately to the appropriate generalization of $\vee E^{\star}$ in this case. We require that $X$ should not occur free in $f[p]$. Then

$\exists E^{\star} . \quad \Gamma \leq \exists X A \& f[A] \leq B \quad \Rightarrow \quad f[\Gamma] \leq \exists X B$

The same restrictions as before on building up $f[p]$ are in force. And we shall henceforth refer to any of $C M \exists, \exists E^{\prime}, \exists E^{\star}$ simply as $\exists E$, making minor moves (e.g., dropping a vacuous quantifier) without specific comment, and concentrating on the simple rules $C M \exists, \exists E^{\prime}$.

We have now dealt with all major principles that are standardly involved in systems of natural deduction except for indirect proof. But this also takes a particularly simple form in $\mathbf{R}$. Having added $\mathbf{t}$ conservatively, and hence f, recall our "quasi-definition" above on which $\sim A$ is linked equivalently to $A \rightarrow \mathbf{f}$. Combined with $\rightarrow I$, this yields

०5. $A \circ B \rightarrow \mathbf{f} \simeq A \rightarrow \sim B$

And this motivates the following rule, on our old 1-premiss principles.

$$
\sim I . \quad \Gamma \circ B \leq \mathbf{f} \Rightarrow \Gamma \leq \sim B
$$

Given double negation, we may indifferently apply this rule in the alternative form

$$
\sim I^{\prime} \quad \Gamma \circ \sim B \leq \mathbf{f} \Rightarrow \Gamma \leq B
$$

As in the case of $\rightarrow I$, we may dress up an application of $\sim I$, in either form, to give the psychological impression that we have assumed a "hypothesis" for "reductio ad absurdum." In that guise, this 1-premiss rule also looks like a 2-premiss rule: e.g.,

$$
\sim I . \quad \Gamma \circ \alpha \leq \mathbf{f} \& \alpha=B \Rightarrow \Gamma \leq B
$$

As before, the second premiss of this rule is just window dressing formally, though psychologically useful.

These are the basic forms of indirect proof in $R$, making use of the technical convenience of $\mathbf{f}$. But we can, of course, do without $\mathbf{f}$. Doing without f may, however, most simply be referred to the following three principles of f-introduction.

Australasian Journal of Logic (18:5) 2021, Article no. 4 


$$
\begin{array}{ll}
\mathbf{f} I . & A \circ \sim A \leq \mathbf{f} \\
\mathbf{f} I^{\prime} . & A \wedge \sim A \leq \mathbf{f} \\
\mathbf{f} I^{\prime \prime} . & (A \rightarrow \sim A) \circ A \leq \mathbf{f}
\end{array}
$$

Each of these principles is trivially established; we take the first as basic, but refer (on our usual practice) to them collectively as $\mathbf{f} I$. And note that they reflect respectively our reductio principles $R D C, R D B, R D A$ above. And what the basic principle $\mathbf{f} I$ suggests is the following form of $\sim I$.

$$
\sim I^{\star} . \quad \Gamma \circ B \leq A \& \Delta \leq \sim A \Rightarrow \Gamma \circ \Delta \leq \sim B
$$

Given double negation, we have various forms of $\sim I^{\star}$ also. Moreover, it too can be dressed up for psychological purposes by adding an extra premiss as "tagged hypothesis," just like $\sim I$ above.

Note that, so far as use is concerned, there is a curious feature to $\sim I^{\star}$. We only have to use $B$ to get one-half of the contradiction between $A, \sim A$. (But this is, as Dunn points out, just modus tollens under hypotheses; compare the $\rightarrow E$ rule above.)

All of our forms of $\sim I$ will be referred to simply as $\sim I$, while we leave to the reader the formulation of reductio principles corresponding to the weaker forms of $\mathbf{f}$-introduction. This is somewhat interesting, independently, in view of our parenthetical remark just above. For, while R-insights are very much tied to modus tollens (reflected, systematically, in an attachment to contraposition), weaker relevant logics do without this principle (in a way adaptable to natural deduction technique). So, for that matter, does the classical semantical negation investigated by Urquhart and by Routley and me.

This brings up a more general point. Our general scheme of natural deduction is easily adaptable to other relevant logics. But, in analyzing indirect proof, it is rather more convenient to concentrate rather on specific properties of $\mathrm{R}$. The most important of these are (i) that $\sim A$ is definable as $A \rightarrow \mathbf{f}$ and (ii) that $A \circ B$ is definable as $\sim(A \rightarrow \sim B)$. Neither (i) nor (ii) is true even in $\mathrm{E}$, to say nothing of the weaker systems.

Some objections have been lodged to these points (i) and (ii). The essence of the objection to (i) is that if $\sim A$ is defined in $\mathrm{R}$, the double negation principle $\sim \sim A \rightarrow A$, which now becomes a principle of the theory of deduction, is no longer plausible; neither is the law $A \vee \sim A$ that follows from it. (This line is taken, e.g., by the paper contributed by Urquhart to [PRL].) The Australasian Journal of Logic (18:5) 2021, Article no. 4 
essence of the objection to (ii) is that $\circ$, which is a kind of conjunction, is ill-served by being taken, formally, as a kind of consistency. (E.g., Harper has raised in conversation the following kind of objection. It is the case that the axiom of choice, together with other axioms of set theory, implies, say, Zorn's lemma. Let us symbolize these respectively as $A C, O A, Z L$. On the use criterion, we might well expect

$$
\text { (1) } A C \circ O A \rightarrow Z L
$$

to be a theorem of a relevant set theory. Moreover, it is known that the axiom of choice is consistent with the other axioms; i.e.,

$$
A C \circ O A
$$

Putting (1) and (2) together with $\rightarrow E$, this seems to suggest that we can derive

(3) $Z L$

outright, perhaps without the benefit of any axioms at all.)

To both of these objections, one must answer that the deductively classical character of the negation $\sim$ of $\mathrm{R}$ has not been sufficiently appreciated or understood. Consider, to begin with, the putative "deduction" of (3). And let us transfer it to a wholly classical context. In that case $\circ$ just collapses to ordinary $\wedge$, both on deduction-theorem considerations and on the definition of $A \circ B$ as $\sim(A \rightarrow \sim B)$, where $\rightarrow$ is in this last case material. So put, classical conjunction is also a kind of consistency. But it is a material consistency, which is of a very strong kind. I.e., $A$ is in this material sense consistent with $B$ just when both are in fact asserted. And, on such classical thoughts, there is no objection at all to (1) above. But (2), standing alone, won't do. Gödel, certainly, did not prove both the axiom of choice, and the other axioms of set theory, from scratch; that, indeed would have taken some doing. But what he did do was to construct a model of $O A$ in which (2) is true. In the model, $Z L$ is also of course true. But there is nothing shocking about that. To be sure, this establishes (insofar as it can be trusted) that $O A \rightarrow \sim A C$ is not a theorem of logic; but this is a far cry from establishing that $\sim(O A \rightarrow \sim A C)$, i.e., $O A \circ A C$ is a theorem of logic, or even that it is a theorem of the proper theory determined classically by $O A$. Of course it is

Australasian Journal of Logic (18:5) 2021, Article no. 4 
not, as Cohen showed (insofar as one can trust him).

Now let us transfer these thoughts to an R-context. $A \circ B$ means, when asserted in an R-theory, that $A$ and $B$ are relevantly consistent in the same sense that $A \wedge B$ means that $A$ and $B$, classically, are materially consistent; i.e., that $\sim(A \rightarrow \sim B)$ is a theorem. Again, this must be sharply distinguished from the mere unprovability of $A \rightarrow \sim B$. This is a much weaker sense of consistency. And, on analogies to other relevantly formulated theories, one would suppose that, if a relevant ZF were formulated, its theorems would all be classical theorems (though not conversely) on a direct trade-in of relevant $\rightarrow$ everywhere for material $\supset$. And, in this case, exactly the classical situation would obtain; i.e., $O A \rightarrow \sim A C$ is a presumptive non-theorem of relevant logic, and even of the relevant set theory determined by $O A$. But its negation, namely (2), is also a presumptive non-theorem, both logically and in the theory determined by $O A$. Of course, trivially, (2) is, both classically and relevantly, a theorem of the theory determined by both $O A$ and $A C$. And, while relevant model theory has not proceeded to ZF, presumptively one can use the Gödel technique to cook up, relevantly, the Gödel model of $O A$ that makes $A C$ true.

However, the relevant theory does open one interesting possibility that the classical theory cannot accommodate, and which might make interesting sense of Harper's "counterexample." For, set theory being a highly extensional subject, it is not all that clear that (1) is wanted relevantly. Perhaps we should just want it with $\wedge$ standing in the antecedent instead of $\circ$. In that case, we could transfer the Gödel result to an object theory by taking (2) as an additional axiom of a theory in which $O A$ and $\sim A C$ appropriately formulated, are already theorems. But this is speculative, and I shall not pursue it; my guess is that relevant insights will give rise to their own set theories, which may lead both to alternative ways or formulating traditional set theories and perhaps to simplified ways of reaching old results. The point to be stressed here is that, however one looks at it, the claims of (2) to be an object theorem must be independently justified, and cannot rest merely on the unprovability of its negation.

And now let us move this discussion of the objection (ii) above to a more general level. There is a precious sense in which we wish to say that $A$ is consistent with $B$, really, just when $A$ does not entail $\sim B$. And this ties in with a pet idea that Anderson used to express, and which finds its way partially into [OPP]: namely, that the true entailments are exactly what they are, on formal grounds, and that when an entailment is not true, its negation 
is true. From the viewpoint of concrete theories formalizing some subject matter, this suggests that such theories should be complete on the class of formulas $A \rightarrow B$, where $\rightarrow$ expresses entailment; i.e., for each $A$ and $B$, exactly one of $A \rightarrow B, \sim(A \rightarrow B)$ should be a theorem.

I do not know to what degree this Andersonian ideal can be approached, in formulating theories based on some non-logical subject matter, or even in formulating logic itself. After all, there is a certain preference for theories that are consistent and complete on all formulas without free variables, but we find them hard to come by. But I do know that it is not an ideal which is applicable to R. For R, as I learned from Belnap, and have repeated often, does not express entailment. It indicates entailment, in the sense that, when $A \rightarrow B$ is a theorem of $\mathrm{R}$, the $\mathrm{R}$-view is that $A$ entails $B$. Exactly the same point can be made in the classical case; the $\mathrm{C}$-view of when $A$ entails $B$ may be a little strange, but, as Quine has often noted, it is quite clear; and it has nothing to do with taking material implication as a kind of entailment, or even a strict implication.

In particular, when we are formalizing some non-logical subject matter, material implication ceases to indicate classical entailment. If, e.g., we have proved $A \rightarrow B$ in $C^{\sharp}$, that doesn't mean that $B$ is a logical consequence of $A$; it's just an arithmetical consequence, insofar as $C^{\sharp}$ formalizes classical arithmetic. (Such matters, alas, tend to get mixed up in claims that mathematical and logical truths share the property of being analytic; while, however, I have to hold that the dragons have got St. Willard rather more often than he has got them, the notch on his tunic for having done in the Beast of Analyticity in [TDE] is deserved, and the confusions caused by that Beast will not be recommended, or even further noticed, here, save to note that the slaying of the Beast may be bad for the Andersonian ideal. But I am not pursuing that ideal here anyway; and if I were, in contexts other than R, I should be doing so in a system-dependent way, relativizing the Andersonian ideal, respecting [TDE], and giving aid and comfort to some of the fine, upstanding, law-abiding dragons whom the author of [TDE] too easily maligns.)

Just so, when $A \rightarrow B$ is a theorem of $\mathrm{R}^{\sharp}$, or other theory based on $\mathrm{R}$, this is to be taken not as a logical but as a non-logical (indeed, an arithmetical) kind of implication. In exact analogy, the theoremhood of $A \circ B$, though it states (in R) some kind of consistency, is not in $\mathrm{R}^{\sharp}$ a statement of logical consistency. It is a statement, rather, of arithmetic compatibility. And that's a useful notion, which stands reasonably between the C-insistence that such compatibility is just ordinary $\wedge$, and the (presumptive) E-insistence that 
arithmetic compatibility is at root logical compatibility.

From the beginning, all parties have held that relevant implication, in the sense of $\mathrm{R}$, is not entailment. Some of us have expected that relevant implication might well turn out to be more interesting and useful than entailment. (But, as Belnap has said in another context, the jury is apt to be out for a while on that one.) For our grounds for holding, in real life, that $A$ implies $B$ are unlikely to be explicated either in terms that are wholly formal or in those that are wholly material. If so, neither waving systems of logic nor truth-tables at real life implication is going to capture it. Rather, we are going to be thrown back on pre-theoretic convictions (to be reflected in the non-logical axioms of a concrete theory) to ground the implication statements that we accept, and to separate them from those which we reject. For while we may expect $\mathrm{R}$ (or some other relevant logic) to furnish the logic of implication, knowing the logic does not tell us which implication statements are true, save for those few that are logically true.

And, on this point, much discussion of implication has just veered north or south of the central matter. (And much of this discussion, I fear, has shown undue respect for the Beast which St. Willard slew.) For people have indulged themselves in so parsing the word 'Implies' (and the rough synonyms thereof that enter into such discussions) so that it turns out either wholly formal (e.g., validity of the material conditional) or wholly material (e.g., the material conditional itself), with calumny thereupon heaped upon the other side for failing to understand simple logical points. But the whole discussion has been vitiated by the unwillingness of almost everybody to take into account simple pre-logical points - namely that, taken together, both formal and material considerations underdetermine the stock of honest implications that we use to tie together actual theories. And there is just no point in dwelling on the finely spun stories about how, deep down, formal or material considerations rule anyway, at least in principle. For that is just to prolong the debate at its most useless point: namely, the point at which project is mistaken for accomplishment, while reductions which no one has seen are taken for granted.

As goes relevant implication, so goes relevant consistency. In $\mathrm{E}$, where consistency is taken in a logical sense, it is not identified with fusion. In C, where consistency is taken in an outright material sense, it is identified with fusion, which is just $\wedge$ there. $R$ veers to the $C$-side on this point, save that $\circ$ is no longer $\wedge$. On its intuitions, that is exactly where it belongs.

Similar remarks are to be made about point (i) above; namely, the de-

Australasian Journal of Logic (18:5) 2021, Article no. 4 
finability of $\sim A$ in $\mathrm{R}$ as $A \rightarrow \mathbf{f}$. It is the definability which establishes the negation of $\mathrm{R}$ as a deductive kind of negation, as it is the holding of classical negation laws which establishes $\sim$ as a classical kind of negation. So $\sim$ is deductively classical.

Now being deductively classical is not, for my money, the same thing as being semantically classical. (Belnap, however, suggests in correspondence that there is a point of view on which $\sim$ in $\mathrm{R}$ turns out to be semantically classical also; I have my doubts, but am not sufficiently acquainted with his latest thoughts to close off that point of view completely.) In fact, to be semantically classical, for my part, I take as subscribing to an article of faith; namely, that our semantic intuitions about negation are clear. When convenient, I will buy that article of faith, though in view of the set-theoretic and semantic paradoxes I never feel truly at ease in that faith. To be deductively classical I take in a weaker sense: namely, that we will proceed as though our article of semantic faith were justified. Thus, e.g., we will buy laws of double negation, non-contradiction, excluded middle, and so forth. But we will not buy them with a blank cheque. I.e., consider the law of excluded middle. This expresses, in one perspective, a kind of Peircean conviction that the world is complete in the long run, or at least that our theories about the world are to be taken as thus potentially complete. By adopting a law of excluded middle, we tie ourselves to that conviction, asserting $p \vee \sim p$ even without grounds to assert $p$ or $\sim p$ independently. Similarly, in asserting double negation, we take it as sufficient verification of $p$ when the claim that $p$ relevantly implies trouble itself runs into trouble.

However, the deductive viewpoint leaves open one possibility that the semantic viewpoint forecloses; namely that, in certain circumstances, we shall run into trouble. Systematically, that means, formalizing the circumstances in question, that we get a proof of $\mathbf{f}$. We hoped that that wouldn't happen; it runs contrary to our semantic faith. We hope moreover that, when it does happen, there is something unusual about the circumstances, that we can fix up. But it's good also to carry on resolutely in tough times, making what we can of our temporarily undone faith without accepting thereby the total shattering of the insights that we sought to formalize.

Maybe the semantic faith, ultimately, just won't do. In that case, $\mathrm{R}$ should cancel $\sim \sim A \rightarrow A$, or at least restrict to those situations in which it will do. Maybe the semantic faith will always do; in that case, $\mathrm{R}$ is "chicken" for lacking $A \wedge \sim A \rightarrow B$. But the intermediate possibility is that we do not know how far, and in what circumstances, our semantic faith will carry us. 
As a regulative principle, we accept it. As a blank cheque, we do not. So $\mathrm{R}$ understands $\sim A$ via the assertion that $A$ implies something objectionable; itself, for example, if $A$ is objectionable. And $\mathrm{R}$ understands moreover that there will be non-trivial theories with objectionable theorems; the weak case for this understanding is that we may entertain such theories for pragmatic reasons; the strong case is that, in matters of metaphysical or dialectical depth, we shall have no option but to entertain them. (I have put, mainly, the weak case here, but I have some attachment to the strong one also; but I am not insisting on the latter attachment here.) Even in the midst of this understanding, however, $\mathrm{R}$ hews to our ordinary processes of reasoning where negation is concerned. This may have the effect of isolating the inconsistent portions of a theory, and thus give us hints for its consistent repair; or perhaps in practice, of simply ignoring those portions of a theory, to pursue other matters; e.g., despite the overall inconsistency of Newtonian theory and physical observation, it is good enough for moon shots, even if it isn't good enough for shooting at the perihelion of Mercury a century hence. The computers at NASA don't care about that now, and a theoretical perspective which discards irrelevant difficulties has something, accordingly, to be said for it.

So we shall stick to our R-perspective regarding negation, and its resultant simplification of a general relevant theory of indirect proof. And now we turn to one final matter respecting our general theory of deduction. We have taken deduction deductively. In particular, this means that when we have bunched premisses for the purpose of deduction, we have bunched them finitely (pairwise, in fact, though the finitary generalizations are obvious).

A popular view, however, is that logical consequence itself is a semantic notion. On that view, it is natural to take such consequence as a relation between a set $S$ and a formula $A$ (or perhaps another set $T$ ), such that this relation holds unless it is the case that all members of $S$ are true and $A$ (all members of $T$, alternatively) is false. (One takes Tarski to have popularized this view, together with the related view that logical consequence is an operation (though not necessarily of a semantic kind) on sets.)

Our view seems to be somewhat different. In the first place, we look not only to the premisses collected, but to the manner of their collection, in order to decide what follows from them. Second, we only provide for finite collecting processes. And third, the notion of consequence which results is at all points system-dependent.

In fact, our view is merely more general. In the finitary case, the classical

Australasian Journal of Logic (18:5) 2021, Article no. 4 
view merely amounts, as we have seen, to bunching the members of the above $S$ by $\wedge$, and of $T$ by $\vee$ on its parenthetical extension, and letting the relation $\leq$ be $\mathrm{C}$-entailment. The infinitary case, in a securely classical firstorder perspective, is reduced to the finitary one by the classical compactness theorem; it is, so to speak, an added extra. In the more intricate situations in which compactness fails, so that properly infinitary collections of premisses are considered, we have two things to say. First, insofar as we are concerned with actual argument, it is perforce finitary; extensions to infinitary cases are imaginative extensions. Second, there is no objection in principle to a similar imaginative extension of the present account. If we can collect, in the process of set formation, infinitely, we can conjoin infinitely. There is no reason why we cannot fuse infinitely. We are not interested, here, in doing so; indeed, to do so would seem to be a bit of an arcane diversion. But such diversions may be pursued as one will.

Finally, let us concentrate on the third of the above points; namely, that our account is system-dependent. So, I hold, is the classical one; indeed, it is dependent on a certain mythology with regard to truth and falsehood that seems to me productive of great evils. But let that pass. The question is whether anything but truth and falsehood enters into the notion of consequence. The $\mathrm{C}$-view is that it does not. And we can accommodate that view: directly, at the lower levels of logic. But we are also prepared for an affirmative answer; for one thing, an affirmative answer is the pre-theoretic one, and we are not convinced by claims made for its post-theoretic negation. And, once we are off the terrain in which the notion of consequence is Godgiven, we are not in search of another god to give us another notion. Let us see what notions there are. And of course that inquiry is system-dependent; while mathematical theology, perhaps, will find new reasons for divine preferences among systems, we leave that enterprise, for now, in the hands of those who have been confounded by it so far.

Let us turn now from such philosophical reflections to a concrete demonstration of how our natural deduction system works in practice. E.g., let us derive, for practice, a set of axioms sufficient for the pure implicational fragment of R. Since it would suffice, given the non-independent character of this system of natural deduction, just to write those axioms down, let us make the problem a little hard by allowing ourselves only principles of tag introduction (i.e., hypothesis introduction, psychologically), tag replacement (on the $\mathrm{R}$ properties of fusion), and our rules $\rightarrow E, \rightarrow I$.

Australasian Journal of Logic (18:5) 2021, Article no. 4 


\begin{tabular}{|c|c|c|c|}
\hline 1. & $\alpha=$ & $A$ & HYPOTHESIS \\
\hline 2. & $\mathbf{t} \circ \alpha \leq$ & $A$ & 1, to, Tag Replacement \\
\hline 3. & $\mathbf{t} \leq$ & $A \rightarrow A$ & $1,2, \rightarrow I$ \\
\hline 4. & $\beta=$ & $A \rightarrow B$ & НYPOTHESIS \\
\hline 5. & $\beta \circ \alpha \leq$ & $B$ & $4,1, \rightarrow E$ \\
\hline 6. & $\alpha \circ \beta \leq$ & $B$ & $5, C \circ$, Tag Replacement \\
\hline 7. & $\alpha \leq$ & $A \rightarrow B \rightarrow B$ & $4,6, \rightarrow I$ \\
\hline 8. & $\mathbf{t} \leq$ & $A \rightarrow . A \rightarrow B \rightarrow B$ & $\begin{array}{l}\text { 7, to, Tag Replacement, } \\
1, \rightarrow I\end{array}$ \\
\hline 9. & $\gamma=$ & $B \rightarrow C$ & HYPOTHESIS \\
\hline 10. & $\beta \circ \gamma \circ \alpha \leq$ & $C$ & $\begin{array}{l}9,5, \rightarrow E, A \circ, C \circ \text {, Tag Re- } \\
\text { PlaCEMENT }\end{array}$ \\
\hline 11. & $\beta \circ \gamma \leq$ & $A \rightarrow C$ & $1,10, \rightarrow I$ \\
\hline 12. & $\beta \leq$ & $B \rightarrow C \rightarrow . A \rightarrow C$ & $9,11, \rightarrow I$ \\
\hline 13. & $\mathbf{t} \leq$ & $A \rightarrow B \rightarrow . B \rightarrow C \rightarrow . A \rightarrow C$ & $\begin{array}{l}\text { 12, to, TAG REPLACEMENT, } \\
4, \rightarrow I\end{array}$ \\
\hline 14. & $\delta=$ & $A \rightarrow A \rightarrow B$ & НYPOTHESIS \\
\hline 15. & $\delta \circ \alpha \leq$ & $A \rightarrow B$ & $14,1, \rightarrow E$ \\
\hline 16. & $\delta \circ \alpha \circ \alpha \leq$ & $B$ & $15,1, \rightarrow E$ \\
\hline 17. & $\delta \circ \alpha \leq$ & $B$ & $\begin{array}{l}16, \quad S Q \circ, \quad A \circ, \quad \text { TAG RE- } \\
\text { PLACEMENT }\end{array}$ \\
\hline 18. & $\delta \leq$ & $A \rightarrow B$ & $17,1, \rightarrow I$ \\
\hline 19. & $\mathbf{t} \leq$ & $(A \rightarrow . A \rightarrow B) \rightarrow . A \rightarrow B$ & $\begin{array}{l}\text { 18, to, TAG Replacement, } \\
14, \rightarrow I\end{array}$ \\
\hline
\end{tabular}

We have presented this as a deduction of "tagged formulas" (which accounts for our curious habit of putting the main relations $\leq$, = right up against the Greek letters; in fact, the symbols for the main relations could be left out altogether, since $=$ always goes with a НyPOTHEsis step and $\leq$ with anything else). We have also been unusually explicit in calling attention to TAG REPLACEMENT principles, including those that introduce $\mathbf{t}$ on a formula that would otherwise be untagged after an application of $\rightarrow I$.

It is well-known that the tagged formulas $3,8,13,19$ above suffice for the derivation of all pure implicational theorems of $\mathrm{R}$, with $\rightarrow E$ as rule. And the reader is invited to verify, accordingly, that all such theorems will be derivable in our "natural deduction system" on the limitations imposed for

Australasian Journal of Logic (18:5) 2021, Article no. 4 
the sake of practice. He is also invited to pick and choose among the other rules so as to derive all theorems of $R$, and of $R Q$, tagged just with $\mathbf{t}$, from a limited basis of "natural deduction" rules.

For readers familiar with the $[\mathrm{ENT}]$ account (other readers being free to skip this part), let us compare the proofs of pure implicational theorems offered above in "natural deduction" style with those of [PCE] and [ENT]. There are some inessential differences. E.g., we "tag" formulas derived with Greek letters, much as [ENT] uses sets of Arabic numerals. And we tag theorems of $\mathbf{R}$ with $\mathbf{t}$ as [ENT] tags them with the empty set.

Most strikingly, we have, as a matter of form, ditched Fitch as promised; i.e., the nested subproofs which play such a striking part in [ENT] are absent here. (Fitch, I hope, won't mind.) This has the important advantage of linearizing our "natural deductions," making redundant complex rules of "reiteration" of formulas from one subproof to another.

Another feature of the present treatment is that formulas assumed here on "hypothesis" are never finally discharged; we may go on using them as many times as we wish, even after applications of $\rightarrow I$. (Was it not, perhaps, a little surprising that, after "discharging" the hypothesis of step 1 in step 3 , we nevertheless managed to "use" this hypothesis to justify 6 further steps in the above derivation?)

In fact, as the reader will recall from our discussion above of $\rightarrow I$, it is, deeply speaking, a 1-premiss rule, not a 2-premiss rule. (That much, indeed, is just Gentzenish thinking.) So a hypothesis may hang around indefinitely, and be used as often as one pleases, without doing any damage. (Indeed, on the conventions here, damage could occur only if we abbreviated 2 distinct hypotheses with the same Greek letter; that, evidently, is a no-no.)

Having hypotheses linger on has a strangely irrelevant flavor. In fact, it is the tags which suffice for relevance. If a hypothesis bears tag $\alpha$, the only steps which depend upon this hypothesis are steps which also have $\alpha$ as a constituent of their tags. So the discharge in step 3 above was a genuine one, as far as it went. The hypothesis of step 1 lingers on, but the tagged formula in step 4 does not depend on that hypothesis; indeed, in asserting $A \rightarrow A$, we have no need of the hypothesis $A$. (Indeed, in $\mathrm{R}$, we cannot tag step 3 with the $\alpha$ of step 1.) The notation makes this clear; to repeat, only those hypotheses are in force for a given step as are explicitly recorded in the tag on that step.

So the present technique introduces important economies, compared to $[E N T]$. But, in the theory of pure implication, it is not so different, as a

Australasian Journal of Logic (18:5) 2021, Article no. 4 
comparison of [ENT] style proofs of the above theorems of $\mathrm{R}$ will make clear; moreover, the most important economies here could be introduced into [ENT] as well. But, once we are out of the pure theory of implication which the [ENT] technique most directly reflects, the present methods become considerably more flexible. And their eventual import, after all, is conceptual, not mechanical. By basing natural deduction directly on our system, not seriously on a coding of the system, we need not shift, in actually deriving theorems, from one mode of thought to another. The proof of that pudding, I hope, will be in the eating hereafter.

I said above that a "syntactical completeness proof" for the natural deduction system would be superfluous, but let me provide one anyway. Dropping Greek disguise, "statements" of the natural deduction system are of the form $A=B$ or $A \leq B$, where $A, B$ are formulas of $\mathrm{R}$ (or $\mathrm{RQ}, \mathrm{R}^{\sharp}$ as the case may be). Out of Greek disguise, $A=B$ states that $A$ is the same formula as $B$; moreover, such statements only occur in circumstances of tag (Hypothesis) introduction, whence, undisguised, they are all of the form $A=A$. Similarly, $A \leq B$ says that $A \rightarrow B$ is a theorem of the appropriate system; for completeness, we may just as well take $A \leq B$ as a "natural deduction" axiom whenever $A \rightarrow B$ is a theorem. For soundness, we need note only that the rules laid down above do not lead out of the class of $\leq$ statements that correspond to provable $\rightarrow$ statements. (Indeed, as just put, they do not lead out of the class of axioms.) Finally, to assure that all theorems of $R, R Q, R^{\sharp}$ are covered, and not just the $\rightarrow$ formulas, we note that, since $\mathbf{t} \rightarrow A$ is a theorem exactly when $A$ is a theorem, $\mathbf{t} \leq A$ will be a "natural deduction" theorem (indeed, an axiom once more) just when $A$ is an honest theorem of the system in question. Hence our natural deduction system is trivially sound and complete, in a couple of respects, relative to its intended transformation into the axiomatic system.

This merely sums up what was said above while natural deduction was being motivated. But the reader may feel a little uneasy nonetheless. What is the point of having "natural deduction rules," when, if strictly formalized, all the "natural deduction theorems" are already axioms? And again, the point is practical; we desire, if one wants to put it that way, efficient procedures to discover what the natural deduction axioms are (or, really, what the theorems of $\mathrm{R}$ are). In fact, we might always appeal to known inefficient procedures (e.g., recursively enumerating the theorems of $\mathrm{R}$ ) for this purpose; we could even Craig the procedure to reduce the natural deduction axioms to an effective set (saving, for this purpose, the rule of replacement

Australasian Journal of Logic (18:5) 2021, Article no. 4 
for $\wedge$ idempotents). But we don't have time for all that. Hence, natural deduction!

\section{V}

Setting up our natural deduction system has by now occupied the major part of the paper, and it is time that we returned to our principal aim of applying it to $R^{\sharp}$. In order to do so, we now make some further definitions in our language $\mathscr{L}^{\sharp}$.

$$
\begin{aligned}
& D \top . \quad \top \quad={ }_{\mathrm{DF}} \quad 0=0 \\
& D \perp . \perp \quad=_{\mathrm{DF}} \sim \top \quad=_{\mathrm{DF}} \quad 0 \neq 0 \\
& D \nabla . \quad \nabla A \quad={ }_{\mathrm{DF}} \quad \top \wedge A \\
& D \triangle . \quad \triangle A \quad=_{\mathrm{DF}} \quad \perp \vee A \\
& D \equiv . \quad A \equiv B \quad=_{\mathrm{DF}} \quad(A \supset B) \wedge(B \supset A) \\
& D \supset . \quad A \supset B={ }_{\mathrm{DF}} \quad \nabla A \rightarrow B \\
& D \equiv . \quad A \equiv B \quad=_{\mathrm{DF}} \quad(A \supset B) \wedge(B \supset A) \\
& D F . \quad F \quad={ }_{D F} \quad 0=1 \\
& \text { DT. } \mathrm{T} \quad=_{\mathrm{DF}} \sim \mathrm{F} \quad=_{\mathrm{DF}} \quad 0 \neq 1 \\
& D \neg . \quad \neg A \quad={ }_{\mathrm{DF}} \quad A \supset \mathrm{F}
\end{aligned}
$$

We motivate these definitions as follows.

First, $\top, \perp$ will, as suggested above, gradually take over the roles of the sentential constants $\mathbf{t}$ and $\mathbf{f}$. It would be inexpeditious, however, to turn over these roles too suddenly. For $\mathbf{t}$ is a natural deduction convenience, and we have not yet proved that $0=0$ has the right properties to stand in for it. But, once we have proved the necessary theorems, we shall see that $T$ does play the role of an arithmetic least truth, as $\perp$ plays the role of an arithmetic greatest falsehood, whence the need for primitive $\mathbf{t}, \mathbf{f}$ will vanish.

A special role is also played by $\top \wedge A$, relative to $A$, motivating our corresponding definition of $\nabla A$. (Mnemonic: $\nabla$ points down, just like $\top$.) The special role goes back in principle to early work by Ackermann, Anderson, and Belnap, and is made explicit (for $\mathbf{t}$ ) in [IEN]. The idea is to make room for looser notions of follows from than relevantly follows from, without making the notions so loose that we cannot use them to argue relevantly. In such a looser sense, we may use not only $A$ but anything else which we know to

Australasian Journal of Logic (18:5) 2021, Article no. 4 
be true in investigating the consequences of $A$. And since, by $\mathbf{t} \rightarrow$ in a new form $T \rightarrow$, everything that is provable in $\mathrm{R}^{\sharp}$ is going to be provable from our "least truth" $\mathrm{T}$, we see by definition that $\nabla A$ will provably imply not only $A$ but all that, from the $R^{\sharp}$ viewpoint, is known to be true. So the effect of $\nabla$ is to strengthen $A$ so that we may draw from it other conclusions from those to which it is directly relevant.

As $\nabla$ goes, so goes our new defined $\supset$. (Note that this $\supset$ must be kept conceptually and notationally distinct from the material $\supset$.) This $\supset$ is studied in [IEN], where it turns out to have generally intuitionistic properties. (This was already to be expected from Anderson-Belnap [ETH]. Our conception of arithmetic, incidentally, is not intuitionistic, which masks somewhat the underlying logical properties of $\supset$.)

Note the following principles of $R^{\sharp}$.

$$
\begin{array}{ll}
\nabla E . & \nabla A \leq A \\
\nabla I . & A \Rightarrow \nabla A \\
\supset E . & (A \supset B) \wedge \nabla A \leq B \\
\supset I . & A \wedge B \supset C \Leftrightarrow A \supset . B \supset C
\end{array}
$$

As put, $\nabla E$ and $\supset E$ are theorems of $\mathrm{R}^{\sharp}$, while $\nabla I$ and $\supset I$ are rules (the latter a two-sided rule). In view of $\supset E, \supset I, \nabla I$, it is to be noted that, in $\mathrm{R}^{\sharp}$, $\supset$ has the useful properties that modus ponens works for it and that it gives rise to an appropriate deduction theorem relative to ordinary conjunction $\wedge$. (Verification of the latter, however, depends on the left to right direction of $\supset I$, which itself depends on to. So it will not be complete until we have shown that $T$ will serve in $R^{\sharp}$ for $\mathbf{t}$.)

Having $\supset$ in hand cancels in part, within our relevant arithmetic itself, the practical difficulties that might attend to the insistence that we must argue relevantly if we are arguing validly. Such difficulties, e.g., might arise in arguments by cases. For we often dispose, in such arguments, of one case by invoking some relevant hypothesis in force, while another case is settled trivially on background knowledge, without appeal to the hypothesis. But the conjunction of relevant hypothesis and relevant background knowledge settles both cases, and it does so relevantly. Following the line of [ETH] and [IEN], we may encapsulate such appeals to background knowledge in the machinery just introduced.

We have, nevertheless, by no means cancelled relevant implication. For we continue to see the $\rightarrow$ of $R^{\sharp}$, for present purposes, as the underlying 
implication on which arithmetic is being built up. I.e., we find structural arithmetical connections between formulas only in case the corresponding relevant implications or equivalences hold. Such connections must go back to the point that the natural numbers are built up from 0 by adding 1's. Once we begin to see the natural numbers as themselves constituting a universe of discourse, and of certain facts as holding in this universe, we shall indeed wish to express these facts. But there is more than a little mythology in such expression, and so, both for the sake of safety and of clarity, we do not assume that all arithmetical facts are structurally linked. Indeed, this last view is to be seen as an imposition upon arithmetic of certain currently fashionable views in the philosophy of logic - which, however, there are no convincing general reasons to think true. (As to whether there are convincing arithmetical reasons, that's a matter for research. Probably not, but we shall see.)

As $\nabla$ goes, so goes $\Delta$, turning the mnemonic upside down. In view of the DeMorgan laws and definitions, note that $\sim \triangle A$ is provably equivalent to $\nabla \sim A$, etc. And we note, formally, that $\Delta$ has many of the properties normally associated with a possibility operator, while $\nabla$ resembles necessity in the same respects. (E.g., $\nabla E$ above is a theorem, but $\nabla I$ is only a rule.) Other modal analogies, and some disanalogies, will occur to the reader.

Central motivational problems of $\mathrm{R}^{\sharp}$, as yet open, can be related to properties of $\Delta$. (The same holds for relevant logics and theories generally.) For $\Delta$ is related in a simple way to the rule $\gamma$, whose importance for relevant motivation has been apparent ever since Anderson's [OPP]. For $\gamma$ is just the following rule $\supset E$.

\section{$\supset E . \quad A \supset B \& A \Rightarrow B$}

And for $\mathrm{R}^{\sharp}$ (as for relevant logics and theories generally, assuming the presence of the constant $\mathbf{f}$ ), the admissibility of $\gamma$ boils down simply to the admissibility of

$$
\triangle E . \quad \triangle A \Rightarrow A
$$

For a little more practice with our natural deduction system, we shall show that $\supset E$ is admissible in $\mathrm{R}^{\sharp}$ iff $\triangle E$ is. (So as not to clutter up the notation, we shall assume that $\mathbf{t}$ has already been identified with $T$, and $\mathbf{f}$ with $\perp$. We shall make no systematic appeal to the present observation until that has 
been done.)

Suppose, first, that $\supset E$ is admissible in $\mathrm{R}^{\sharp}$. To show $\triangle E$ admissible, let $\triangle A$ be a theorem of $\mathrm{R}^{\sharp}$. We show $A$ a theorem. $\triangle A$ is defined as $\perp \vee A$, which is evidently equivalent to $\top \supset A$. $\top$, of course, is a theorem of $\mathrm{R}^{\sharp}$, whence by $\supset E$ so is $A$.

Suppose conversely that $\triangle E$ is $\mathrm{R}^{\sharp}$-admissible. To show $\supset E$ also admissible, we assume that both $A \supset B, A$ are theorems of $\mathrm{R}^{\sharp}$, and we show $B$ a theorem. Natural deductively, on the parenthetical observation above, we have

$\begin{array}{llll}\text { 1. } & \top \leq & A \supset B & \\ \text { 2. } & \top \leq & A & \\ \text { 3. } & \alpha= & \sim \triangle B & \text { HYPOTHESIS } \\ \text { 4. } & \alpha \leq & \top \wedge \sim B & 3, D \triangle, D \sim \vee, D \top \\ \text { 5. } & \alpha \leq & A \wedge \sim B & 2,4, \text { DiRECTED REPLACEMENT } \\ \text { 6. } & \top \circ \alpha \leq & \perp & 1,5, D \supset, \perp I \text { (i.e., f } I \text { ) } \\ \text { 7. } & \top \leq & \triangle B & 3,6, \sim I\end{array}$

Step 7 means that $\triangle B$ is a theorem of $\mathrm{R}^{\sharp}$, whence by $\triangle E$ so is $B$. This completes the demonstration that one of $\supset E, \triangle E$ is admissible in $\mathrm{R}^{\sharp}$ iff the other one is.

This concludes our natural deduction practice (skipping obvious steps, as we shall henceforth). And, indifferently, we shall henceforth take either $\supset E$ or $\triangle E$ to be the rule $\gamma$ for $\mathrm{R}^{\sharp}$. Question: is $\gamma$ admissible for $\mathrm{R}^{\sharp}$ ?

Answer: I don't know; I suppose so, since $\gamma$ has been shown admissible for an ever wider class of relevant logics and theories; i.e., for RM in [ENT]; for relevant sentential logics in general in [ERG]; for relevant quantification theories in [CRQ]; and for second and higher-order relevant logics in [CR]. In these places, the reader will find general reflection on the significance of $\gamma$ for relevant theories generally; below, we shall reflect in various ways on its significance for $R^{\sharp}$.

Meanwhile, let us note that, even if for some good reason $\gamma$ turns out not to be admissible for $R^{\sharp}$, it might be the case that it is not wanted. If, e.g., $C^{\sharp}$ is inconsistent, $\gamma$ must fail for $\mathrm{R}^{\sharp}$. Fortunately, in that case. Conversely, we shall show that the admissibility of $\gamma$ in $R^{\sharp}$ implies the consistency of $C^{\sharp}$. So, on general Gödelian grounds, a hard proof (if there is one) is to be expected.

Anyway, being prepared for the worst case, we must adjust our proof tech-

Australasian Journal of Logic (18:5) 2021, Article no. 4 
niques in $\mathrm{R}^{\sharp}$ so that they do not assume $\gamma$. We should wish to do this in any case. For $\gamma$, where it holds, we take in general to be a factual principle, not a structural one. And it is just at this point that our hedged semantic faith in classical negation becomes seriously hedged; for inference, we want structural principles, not "facts" about a domain whose entities we are making up as we go along. Technically, this is reflected in the fact that $\gamma$, relevantly speaking, is not a principle which is invariant under assumptions. We shall adjust our technique to take account of this situation.

The contrast between $\supset E$ and $\supset E$ also needs to be noted. The latter, in general, is just a restricted form of $M \rightarrow \wedge$, for the special case in which the $A$ of the latter is of the particular form $\nabla A^{\prime}$. So general $\rightarrow E$ principles may be specialized as appropriate $\supset E$ principles; in that guise, they apply generally to regular R-theories and RQ-theories. Except, however, in a very weak sense (for which cf. [IEN]), we cannot view $\supset E$ as any sort of specialization of $\rightarrow E$. This means that $\supset E$ is admissible only for theories that are semantically normal in a rather strong sense (for which see [ERG] and [SEI]).

Other new connectives and constants can be passed over rather lightly. $\equiv, \equiv$ are respectively the equivalences which go with the implications $\supset, \supset$. They are subject to the same kinds of remarks. F plays the role of an arithmetically absurd proposition; it implies almost (but not quite) everything. Conversely, almost everything implies its negation T. Finally, given that $\supset$ functions as an intuitionist kind of implication, and given that intuitionist negation is absurd (in an [FML] sense), we have in $\neg$ something approaching an intuitionist negation. Thus, there is a sort of intuitionist arithmetic in the $\supset, \neg, \wedge, \vee, \forall X, \exists X$ fragment of $\mathrm{R}^{\sharp}$, built up from the atomic formulas $T=U$ of $R^{\sharp}$. Let us call this fragment $J_{\mathrm{R}}^{\sharp}$.

Note, however, that $J_{\mathrm{R}}^{\sharp}$ is by no means the intuitionist arithmetic $\mathrm{J}^{\sharp}$ (as one finds it, e.g., in $[\mathrm{IMM}]$ ). On two grounds. First, $\mathrm{R}^{\sharp}$ has a classical theory of quantification, which makes $\mathrm{J}_{\mathrm{R}}^{\sharp}$ stronger than $\mathrm{J}^{\sharp}$ on, e.g., certain confinement principles. Second, and more important here, $R^{\sharp}$ does not buy the $J^{\sharp}$ form of the principle that says that 0 is not the successor of any number, on grounds of relevance. For $\mathrm{J}^{\sharp}$ asserts that if $X^{\prime}$ is 0 then all hell breaks loose; i.e., $\neg\left(X^{\prime}=0\right)$ is a theorem of $\mathrm{J}^{\sharp}$; equivalently, $X^{\prime}=0 \supset 0=1$ is a theorem of $\mathrm{J} \sharp$; equivalently, for all $A, X^{\prime}=0 \supset A$ is a theorem of $\mathrm{J}^{\sharp}$.

But these are not theorems of $\mathrm{R}^{\sharp}$, for reasons touched on when motivating the corresponding axiom $A 12$ of $\mathrm{R}^{\sharp}$. For while it is false that 0 is the successor of some number, it is not necessarily absurd. And only, in fact, that kind of 
theory of negation which holds that all falsehoods are absurd could provide general grounds for interpreting the Peano postulate $A 12$ in the $\mathrm{J}^{\sharp}$ way above.

The last point is not to be pushed too far. For we might have specific grounds (e.g., cancellation laws) for preferring the stronger $\mathrm{J}^{\sharp}$ and $\mathrm{C}^{\sharp}$ forms of $A 12$. Here, these specific grounds are not accepted. We accept, indeed, $X^{\prime}=0 \supset 1=0$ as an arithmetical fact, signalled by the material $\supset$. But we do not accept it as an arithmetical law, to be signalled by an $\rightarrow$ or even a $\supset$.

Let us get clear on this point. I am not concerned with the practical inferences which a number theorist will permit himself. So far as practical inferences are concerned, he will no doubt assume that he is working with facts in a numerical universe of discourse, and use $\gamma$. I have no objection to this procedure, if indeed $\gamma$ can be made plausible for this imaginative universe. (I do object, however, to the claim that there is nothing at all problematic about using $\gamma$ in arithmetic. That's, ultimately, an act of faith, and mathematics is not a religion. Or, to the extent that it is one, the worship of Baal becomes plausible by comparison.)

However, what we are concerned with here is the logical framework from which we build up the natural numbers. (It is not ruled out that there are alternative frameworks, good for different purposes.) We are told that there are certain first principles - enunciated by Dedekind and made famous by Peano - on which the natural numbers turn out to be, intuitively and near enough, what vaguely we held them to be. Now certainly this is not the case on first principles alone; unless there are deductive connections between certain number-theoretic statements and others, we shall never get beyond first principles. Moreover, these connections are themselves presupposed in the very vocabulary in which we state the first principles; they are certainly presupposed in the vocabulary in which we formalize these principles.

\section{VI}

Our next topic will be the properties of identity in $R^{\sharp}$. There is at present no commonly accepted relevant theory of identity, even for RQ. Instead, there are alternative relevant theories. Let us briefly say why.

Urquhart has suggested that, at the level of pure predicate calculus with identity, the relevant theory may perhaps be summed up in the following 2 principles.

Australasian Journal of Logic (18:5) 2021, Article no. 4 


$$
\begin{aligned}
& R=. \quad X=X \\
& S={ }^{\star} . \quad Y=Z \rightarrow . A[Y] \rightarrow A[Z], \quad \text { where } A[X] \text { is an atomic formula in which } \\
& X \text { occurs free }
\end{aligned}
$$

One's first thought, rather would have been to have adopted, in place of $S={ }^{\star}$, the more general principle

$$
S=. \quad Y=Z \rightarrow . A[Y] \rightarrow A[Z], \quad \text { where } A[X] \text { is any formula }
$$

However, the latter choice leads to certain apparent anomalies. For, on the general $[\mathrm{IMM}]$ convention that $A[X]$ does not necessarily contain free $X$, we can prove from $S=$ such apparent fallacies of relevance as

(1) $X=Y \rightarrow P \rightarrow P$

(2) $X=Y \rightarrow Z=Z$

in the version of $\mathrm{RQ}$ with identity based on $R=, S=$.

So, it would seem, something along the general lines of the suggested Urquhart approach might better suit relevant logics in general. (Another and apparently even weaker policy was subsequently recommended by Freeman in his contribution to [PRC].) In $\mathrm{R}^{\sharp}$, however, objections to these sorts of theorems do not apply. If one wills, this is because $\mathrm{R}^{\sharp}$ sees its domain of intended application, namely the natural numbers, as already tightly structured, whence we can prove, say, from the assumption that $X=Y$ that $Z=Z$. (There is, however, a catch in this, in that $\mathrm{R}^{\sharp}$ allows for subtraction, in view of the postulate that says that distinct numbers have distinct successors, in a stronger sense than it allows for division. It would be interesting, accordingly, to weaken the subtraction principles, thereby loosening some structural connections and refuting (1) and (2). But I shall not give further consideration to this possibility here.)

Thus given its domain of intended application, the identity theory of $\mathrm{R}^{\sharp}$ will satisfy not only $R=$ and the weak principle $S=^{\star}$, but the stronger principle $S=$. We shall establish that fact in this section, together with the corresponding strong replacement principle for terms. I.e.,

$$
T=. \quad Y=Z \rightarrow . T[Y]=T[Z]
$$

where $T[X]$ is any term and $T[Y], T[Z]$ result respectively from the replace-

Australasian Journal of Logic (18:5) 2021, Article no. 4 
ment of all occurrences of $X$ in $T[X]$ with $Y, Z$.

To begin with, we have, in virtue of axioms $A 13, A 11, A 14$, the following schematic theorems for all terms $T, U$.

$$
\begin{array}{ll}
S U=. & T=U \leq T^{\prime}=U^{\prime} \\
P R=. & T^{\prime}=U^{\prime} \leq T=U \\
S \rightarrow=. & T=U \leq T=V \rightarrow U=V
\end{array}
$$

(Although we have assigned $\leq$ its ordinary arithmetic significance between terms, evidently no ambiguity arises if we continue, as above, to use it between statements for provable implication in $R^{\sharp}$, on our natural deduction practice.)

Evidently these, and subsequent, provable entailments give rise, on directed replacement in $R^{\sharp}$, to 1-premiss natural deduction rules as in IV. We shall indifferently employ, as before, notation introduced to name theorems for the corresponding natural deduction rules as well. We now establish, schematically, some elementary properties.
(1) $\mathbf{t} \leq T+0=T$
$A 15, \forall I, \forall E$
(2) $\mathbf{t} \leq T+0=T \rightarrow T=T$
(1), $S \rightarrow=$, Directed Replacement
(3) $\mathbf{t} \leq T=T$
$(1),(2), \rightarrow E$, to

(3) establishes the reflexivity principle $R=$ for all $T$. Then
(4) $\alpha=\quad T=U$
HYPOTHESIS
(5) $\alpha \leq$
$T=T \rightarrow U=T$
(4), $S \rightarrow=$
(6) $\mathbf{t} \circ \alpha \leq U=T$
(3), (5), $\rightarrow E$
(7) $\mathbf{t} \leq$
$T=U \rightarrow U=T$
(6), (4), $\rightarrow I$

(7) establishes the symmetrical property of identity; i.e.,

$$
S Y M=. \quad T=U \simeq U=T
$$

We henceforth take $S Y M=$ for granted, without explicit comment. It leads almost immediately to the following transitivity rule.

$T R=. \quad \Gamma \leq T=U \& \Delta \leq U=V \Rightarrow \Gamma \circ \Delta \leq U=V$

Australasian Journal of Logic (18:5) 2021, Article no. 4 
The simple verification of $T R=$, using $S \rightarrow=$, is left to the reader. He should note, in particular, the strong mode of tag composition by o in the conclusion of this rule (which may be weakened to $\wedge$ composition by directed (tag) replacement).

Before going further with the properties of $=$, let us return to a favored topic - namely, the elimination of $\mathbf{t}$ from $R^{\sharp}$ in favor of $T$. As a halfway measure, we define

$$
D \top^{\prime} . \quad \top^{\prime}={ }_{\mathrm{DF}} \quad \forall x(x=x)
$$

We shall prove that $T^{\prime}$ has the following crucial property

$$
C P \top^{\prime} . \quad \top^{\prime} \leq A \rightarrow A
$$

for all formulas $A$ of $\mathscr{L}^{\sharp}$ (not containing, of course, the constant $\mathbf{t}$ ).

We prove $C P T^{\prime}$ by induction on the length of $A$. First, suppose that $A$ is an atomic formula $T=U$. Then, evidently,

(8) $T=T \leq T=U \rightarrow T=U \quad S \rightarrow=, S Y M=$

(9) $\quad T^{\prime} \leq T=U \rightarrow T=U \quad D T^{\prime},(8), \forall E$, REPLACEMENT

This disposes of the atomic case. Suppose now, on inductive hypothesis,

(10) $\quad T^{\prime} \leq A \rightarrow A$

(11) $\quad \top^{\prime} \leq B \rightarrow B$

Then

(12) $\quad T^{\prime} \leq \sim A \rightarrow \sim A$

(13) $\quad \top^{\prime} \leq A \wedge B \rightarrow A \wedge B$

(14) $\quad \top^{\prime} \leq A \rightarrow B \rightarrow . A \rightarrow B$

(15) $\quad \top^{\prime} \leq \forall X A \rightarrow \forall X A$

are readily verified on principles set out above, as the reader may check. This disposes of the inductive cases, and establishes $C P \top^{\prime}$ for all formulas $A$ of $\mathscr{L}^{\sharp}$.

Evidently, $T^{\prime} \leq T$, by $\forall E$ and definitions. We wish to show, conversely, that $T \leq T^{\prime}$. For this it will suffice, by $\forall I$, to show

Australasian Journal of Logic (18:5) 2021, Article no. 4 
(16) $0=0 \leq x=x$

We let (16) be our induction proposition $A[x]$, and we use our primitive rule $R M I$ of $\mathrm{R}^{\sharp}$ to prove it. For this we must prove $A[0]$ and $A[x] \rightarrow A\left[x^{\prime}\right]$. But the former is just

(17) $0=0 \leq 0=0$

an instance of $B \rightarrow B$. To prove the latter,

(18) $\alpha=0=0 \rightarrow x=x \quad$ Hypothesis

(19) $\alpha \leq 0=0 \rightarrow x^{\prime}=x^{\prime} \quad(18), S U=$, REPLACEMENT

I.e., $A[x] \leq A\left[x^{\prime}\right]$. Hence we conclude (16) by $R M I$, and

(20) $\quad \top \simeq \top^{\prime}$

in $\mathrm{R}^{\sharp}$ as claimed. In view of (20), we may now transfer the crucial property to $0=0$, inferring

$$
C P \top . \quad \top \leq A \rightarrow A
$$

for all (t-free) formulas of $\mathscr{L}^{\sharp}$. Applying $\rightarrow I$, we get from $C P \top$

(21) $\top \circ A \leq A$

which is half of the wanted version of to. For the other half, it suffices to observe

(22) $\top \circ A \leq \top \circ A$

(23) $\top \leq A \rightarrow \top \circ A \quad(22), \rightarrow I$

whence, since $T$ is a theorem of $R^{\sharp}$ by (essentially) $R=$, we get in $R^{\sharp}$ by $\rightarrow E$ on theorems

$$
\text { (24) } T \leq \top \circ A \quad(23), R=, \rightarrow E
$$

And (21) and (24) together establish the special formal property of a constant $\mathbf{t}$ in relevant theories, namely that it is an identity with respect to o. I.e.,

Australasian Journal of Logic (18:5) 2021, Article no. 4 
T०. $\top \circ A \simeq A$

holds for all (t-free) formulas $A$ of $\mathscr{L}^{\sharp}$. And, since to was the only special property of $\mathbf{t}$ to which we appealed in giving it its general natural deduction role in relevant theories, that role is henceforth assigned in $R^{\sharp}$ to $T$, with all previous principles holding on replacement of $\mathbf{t}$ with $T$.

So $\mathbf{t}$ now departs from this paper. So does $\mathbf{f}$, traded in similarly for $\perp$ on analogy of definition. Officially, $\mathbf{t}, \mathbf{f}$ never belonged to $\mathscr{L}^{\sharp}$ at all, but to a convenient extension thereof, useful for setting up natural deduction. Since $\mathbf{t}$, although it is always contextually definable, is not always eliminable by explicit definition in an arbitrary regular relevant theory, it was good to have it when we were thinking in general terms in IV. But, not being arithmetical, it would become a nuisance for arithmetic itself to retain primitive t. And, as we bid $\mathbf{t}$ farewell, it is important to recall again that we have nowhere proved $\mathbf{t} \simeq T$, even in the extension of $R^{\sharp}$ that contains them both. So the reader is to be pardoned for suspecting that, perhaps, our casual appeal to natural deduction technique somewhere smuggled in an illegitimate appeal to properties of $\mathbf{t}$, and that we have covered our tracks by now expelling $\mathbf{t}$ from the system. Such a suspicious reader is, accordingly, hereby assigned the task of proving To directly in $R^{\sharp}$, without even passing appeals to $\mathbf{t}$. (Hint: we never did appeal to $\mathbf{t}$ to prove To, really.)

That being settled, let us get on with showing that identity has the desired properties $T=, S=$. To begin with, let us vindicate our faith in subtraction by establishing

$$
S B=. \quad X=X \leq 0=0
$$

We prove $S B=$ also by the induction rule $R M I$, taking $X=X \rightarrow \top$ as our induction proposition $A[X]$. Again, $A[0]$ is trivial, and so it suffices to show $A[X] \leq A\left[X^{\prime}\right]$ in $\mathrm{R}^{\sharp}$. So

$$
\begin{array}{ll}
\alpha=X=X \rightarrow \top & \text { Hypothesis } \\
\alpha \leq \quad X^{\prime}=X^{\prime} \rightarrow \top & (25), P R=, \text { Directed Replacement }
\end{array}
$$

(26) is the induction proposition to be demonstrated, whence $S B=$ holds by $R M I$.

$S B=$ can be strengthened, by generalizing on the class of equations. To begin with, every atomic formula $T=U$ counts as a direct equation. A quan- 
tified equation is any result of prefacing zero or more quantifiers (universal or existential) to a direct equation. (Thus, on definitions above, $T \leq U$, $T<U$ are quantified equations.) An indirect equation is either a quantified equation, or is of one of the forms $A \wedge B, A \vee B, A \circ B, \forall X B, \exists X B$, where each of $A, B$ is an indirect equation. (E.g., the law of trichotomy $T<U \vee T=U \vee U<T$ is an indirect equation.) Finally, a secondary equation is any formula $A$ such that, for some indirect equation $B$, it is provable in $\mathrm{R}^{\sharp}$ that $A \rightarrow B$ is a theorem. (Thus, e.g., $T=U \wedge V \neq W$, though not an indirect equation, is a secondary equation, since it provably implies the (indirect) equation $T=U$.)

In view of the transitivity of provable implication, obviously every formula $A$ that provably implies a secondary equation is itself a secondary equation. Classically and intuitionistically, since every formula provably implies $0=0$, every formula would count on the above criterion as a secondary equation. But this, as we shall see, is not the case in $R^{\sharp}$. However, the various classes of equations defined above, of which the secondary equations are the widest class, do play an interesting role in $\mathrm{R}^{\sharp}$. The direct equations and the quantified equations, the latter corresponding intuitively to assertions that certain equations hold universally or have solutions (or have solutions that hold universally, etc.), are of evident special interest. Moreover, the principle $S B=$ generalizes to the whole lot of secondary equations. I.e.,

$=\leq \top . \quad A \leq \top$, whenever $A$ is a secondary equation

In view of the transitivity of provable implication, it suffices to prove $=\leq \top$ for the case in which $A$ is an indirect equation. We do this by induction on the length of $A$. Interesting cases are the atomic case, where $A$ is of the form $T=U$, the case where $A$ is $B \circ C$, and the case where $A$ is $\exists X B$, assuming in the latter two cases that $B$ and $C$ are indirect equations for which $=\leq \top$ has already been established. For the atomic case, note that $T=U \leq T=T$ by symmetry and transitivity of identity, and that $T=T \leq T$ by trivial steps of $\forall I, \forall E, D \top$ in $S B=$, whence by transitivity of provable implication $=\leq \top$ is established for this base case. The inductive cases, including the interesting ones, are left to the reader.

So our standard truth $0=0$, even if not provably implied by absolutely everything, is certainly implied by a whole bunch of formulas. Recalling our definition of $\nabla$, another nice property of secondary equations is that they are unaffected by application of $\nabla$. I.e., 
$=\simeq \nabla . \quad A \simeq \nabla A$, whenever $A$ is a secondary equation

From right to left, this is trivial by $\nabla E$. From left to right, given that $A$ is a secondary equation,

(27) $\alpha=A \quad$ HYPOTHESIS

(28) $\alpha \leq \top \quad(27),=\leq \top$

(29) $A \leq \nabla A \quad(27),(28), \wedge I, D \nabla$

Similarly, when the antecedent of a relevant conditional $A \rightarrow B$ is a secondary equation, $A \rightarrow B$ is completely equivalent to our enthymematic conditional $A \supset B$. I.e.,

$\rightarrow \simeq \supset . \quad A \rightarrow B \simeq A \supset B$, whenever $A$ is a secondary equation

This is immediate from $=\simeq \nabla, D \supset$, and replacement of provable $\mathrm{R}^{\sharp}$ equivalents. So in a large class of cases, including everything in the $\mathrm{J}^{\sharp}$ vocabulary (as that vocabulary is construed relevantly), intuitionist implications are the same as relevant implications. (Again, however, we caution the reader not to think that $\mathrm{J}^{\sharp}$ is thereby a relevant arithmetic, on points noted earlier.)

The last 2 principles pushed $=\leq \top$ in one direction. To push it in another direction, note that

$=\simeq \top . \quad A \simeq \top$, when $A$ is both a secondary equation and a theorem of $\mathrm{R}^{\sharp}$

If we take, imaginatively, provably equivalent formulas in $\mathrm{R}^{\sharp}$ to express the same (arithmetic) proposition, what $=\simeq \top$ amounts to is that very many theorems of $R^{\sharp}$ are going to express the same proposition as the standard truth $0=0$. (By comparison, all provable formulas express the standard truth from the viewpoint of $C^{\sharp}$ and $J^{\sharp}$.) In particular, all direct equations, quantified equations, and conjunctions, disjunctions, and fusions of quantified equations will expressly be $R^{\sharp}$ equivalent to $T$ if they are theorems.

Related principles are the following:

$=\simeq \top^{\star} . \quad A \simeq \top \leftrightarrow A$, whenever $A$ is a secondary equation

$=\leq \top^{\star}$. $\quad A \leq B$, whenever $A$ is a secondary equation and $B$ is a theorem of $\mathrm{R}^{\sharp}$

$\wedge \simeq$ o. $\quad A \circ B \simeq A \wedge B$, whenever both of $A, B$ are secondary equations

Australasian Journal of Logic (18:5) 2021, Article no. 4 
For proof, assuming $A$ a secondary equation,

$$
\begin{array}{lll}
(30) & \alpha=A & \text { Hypothesis } \\
(31) & \alpha \leq \top \rightarrow A & (30), \top \rightarrow \\
(32) & \alpha \leq \top \leftrightarrow A & =\leq \top,(30),(31), \wedge I, D \leftrightarrow \\
(33) & \rho=\top \leftrightarrow A & \text { Hypothesis } \\
(34) & \rho \leq A & (33), D \leftrightarrow, \wedge E, R=, D \top, \rightarrow E \\
(35) & A \simeq \top \leftrightarrow A & (30),(32), \rightarrow I,(33),(34), \rightarrow I, D \leftrightarrow
\end{array}
$$

If also $B$ is a theorem of $\mathrm{R}^{\sharp}, A$ a secondary equation

$$
\begin{aligned}
& \text { (36) } \quad A \leq \top \quad=\leq \top \\
& \text { (37) } T \leq B \quad T T R \\
& \text { (38) } \quad A \leq B \quad(36),(37), \text { REPLACEMENT }
\end{aligned}
$$

If each of $A, B$ are secondary equations,

$$
\begin{aligned}
& \text { (39) } A \wedge B \leq A \circ B \wedge \circ \\
& \text { (40) } \alpha=A \text { Hypothesis } \\
& \text { (41) } \rho=B \quad \text { Hypothesis } \\
& \text { (42) } \rho \leq \quad \top \quad(41),=\leq \top \\
& \text { (43) } \quad \alpha \circ \rho \leq A \quad \text { (40), (42), Т०, } \circ I \\
& \text { (44) } \alpha \circ \rho \leq B \quad \text { In Like Manner To (43) } \\
& \text { (45) } A \circ B \leq A \wedge B \quad \text { (43), (44), } \wedge I, \text {, 40), (41) } \\
& \text { (46) } A \circ B \simeq A \wedge B \quad \text { (39), (45) }
\end{aligned}
$$

These proofs establish the three principles just set out. Note, incidentally, that $=\simeq \top$ follows from $=\simeq \top^{\star}$.

In $R^{\sharp}$, the following three statements are equivalent, for each formula $A$.

(i) $\quad A$ is a secondary equation

(ii) $\quad A \leq \top$

(iii) $A \simeq \nabla A$

For (ii) implies (i) by definition of secondary equation and $D T$, (iii) implies (ii) by $D \nabla, \wedge E$, and (i) implies (iii) by $=\simeq \nabla$.

We note that, in $R^{\sharp}$,

Australasian Journal of Logic (18:5) 2021, Article no. 4 


$$
\begin{aligned}
T \simeq \top . \quad T \simeq \top \wedge \top \simeq \top \vee \top \simeq \top \rightarrow \top \simeq \top \circ \top \simeq \exists X \top \simeq \\
\forall X \top \simeq \top \leftrightarrow \top \simeq \top \supset \top \simeq \nabla \top
\end{aligned}
$$

Accordingly, where $A, B$ are both provable secondary equations of $\mathrm{R}^{\sharp}$, we have by $=\simeq \top$, REPLACEMENT,

$$
\begin{aligned}
=\simeq \top^{\star \star} . \quad \top \simeq A \wedge B \simeq A \vee B \simeq A \rightarrow B \simeq A \circ B \simeq \exists X A \simeq \\
\forall X A \simeq A \leftrightarrow B \simeq A \supset B \simeq \nabla A
\end{aligned}
$$

The considerations just gone through open up a considerable realm in which classical kinds of thinking are going to work in $\mathrm{R}^{\sharp}$. For most purposes, this is fine; cutting loose from the classical intuitions that we have learned to feel at home in, formally, is a bit painful, and it is reassuring that, in many respects, we are still hewing to them. However, we are cutting loose from classical intuition at just those points, relevantly, where it is most suspect. For example, despite our long list of equivalents of $T$ above, it is to be noted that $\top \supset \top$ is not on that list (even though $\top \supset \top$ is on the list). And it is not ruled out that further relevant researches will inhibit some of the moves permitted above.

Relative to the constant $T$, secondary equations play a special role. So it is to be expected that the negations of secondary equations will play a dual role relative to the dual constant $\perp$. By a negated equation, we shall accordingly mean something of the form $\sim(T=U)$, where $T, U$ are terms (i.e., $T \neq U$, on our standard abbreviation). We shall not bother to introduce classes of quantified or indirected negated equations, though this is easy to do on dual analogy with the quantified and indirect equations above. But $A$ shall be a secondary negated equation iff, for some formula $B$ such that $A \leftrightarrow \sim B$ is a theorem of $\mathrm{R}^{\sharp}, B$ is a secondary equation.

Contraposition on principles stated above yield the following

$\perp \leq \neq . \quad \perp \leq A$, whenever $A$ is a secondary negated equation

$\neq \simeq \triangle . \quad A \simeq \triangle A$, whenever $A$ is a secondary negated equation

$\neq \simeq \perp . \quad A \simeq \perp$, whenever $A$ is a secondary negated equation and $\sim A$ is a theorem of $\mathrm{R}^{\sharp}$

$\neq \simeq \perp^{\star} . \quad \sim A \simeq A \leftrightarrow \perp$, whenever $A$ is a secondary negated equation

$\perp \leq \neq^{\star} . \quad B \leq A$, whenever $\sim B$ is a theorem of $\mathrm{R}^{\sharp}$ and $A$ is a secondary negated equation

$\rightarrow \simeq \sim \supset \sim . \quad A \rightarrow B \simeq \sim B \supset \sim A$, whenever $B$ is a secondary negated equation

Australasian Journal of Logic (18:5) 2021, Article no. 4 
$\vee \simeq \sim \rightarrow . \quad A \vee B \simeq \sim A \rightarrow B$, whenever both of $A, B$ are secondary negated equations

$\supset \simeq \rightarrow . \quad A \supset B \simeq A \rightarrow B$, whenever $A$ is a secondary equation and $B$ is a secondary negated equation

Proofs are immediate from definitions, contraposition, and $T$ principles just laid down, and so are left to the reader.

The principle $\perp \leq \neq$ may be taken as the $\mathrm{R}^{\sharp}$ form of the ex falso quodlibet. Note, however, that it is not everything, but just every secondary negated equation, that follows from the false. Indeed, we may take this fact, as above, to characterize the secondary negated equations; i.e., $A$ being any formula, the following are equivalent in $R^{\sharp}$.

(i) $\quad A$ is a secondary negated equation

(ii) $\quad \perp \leq A$

(iii) $A \simeq \triangle A$

Given (ii), it is evident that the class of secondary negated equations, besides containing everything of the form $T \neq U$, is closed under $\wedge, \vee, \circ, \supset$, and prefacing quantifiers; it is even closed under $\supset, \equiv, \equiv$. But note that neither of the classes of secondary equations or secondary negated equations is closed under $\rightarrow$.

We next turn to some relations that obtain, and some which do not obtain, between negated equations and secondary negated equations. The most significant is that we do not have, as a theorem of $R^{\sharp}, \perp \leq T$. We do not want it, of course. But the effect is that equations between terms, in $R^{\sharp}$, are something like modal statements of the form $\square A$, while negated equations are something like statements of possibility. (iii) in the last paragraph, its analogue above, and our previous remarks about $\nabla, \Delta$ convey something of this sense. In [ENT], that possibility is classified as a fallacy of modality. While, in systems based on $R^{\sharp}$, we do not have modal notions (the [ENT] account applying in this context to $\mathrm{E}$ instead), the same kinds of formal intuitions are at work. As relevant formal evidence for the truth of an equation, we should have a hard time counting a negated equation. For the picture is that the equations are the principal facts about the numbers that we are trying to establish; the negated equations are the factual assertions that, if asserted hypothetically, prove untenable in the systematic context. And, while we are willing to go from assertions of untenability in some cases to

Australasian Journal of Logic (18:5) 2021, Article no. 4 
factual claims - we accept double negation, for reasons given above - we see no relevant way of arguing from, say, $2+2 \neq 5$ to $2+2=4$, and believe it reasonable to suppose in general that direct arithmetical facts are not to be established from negations of such direct facts.

Truth-functional and quantificational compounding, it should be noted, does not lead us out of the union of the classes of secondary equations and secondary negated equations. Let us call this union the class of secondary formulas. Note that the class of secondary formulas contains all atomic formulas and is closed under the truth-functional connectives and quantifiers. (In particular, it contains all formulas in the properly classical vocabulary.) Question: are all formulas secondary formulas?

The answer to this question, as we shall see, is "No." It boils down to the question whether $A \rightarrow B$ is a secondary formula whenever each of $A, B$ is a secondary formula. There are four cases, which we may put as follows:

(i) Both of $A, B$ are secondary equations

(ii) Both of $\sim A, B$ are secondary equations

(iii) Both of $A, \sim B$ are secondary equations

(iv) Both of $\sim A, \sim B$ are secondary equations

In case (ii), we may conclude that $A \rightarrow B$ is a secondary equation and hence a secondary formula. (For, since the class of secondary equations is closed under $\vee, \sim A \vee B$ is a secondary equation. So by $D \supset,=\leq \top, A \supset B \leq \top$. But $A \rightarrow B \leq A \supset B$ by $R \rightarrow \supset$, whence $A \rightarrow B \leq \top$.) In case (iii), we may conclude that $A \rightarrow B$ is a secondary negated equation and hence a secondary formula. (For $\perp \leq B$ by $\perp \leq \neq$. By $\top \rightarrow$, we have $\perp \leq \top \rightarrow B$. By $=\leq \top$, applicable because $A$ is a secondary equation, we have $\perp \leq A \rightarrow B$ on a use of directed replacement.)

The cases (i) and (iv) march together, in view of contraposition. But they do not march anywhere in particular, so far as the present problem is concerned. Below, in fact, we shall find formulas $A, B$ which are both secondary equations, but such that $A \rightarrow B$ is not a secondary formula. For now, we merely observe that not all formulas are secondary formulas.

Let us return now to the concerns with which we opened this section. We began by attempting to fit the identity theory of $R^{\sharp}$ in particular into a general relevant framework. The actual logical content of the identity axioms of $R^{\sharp}$ strikes one as quite weak; we appealed to that content to show, rather trivially, that identity in $\mathrm{R}^{\sharp}$ has the expected properties of reflexivity, 
symmetry, and transitivity. But identity functions in $\mathrm{R}^{\sharp}$ not merely as part of a general logical theory, but, more important, as part of our arithmetic. So arithmetical interconnections, as seen from $R^{\sharp}$, will be important to establish even general logical laws for identity, above and beyond those which we can all agree hold on fundamental principles of relevant logic. Our constant $T$, the foundation law $0=0$ of arithmetic, has significant properties, which we have here surveyed, in terms of which the proof theory of $R^{\sharp}$ in general, and of its identity theory in particular, will henceforth be developed.

\section{VII}

In this section, we wish to do some actual formal arithmetic, from the $\mathrm{R}^{\sharp}$ point of view. To begin with, let us think about our natural deduction system once again.

First, we are going to make some notational changes in it. While, thus far, we have been writing things like

\section{(1) $\alpha=A$ HyротHesis}

we switch this notation to

$\alpha \quad(1) \quad A \quad$ Hypothesis

Similarly, where we might previously have gone from step (1) to

$$
\text { (2) } \quad \alpha \leq A \vee B \quad(1), \vee I
$$

this will be, in our new notation

$$
\alpha \quad(2) \quad A \vee B \quad(1), \vee I
$$

For its interpretation, the new notation is to be translated directly back into the old notation and interpreted as before. (Note, for this translation, that a tag is an $\alpha=$ tag iff the step which it tags bears the annotation Hypothesis; otherwise, it is an $\alpha \leq$ tag.) Tags, however, firmly remain formulas, provably implying the formulas they tag, and we shall continue to switch easily back and forth between Greek and Latin notation, a switch justified by our Hypothesis steps, and even to use Latin notation in tags.

Australasian Journal of Logic (18:5) 2021, Article no. 4 
Where convenient, we shall also use the old notation for tagged formulas. (E.g., when we wish to call psychological attention to the $\mathrm{R}^{\sharp}$ implication that is provable, instead of to the tagged formula which is provable under some hypotheses.)

Particularly important, so far as tagging formulas is concerned, are considerations that rest on the principle $=\leq T$ of the last section, on which every secondary equation provably implies $T$. This means that, whenever a tag $\Gamma$ is a secondary equation, and when $A$ is known to be a theorem of $\mathrm{R}^{\sharp}$, we are entitled to a step, say

\section{$\Gamma \quad(3) \quad A$,}

in virtue of $T \leq A$ and directed replacement. In fact, we shall very often be concerned with hypotheses that are secondary equations. And our principal tag compounding maneuvers, under $\wedge, \vee, \circ, \forall X, \exists X$, do not lead out of the class of secondary equations, when applied to tags that are in this class. So steps like (3) can occur rather frequently. Caution: such steps remain illegitimate when $\Gamma$ is not a secondary equation, even if $A$ is a theorem of $\mathrm{R}^{\sharp}$ anyway.

A last notational simplification. In tags, we shall henceforth drop $\circ$ in favor of simple juxtaposition. (And we modify our general notational conventions to let juxtaposition bind even more tightly than $\wedge$. However, to keep the number of parentheses down, an explicit notational occurrence of $\circ$ can still be indulged in, binding less tightly than $\wedge$ as before.) This is quite important, because $\circ$, the tag combiner which is introduced by $\rightarrow E$ and other important 2 premiss rules, occurs constantly; so the reader should remember that it is still there, even if invisibly. (On our general formal philosophy, naturally, all the connectives are there invisibly, but $\circ$ will henceforth be a little more invisible than most.)

Henceforth, our appeal to the rule $R M I$ of mathematical induction has been minimal. It is, however, a principal tool for arithmetical deduction, and so we wish to bring it under our general natural deduction technique.

First, let us think about a direct application of $R M I$. This is an inference from $A[0]$ and $A[X] \rightarrow A\left[X^{\prime}\right]$, once we have proved them in $\mathrm{R}^{\sharp}$, to $A[X]$. Dressed up, in our natural deduction format, the inference will henceforth look something like this.

T (4) $A[0] \quad$ JustificAtion

Australasian Journal of Logic (18:5) 2021, Article no. 4 


$\begin{array}{llll}\alpha & (5) & A[X] & \text { Hypothesis } \\ & \vdots & \vdots & \\ \alpha & (71) & A\left[X^{\prime}\right] & \text { JustifiCATiON } \\ \top & (72) & A[X] & (4),(5),(71), R M I\end{array}$

Often, however, we shall wish to carry out inductive arguments under hypotheses (including hypotheses entertained for some outer inductive argument within which we are nesting an inner inductive argument). So it is important to get straight on the kinds of inductive argument that are invariant under assumptions. (It is important, moreover, that we have for this purpose mathematical induction in the "theorem" form A19 of Section II.)

So suppose that we are given

$\begin{array}{llll}\Gamma & (6) & A[0] & \text { JustifiCATion } \\ \Delta & (7) & \forall X\left(A[X] \rightarrow A\left[X^{\prime}\right]\right) & \text { JustificAtion }\end{array}$

Then, by an application of $\wedge I$, we have
$\Gamma \wedge \Delta$
(8)
$A[0] \wedge \forall X\left(A[X] \rightarrow A\left[X^{\prime}\right]\right)$
$(6),(7), \wedge I$

whence, by directed replacement and $A 19$, we may conclude

$$
\Gamma \wedge \Delta \quad(9) \quad \forall X A[X]
$$

So we may take (6) and (7) directly as premisses from which (9) may be derived, tagged as noted, and justified by a notation $R M I$ which we shall henceforth consider all-purpose, so far as inductive arguments are concerned.

(6), normally, will have to be justified directly. But (7) tempts us to further analysis. For we should normally prove (7) by assuming $A[X]$, deriving $A\left[X^{\prime}\right]$ relevantly on that on that hypothesis, and then generalizing with respect to $X$. So, on further refinement, we proceed

$$
\begin{array}{llll}
\alpha & (0) & A[X] & \text { Hypothesis } \\
\Delta \circ \alpha & (00) & A\left[X^{\prime}\right] & \text { JustificAtion }
\end{array}
$$

whence, provided that we have safeguarded the $\forall I$ step envisioned above by insisting that $X$ should not occur free in $\Delta$, we may infer (9) once more, citing this time (0), (00), (6), RMI as justification. And this, in fact, will 
be our preferred form of $R M I$.

Let us, now, clean up the above discussion slightly. Ordinarily, there will be little point in making $\Gamma, \Delta$ distinct tags. So suppose that $\Gamma$ is a tag in which $X$ does not occur free. (In practice, the reader will recall, this means that $X$ must not occur free in any of the hypotheses which components of $\Gamma$ are introduced to tag.) Then the general pattern of an inference by $R M I$ will usually look like this.

$\begin{array}{llll}\Gamma & (6) & A[0] & \text { JUstifiCAtion } \\ & \vdots & \vdots & \\ \alpha & (10) & A[X] & \text { Hypothesis } \\ & \vdots & \vdots & \\ \Gamma \alpha & (73) & A\left[X^{\prime}\right] & \text { JUstifiCATION } \\ \Gamma & (74) & A[X] & (6),(10),(73), R M I\end{array}$

Two further points deserve minor note. First, the "direct application" of $R M I$ which was schematized in introductory remarks is just the special case of the above rubric in which $\Gamma$ is taken as $T$. (In this special case, the tag $\Gamma \alpha$ on (73) is completely equivalent to the tag $\alpha$ on (71), since $\top \alpha$ is equivalent to $\alpha$ by Т०.)

The second minor point is that, in stating the conclusion (74), we left off the prefaced $\forall X$ which we had previously attached to (9). Since it is important for the rubric that $X$ not occur free in $\Gamma$, this is an indifferent point, since the quantifier may be immediately restored by an application of $\forall I$. In fact, we are going to be henceforth rather cavalier about trivial application of rules like $\forall I, \forall E$. When the conditions for their application are met, we shall merely apply them, without necessarily taking specific notice of that fact. (In fact, we have been a bit cavalier already.) Consider, e.g., the principle $R=$. We might take this principle to be any of the following: $x=x$, $\forall x(x=x), X=X, \forall X(X=X), T=T$. The first two are specific theorems of $\mathrm{R}^{\sharp}$; the third and fourth are theorem schemes for an unspecified variable $X$; and the last is a theorem scheme for an unspecified term $T$, which may be (but which need not be) a variable. However, all of these schemes translate into each other by trivial steps of $\forall I, \forall E$ (bearing in mind, in the final three cases, that schemes are assumed for all their instances).

So we allow ourselves, henceforth, such minor variations on the way that we put our principles, and the trivial transformations which turn one such

Australasian Journal of Logic (18:5) 2021, Article no. 4 
variant into another. And we shall similarly presume on the reader's good will in other minor ways; e.g., in picking bound variables fresh to context when necessary to avoid confusion of bound variables. (Example: $D \leq$ above.)

So much for trivia. Let us return to induction. $A 19$ above, on which we depended for the natural deduction story told thus far about $R M I$, was stated in terms of relevant implication $\rightarrow$. But it might just as well have been stated in terms of our enthymematic implication $\supset$. I.e., the following is a theorem scheme of $R^{\sharp}$.

$$
A 19^{\star} \quad A[0] \wedge \forall X\left(A[X] \supset A\left[X^{\prime}\right]\right) \supset \forall X A[X]
$$

Let us, in fact, prove $A 19^{\star}$.

$\begin{array}{llll}\alpha & (11) & \nabla\left(A[0] \wedge \forall X\left(A[X] \supset A\left[X^{\prime}\right]\right)\right) & \text { Hypothesis } \\ \alpha & (12) & \top \wedge A[0] & (11), D \nabla, A \wedge, \wedge E \\ \rho & (13) & \top \wedge A[X] & \text { Hypothesis } \\ \alpha & (14) & \top \wedge A[X] \rightarrow A\left[X^{\prime}\right] & (11), \wedge E, D \supset, D \nabla \\ \alpha \rho & (15) & A\left[X^{\prime}\right] & (13),(14), \rightarrow E \\ \alpha \rho & (16) & \top & (12), \wedge E,(13), \wedge E, \circ I, \top \circ \\ \alpha \rho & (17) & \top \wedge A\left[X^{\prime}\right] & (15),(16), \wedge I \\ \alpha & (18) & \top \wedge A[X] & (12),(13),(17), R M I \\ \alpha & (19) & A[X] & (18), \wedge E \\ \top & (20) & A 19^{\star} & (11),(19), D \supset, \rightarrow I, \forall I\end{array}$

Note that, in the application of $R M I$ completed in step (18), the induction proposition is not simply $A[X]$. Instead, by definition, it is $\nabla A[X]$.

Having proved $A 19^{\star}$, note that the form of mathematical induction which was denied us in the $\rightarrow$ case (for all we know, anyway) is O.K. in the $\supset$ case. For, while $A 19^{\prime}$ in Section II could not be justified there, in the intuitionistically acceptable atmosphere of $A 19^{\star}$ we may move directly, on R-valid principles for defined $\supset$, to

$$
A 19^{\star \prime} \quad \forall X\left(A[X] \supset A\left[X^{\prime}\right]\right) \supset . A[0] \supset A[X]
$$

Having $A 19^{\star}$ allows us to weaken our opening direct scheme for proving theorems of $\mathrm{R}^{\sharp}$ by mathematical induction. For this scheme can now be put 


$\begin{array}{llll}\top & (4) & A[0] & \text { JustificAtion } \\ \alpha & \left(5^{\prime}\right) & \nabla A[X] & \text { Hypothesis } \\ & \vdots & \vdots & \\ \alpha & (71) & A\left[X^{\prime}\right] & \text { JustifiCATION } \\ \top & (72) & A[X] & (4),(5),(71), R M I\end{array}$

I.e., we are allowed to conjoin in $\top$ to the hypothesis $A[X]$ of the inductive stage $\left(5^{\prime}\right)$ of our inductive argument, carrying everything else as before. This gives us a stronger hypothesis, whose effect is to allow us to import our background knowledge into our inductive argument from $A[X]$ to $A\left[X^{\prime}\right]$. (Note, however, that this strengthening is illusory when $A[X]$ is already an equation or secondary equation, since in that case it provably implies $\nabla A[X]$ anyway by $=\simeq \nabla$.)

Using $A 19^{\star}$ in a way that is invariant under natural deduction assumptions, on the other hand, requires a bit of care. (The main point is that, in justifying the last rubric formally, we would have to appeal to the admissibility of the rule $\nabla I$. While the rule is admissible, for theorems of $\mathrm{R}^{\sharp}$, it is not invariant under assumptions. So we must take care.)

Let us introduce, accordingly, $\nabla \Gamma$ for a tag that is (as a formula) a secondary equation. (E.g., any tag $\Gamma$ which implies $\top$, and hence is equivalent to $\nabla \Gamma$, will fall schematically under this rubric. Similarly, when we know that a tag is, as a formula, a secondary equation, we shall move without further notational notice trivially between $\Gamma$ and $\nabla \Gamma$.)

Under these circumstances, the general rubric above may be put, where $X$ does not occur free in $\nabla \Gamma$,

$\begin{array}{llll}\nabla \Gamma & \left(6^{\prime}\right) & A[0] & \text { Justification } \\ & \vdots & \vdots & \\ \nabla \alpha & \left(10^{\prime}\right) & \nabla A[X] & \text { Hypothesis } \\ & \vdots & \vdots & \\ \nabla \Gamma \wedge \nabla \alpha & \left(73^{\prime}\right) & A\left[X^{\prime}\right] & \text { JustificAtion } \\ \nabla \Gamma & \left(74^{\prime}\right) & A[X] & \left(6^{\prime}\right),\left(10^{\prime}\right),\left(73^{\prime}\right), R M I\end{array}$

Now let us return to our view of $\nabla$ as a defined operation on formulas, rather than as indicative of a special category of tags. $\nabla$ is interesting to us on account of three properties: First, the $\nabla E$ property reflecting the R- 
theoremhood of $\nabla A \rightarrow A$; second, the $\nabla I$ property reflecting the R-admissible rule $A \Rightarrow \nabla A$; third, the property, inherited from $\top \rightarrow$, that makes $B \rightarrow$. $\nabla A \rightarrow B$ a theorem of $\mathrm{R}^{\sharp}$, and which makes $\nabla A \circ \nabla B$ provably equivalent to $\nabla A \wedge \nabla B$. On the first of these properties, a step of replacement always permits us to retag $\Gamma$ as $\nabla \Gamma$ on the "tagged formula" $A$, even if $\Gamma$ is not a secondary equation. On the third, we can always tag with $\nabla \Gamma$ anything tagged with $T$, for any $\Gamma$ whatsoever; and we can always infer from a tagged formula $\nabla A$ any theorem of $\mathrm{R}^{\sharp}$, with the same tag. Moreover, we can trade $\nabla$ tags compounded by $\wedge$ for the same tags compounded by $\circ$ (e.g., with a subsequent step of $\rightarrow I$ in mind).

All these things having been said, let us put the above rubric in the strongest useful form.

$\begin{array}{llll}\Gamma & \left(6^{\star}\right) & A[0] & \text { JustificAtion } \\ & \vdots & \vdots & \\ \alpha & \left(10^{\star}\right) & \nabla A[X] & \text { Hypothesis } \\ & \vdots & \vdots & \\ \Gamma \wedge \alpha & \left(73^{\star}\right) & A\left[X^{\prime}\right] & \text { JustificAtion } \\ \nabla \Gamma & \left(74^{\star}\right) & A[X] & \left(6^{\star}\right),\left(10^{\star}\right),\left(73^{\star}\right), R M I\end{array}$

As usual, $X$ must not occur free in $\Gamma$. And, we note, the above rubric is particularly useful because we allow ourselves the strong inductive hypothesis $\left(10^{\star}\right)$, while we need only derive the conclusion $\left(73^{\star}\right)$ with the weak tag $\Gamma \wedge \alpha$. This combination of strong hypothesis and weak conclusion, so far as the inductive stage of an inductive argument is concerned, makes this form of mathematical induction particularly easy to work with. But there is no help for the fact, in general, that the tag on the ultimate conclusion of the argument, namely $\left(74^{\star}\right)$, must be of the form $\nabla \Gamma$. Often, we don't care about this, either because $\Gamma$ is itself a secondary equation or because we are aiming at an eventual conclusion tagged with $T$, to which a conclusion tagged with $\nabla T$ is the same thing. But, whenever it is important that we retain the tag $\Gamma$, and do not weaken it to $\nabla \Gamma$, then the last rubric won't do, and we must fall back on the properly relevant rubrics set out above.

Australasian Journal of Logic (18:5) 2021, Article no. 4 


\section{VIII}

Now let us do some arithmetic. We shall busy ourselves, mainly, with demonstrations that the relevant arithmetic $\mathrm{R}^{\sharp}$ has, roughly, the same substantive theorems as anybody's arithmetic. In doing so, we have Kleene's [IMM] particularly in mind, adapting Kleene's proofs straightforwardly in many cases. Two points, however, require care. First, despite the fact that it is often held that nobody really reasons from contradictions to arbitrary formulas, [IMM] in fact does so quite often. We neither wish, nor shall attempt, to incorporate such fallacious arguments here. (The reader may spot these arguments in [IMM] by looking for references to weak $\neg$ elimination; I take it that what is weak about such moves is that there is a weak case that they are valid, since the logical principle on which they rest is so strong that, to the extent to which formalization is supposed to represent and to codify intuitively acceptable mathematical argument, [IMM] fails to do so on this point.) So important modification of many [IMM] proofs is in order.

Second, to repeat a point touched on earlier, we must concern ourselves not only with the proofs but with the formal statement of the theorems of intuitive mathematics. Once we have got rid of the notion that there is a canonical language, embodying canonical principles of inference, in which this can be done, we are returned to a more innocent state in which we are permitted to wonder, "What is formal arithmetic?" And, once we have got used to the further notion that formal methods need not merely reflect, or be an instrument in the reconstruction of, our naive mathematical intuitions, but may be used to shape these intuitions, then our formal homework appears in a new light. In that light, it is no longer tolerable to hold that we are just "formalizing" some necessary truths with which we are already thoroughly familiar, or the body of which, at the very least, have already been "characterized" by some existing postulate set (e.g., the Dedekind-Peano one). For there is no such characterization of the arithmetical truths without a companion characterization of the arithmetically acceptable inferences, and indeed of the arithmetical language. It is not enough that we should agree on such things, that eminent authority should recommend them to us, or that platonic myths should have been invented for their support. To understand, we must begin again, as ever, at the beginning, and work things out ourselves, from the beginning. We have, to be sure, certain criteria as to what we expect to do, and hope to do, themselves grounded in the existing practices which stem from the critical investigations into the foundations of 
mathematics initiated in the last century. We expect, in fact, that the results of our inquiry will be conservative relative to these investigations. But, given the confusion into which the foundations of mathematics were plunged by Russellian and Gödelian anomalies, and the ever more scholastic character of the enterprises that have arisen from the attempt to dispel these anomalies, beginning again at the beginning may not be a half-bad idea. For what we need to do is to get a firmer feel for formal methods as such, without committing ourselves too soon to particular formal methods that will suffer forever from the philosophical limitations of the viewpoint from which they have sprung.

As a contribution to such great enterprises, what is done here is severely pedestrian, since we shall concentrate on the most elementary properties of and functions on the natural numbers. Since, for now, even the stability of $R^{\sharp}$ is in doubt, and given the conservative character of our present aims and methods, the Big Questions will have to wait. Not only arithmetic, but all of mathematics awaits relevant reconstruction. And, in the end, it is the Big Questions that must be faced. The import of all formal work on foundational questions is, in the last analysis, philosophical, and it misses its purpose when it simply strays down arcane technical by-ways. If the set myth won't do, if a correspondence theory of mathematical truth won't do, if an empty tautological justification of mathematics won't do, then much of the arcane wandering has already missed its purpose. We are back, indeed, with Dedekind, Frege, and Cantor, confronting their questions, but not content with their answers. So, proceeding sometimes as they did, and sometimes perhaps breaking with them, we shall have to answer their questions afresh, perhaps in quite different ways.

Dedekind, Frege, and Cantor, we suppose, thought that ordinary addition was commutative. So do we. Let us see what other points of elementary agreement we can find.

First, let us develop some of the elementary properties of + . Among them are

+0. $\quad X+0=X$

0+. $0+X=X$

$+1 . \quad X+1=X^{\prime}$

+2. $X+2=X^{\prime \prime}$

$\vdots \quad \vdots$

Australasian Journal of Logic (18:5) 2021, Article no. 4 


$$
\begin{array}{ll}
A+. & X+Y+Z=X+(Y+Z) \\
C+. & X+Y=Y+X \\
C A+. & X+Y+Z=X+Z+Y \\
M+. & X=Y \rightarrow X+Z=Y+Z \\
N+. & X=Y \rightarrow Z+X=Z+Y \\
M-. & X+Z=Y+Z \rightarrow X=Y
\end{array}
$$

The principle +0 is our axiom $A 15$. The other principles we shall have to prove. Let us begin with $0+$.

$\begin{array}{llll}\top & (1) & 0+0=0 & +0 \\ \alpha & (2) & 0+X=X & \text { Hypothesis } \\ \alpha & (3) & 0+X^{\prime}=X^{\prime} & (2), S U=, A 16, T R= \\ \top & (4) & 0+X=X & (1),(2),(3), R M I\end{array}$

Similarly trivial are $+1,+2, \ldots$ E.g.,

$$
\begin{gathered}
\top \quad(5) \quad X+1=X+0^{\prime}=(X+0)^{\prime}=X^{\prime} \quad \begin{array}{l}
D 1, \quad A 16, \quad T R=, \quad+0, \quad S U=, \\
T R=
\end{array}
\end{gathered}
$$

When convenient, we use abbreviated notation as in (5) for simple arguments by $T R=$, not necessarily so annotated. Let us move on to $M+$.

$\begin{array}{llll}\alpha & (6) & X=Y & \text { Hypothesis } \\ \alpha & (7) & X+0=Y+0 & (6),+0, T R= \\ \rho & (8) & X+Z=Y+Z & \text { Hypothesis } \\ \rho & (9) & X+Z^{\prime}=Y+Z^{\prime} & (8), S U=, A 16, T R= \\ \alpha \wedge \rho & (10) & X+Z^{\prime}=Y+Z^{\prime} & (9), \wedge E, \text { TAG REPLACEMENT } \\ \nabla \alpha & (11) & X+Z=Y+Z & (7),(8),(10), R M I,=\simeq \nabla \\ \top & (12) & X=Y \rightarrow X+Z=Y+Z & (8),(11),=\simeq \nabla, \rightarrow I\end{array}$

We reached step (11) by induction on $Z$, using the form of $R M I$ considered last in VII (depending on $A 19^{\star}$ ). This entitled us to a prefaced $\nabla$ on step (8), which we ignored as superfluous since (8) is a (secondary) equation. Note also that, since the conclusion (9) depends only on (8) (and not also on (6)) we conjoined in the tag $\alpha$ on (6) by $\wedge E$ to get step (10). Since it is ordinary conjunction $\wedge$, not fusion $\circ$, by which we compound tags in this

Australasian Journal of Logic (18:5) 2021, Article no. 4 
form of $R M I$, no questions of relevant use arise and the step (10) is wellmotivated. We are unconcerned that the form of $R M I$ used here results in the tag $\nabla \alpha$ in step (11). For $\alpha$ itself is also a (secondary) equation, whence $\nabla$ is removed to make the tags on (11), (6) match, allowing the final step (12) by $\rightarrow I$. We shall not be so explicit in the future, nor need the reader be constantly alert to these subtle moves. He must, however, keep in mind the motivational principles set out above on which they rest. For otherwise he might come to think that moves buried in annotations (or passed over tacitly) are superfluous, leading perhaps to the suspicion that we are making moves which we are not making, and which it would be motivationally incorrect, and destructive of claims to relevant insight, to make. In short, while none but the most careful readers will want to check the proofs to come in formal detail, writing out a few proofs in full is always useful to get the hang of what is going on, other than those which the author has made reasonably complete, and so the reader should be alert to just the kinds of moves made explicit above, and to reasons already provided for them.

$M+$ shows, in one sense, that we can add sensibly in $\mathrm{R}^{\sharp}$; in the same sense, $M$ - shows that we can subtract. Here we use the original direct form of $R M I$ for induction on $Z$.

$\begin{array}{llll}\top & (13) & X+0=Y+0 \rightarrow X=Y & +0, T R= \\ \alpha & (14) \quad X+Z=Y+Z \rightarrow X=Y & \text { Hчротнеsis } \\ \rho & (15) \quad X+Z^{\prime}=Y+Z^{\prime} & \text { Hyротнеsis } \\ \rho & (16) & X+Z=Y+Z & (15), A 16, T R=, P R= \\ \alpha \rho & (17) \quad X=Y & (14),(16), \rightarrow E \\ \alpha & (18) \quad X+Z^{\prime}=Y+Z^{\prime} \rightarrow X=Y & (15),(17) \rightarrow I \\ \top & (19) \quad X+Z=Y+Z \rightarrow X=Y & (13),(14),(18), \rightarrow I\end{array}$

Before proving $C+$, it is helpful to prove the following.

$\top \quad(20) \quad X^{\prime}+Y=X+Y^{\prime}$

We prove (20) thus.

$\begin{array}{llll}\top & (21) & X^{\prime}+0=X+0^{\prime} & +0,+1, D 1, T R= \\ \alpha & (22) & X^{\prime}+Y=X+Y^{\prime} & \text { Hypothesis } \\ \alpha & (23) & X^{\prime}+Y^{\prime}=X+Y^{\prime \prime} & (22), S U=, A 16, T R=\end{array}$

Australasian Journal of Logic (18:5) 2021, Article no. 4 
(20) now follows from $(21),(22),(23)$ by the direct form of $R M I$. Now let us prove $C+$.

$$
\begin{array}{llll}
\top & (24) & 0+Y=Y+0 & 0+,+0, T R=, \forall I \\
\alpha & (25) & X+Y=Y+X & \text { Hypothesis } \\
\alpha & (26) & X+Y^{\prime}=(X+Y)^{\prime}=(Y+X)^{\prime}= & (25), S U=, A 16, T R= \\
& & Y+X^{\prime} & \\
\alpha & (27) & X^{\prime}+Y=Y+X^{\prime} & (20),(26), T R= \\
\top & (28) & X+Y=Y+X & (24),(25),(27), R M I
\end{array}
$$

We may now add the left monotony principle $N+$ to the right monotony principle $M+$ already proved.
$\alpha \quad(29) \quad X=Y$
HYPOTHESIS
$\alpha \quad(30) \quad X+Z=Y+Z$
(29), $M+$
$\alpha \quad(31) \quad Z+X=Z+Y$
(30), $C+, T R=$
$\top \quad(32) \quad X=Y \rightarrow Z+X=Z+Y$
(29), (31), $\rightarrow I$

We next prove the associative principle $A+$.

$$
\begin{array}{llll}
\top & (33) & X+Y+0=X+(Y+0) & +0, N+,+0, T R= \\
\alpha & (34) & X+Y+Z=X+(Y+Z) & \text { Hypothesis } \\
\alpha & (35) & X+Y+Z^{\prime}=X+(Y+Z)^{\prime} & (34), S U=, A 16, T R= \\
\top & (36) & (Y+Z)^{\prime}=Y+Z^{\prime} & A 16 \\
\top & (37) & X+(Y+Z)^{\prime}=X+\left(Y+Z^{\prime}\right) & (36), N+ \\
\alpha & (38) & X+Y+Z^{\prime}=X+\left(Y+Z^{\prime}\right) & (35),(37), T R= \\
\top & (39) & X+Y+Z=X+(Y+Z) & (33),(34),(38), R M I
\end{array}
$$

$C A+$, which combines the commutative and associative laws, is easily proved from $C+$ and $A+$, completing our verification of the most elementary principles of addition. Next, naturally, is multiplication, together with principles relating addition and multiplication.

$$
\begin{array}{ll}
\times 0 . & X \times 0=0 \\
0 \times . & 0 \times X=0 \\
\times 1 . & X \times 1=X \\
\times 2 . & X \times 2=X+X
\end{array}
$$

Australasian Journal of Logic (18:5) 2021, Article no. 4 


$\begin{array}{ll}\vdots & \vdots \\ A \times . & X \times Y \times Z=X \times(Y \times Z) \\ C \times . & X \times Y=Y \times X \\ C A \times . & X \times Y \times Z=X \times Z \times Y \\ M \times . & X=Y \rightarrow X \times Z=Y \times Z \\ N \times . & X=Y \rightarrow Z \times X=Z \times Y \\ D \times+. & X \times(Y+Z)=X \times Y+X \times Z\end{array}$

The principle $\times 0$ is our axiom $A 17$. In proving other principles, we may take the properties of + already established for granted. We begin with $0 \times$ :

$$
\begin{array}{llll}
\top & (40) & 0 \times 0=0 & \times 0 \\
\alpha & (41) & 0 \times X=0 & \text { Hypothesis } \\
\alpha & (42) & 0 \times X^{\prime}=0 \times X+0=0 \times X=0 & A 18,+0, T R=,(41), T R= \\
\top & (43) & 0 \times X=0 & (40),(41),(42), R M I \\
\times 1, \times 2, \ldots & \text { come next. } & \\
\top \quad(44) & X \times 1=X \times 0^{\prime}=X \times 0+X= & D 1, A 18, \times 0, M+, 0+ \\
& & 0+X=X & \\
\top & (45) & X \times 2=X \times 1^{\prime}=X \times 1+X=X+X & D 2, A 18, \times 1, M+
\end{array}
$$

And so forth. $M \times$ is also straightforward.

$\begin{array}{llll}\alpha & (46) & X=Y & \text { Hypothesis } \\ \alpha & (47) & X \times 0=0=Y \times 0 & \times 0, T R=,=\leq \top,(46) \\ \rho & (48) & X \times Z=Y \times Z & \text { Hypothesis } \\ \rho & (49) & X \times Z+X=Y \times Z+X & (48), M+ \\ \alpha & (50) & X \times Z+X=Y \times Z+Y & (46), N+ \\ \alpha \rho & (51) & X \times Z+X=Y \times Z+Y & (49),(50), T R= \\ \alpha \rho & (52) & X \times Z^{\prime}=Y \times Z^{\prime} & (51), A 18, T R= \\ \alpha & (53) & X \times Z=Y \times Z & (47),(48),(52), R M I \\ \top & (54) & X=Y \rightarrow X \times Z=Y \times Z & (46),(53), \rightarrow I\end{array}$

Note that the above argument uses the form of $R M I$ that rests in $A 19$. To prove $C \times$ it is helpful to prove

Australasian Journal of Logic (18:5) 2021, Article no. 4 
$\top \quad(55) \quad X^{\prime} \times Y=X \times Y+Y$

We prove (55) thus.

$$
\begin{array}{llll}
\top & (56) & X^{\prime} \times 0=0=X \times 0+0 & \times 0,+0, M+ \\
\alpha & (57) & X^{\prime} \times Y=X \times Y+Y & \text { HYPOTHESIS } \\
\top & (58) & X^{\prime} \times Y^{\prime}=X^{\prime} \times Y+X^{\prime} & A 18 \\
\alpha & (59) & X^{\prime} \times Y^{\prime}=X \times Y+Y+X^{\prime}=X \times & (58),(57), M+, T R=, C A+, \\
& & Y+X+Y^{\prime}=X \times Y^{\prime}+Y^{\prime} & S U=, A 16, A 18, M+
\end{array}
$$

from which (55) follows by $R M I,(56),(57),(59)$.

Now comes $C \times$.

$$
\begin{array}{llll}
\top & (60) & 0 \times Y=Y \times 0 & 0 \times, \times 0, T R= \\
\alpha & (61) & X \times Y=Y \times X & \text { НуротнESIS } \\
\alpha & (62) & X^{\prime} \times Y=X \times Y+Y=Y \times X+Y= & (55),(61), M+, A 18, T R= \\
& Y \times X^{\prime} & \\
\top \quad(63) & X \times Y=Y \times X & (60),(61),(62), R M I
\end{array}
$$

$\times$ distributes over + by $D \times+$.

$$
\begin{array}{lclll}
\top & (64) & (Y+Z) \times 0=Y \times 0+Z \times 0 & \times 0,+0, M+ \\
\alpha & (65) & (Y+Z) \times X=Y \times X+Z \times X & \text { Hypothesis } \\
\top & (66) & (Y+Z) \times X^{\prime}=(Y+Z) \times X+(Y+Z) & A 18 \\
\top \quad & (67) & Y \times X^{\prime}+Z \times X^{\prime}=Y \times X+Y+ & A 18, M+, N+ \\
& & (Z \times X+Z) & \\
\alpha & (68) & (Y+Z) \times X^{\prime}=Y \times X+Z \times X+ & (65),(66), M+, T R= \\
& (Y+Z) & \\
\alpha & (69) & (Y+Z) \times X^{\prime}=Y \times X^{\prime}+Z \times X^{\prime} & (68), A+, C A+, M+,(67), \\
& & & T R= \\
\top & (70) & (Y+Z) \times X=Y \times X+Z \times X & (64),(65),(69), R M I
\end{array}
$$

The form of $D \times+$ given above then follows from (70), $C \times, T R=$. Similarly, the right monotony law $M \times$ yields the left monotony law $N \times$, exactly as in the addition case, once the commutative principle $C \times$ has been established. Here is $A \times$. 


$\begin{array}{llll}\top & (71) & X \times Y \times 0=X \times(Y \times 0) & \times 0, N \times, \times 0, T R= \\ \alpha & (72) & X \times Y \times Z=X \times(Y \times Z) & \text { HYPOTHESIS } \\ \top & (73) & X \times Y \times Z^{\prime}=X \times Y \times Z+X \times Y & A 18 \\ \top & (74) & X \times\left(Y \times Z^{\prime}\right)=X \times(Y \times Z)+X \times Y & A 18, N \times, D \times+ \\ \alpha & (75) & X \times Y \times Z^{\prime}=X \times\left(Y \times Z^{\prime}\right) & (72), N+,(73),(74) \\ \top & (76) & X \times Y \times Z=X \times(Y \times Z) & (71),(72),(75), R M I\end{array}$

$C A \times$ is again immediate from $C \times, A \times$, ending our survey of the most elementary arithmetical laws. Let us pause for a bit of stock-taking.

We introduced in II the notions of numerical equation and quasi-numerical equation, comprising respective classes NEQ and NEQQ. The slightly wider class NEQQ, in particular, is the class of all the ordinary statements of intuitive arithmetic: $2+2=4,5 \times 3=17$, and the like. Without troubling ourselves further on the matter, we take it as understood which of these statements are to be taken as true. And we lay down immediately

Theorem 1. For every true quasi-numerical equation $A, A$ is a theorem of $\mathrm{R}^{\sharp}$

To prove Theorem 1, it suffices to observe that every quasi-number $J$ may be, by possibly repeated application of the recursive axioms for + and $\times$, perhaps also using $S U=, T R=$, transformed into a number $J^{\star}$, such that $J=J^{\star}$ is a theorem of $\mathrm{R}^{\sharp}$. On severely finitistic argument (e.g., that indulged in by one's third grade teacher), only one such $J^{\star}$ can be churned out by this procedure. So, given a quasi-numerical equation, it is correct, in the sense of II, iff, on application of this procedure, $I^{\star}$ and $J^{\star}$ are the same number, if $I=J$ was the given equation. So, if $I=J$ is correct, all of $I=I^{\star}, J=J^{\star}$, $I^{\star}=J^{\star}$ are theorems of $\mathrm{R}^{\sharp}$, the last by $R=$. Accordingly, $I=J$, if correct, is a theorem also, by $T R=$.

With only a little more effort, we can form the general notion of a correct arithmetical equation. For suppose that we are given any term $T$. Without loss of generality, we may take $T$ to have been built up from variables and numbers by + and $\times$. (For, given +1 , we may now trade in any term $U^{\prime}$ for $U+1$, while we may by definition trade in the successors of 0 for numbers.) By the distributive, associative, and commutative laws from + and $\times$, we may go on to transform $T$ into $T^{\star}$, where $T^{\star}$ is of the form $T_{1}+\ldots+T_{N}$, and where each $T_{I}$ is of the form $J \times X_{1} \times \ldots \times X_{M}$, where $M$ is a number and the $X_{K}$ are variables. (The same variable, of course, can occur repeatedly in such a 
$T_{I}$.) On settling degenerate cases imposing some further criteria of standard form, it is clear that for every term $T$ of $\mathscr{L}^{\sharp}$ there is only one $T^{\star}$ in standard form to which $T$ corresponds, and that, for this $T^{\star}$, we can prove $T=T^{\star}$ in $\mathrm{R}^{\sharp}$. Similarly, for arbitrary terms $T, U, T=U$ is a correct arithmetical equation just in case $T^{\star}$ and $U^{\star}$ are the same (standard polynomial) term. So,

Theorem 2. For every correct arithmetical equation $A, A$ is a theorem of $R^{\sharp}$.

Since we explained how to prove Theorem 2 in the process of stating it, and since the reader need only advance from 3rd grade arithmetic to 8th grade algebra to convince himself which are the correct equations, we need dwell no further this theorem.

It is presumably as clear which are the false quasi-numerical equations as which are the true ones. And the requisite theorem is forthcoming just as easily.

Theorem 3. For every false quasi-numerical equation $A, \sim A$ is a theorem of $R^{\sharp}$.

For proof of Theorem 3, we let an example suffice by showing $2+2 \neq 7$.

$\begin{array}{llll}\alpha & (77) & 2+2=7 & \text { Hypothesis } \\ \alpha & (78) & 4+0=4+2^{\prime} & (77), \text { ThEOREM } 1 \\ \alpha & (79) & 0=2^{\prime} & (78), C+, M- \\ \top & (80) & 0 \neq 2^{\prime} & A 12 \\ \alpha & (81) & \perp & (79),(80), \perp I \\ \top & (82) & 2+2 \neq 7 & (77),(81), \sim I, D \neq\end{array}$

Similarly, given Theorem 1 , and the subtraction principle $M-$, it is evident that every false quasi-numerical equation $A$ will imply $0=K^{\prime}$ for some number $K$, whence, by $A 12$ and modus tollens, we prove $\sim A$ in $\mathrm{R}^{\sharp}$.

Theorem 3 is one other side of the Theorem 1 coin. We can prove the true equations, and prove the negations of the false ones, when they are quasinumerical. Moreover, these proofs work relevantly in just about the same way that they work classically; there are no surprises, nor were any likely to have been expected. (In fact, save for our purpose in exhibiting the relevant system and actually accustoming the reader to it, we might have employed

Australasian Journal of Logic (18:5) 2021, Article no. 4 
various shortcuts.)

However, there is a more salient side to the Theorem 1 coin. For, besides demonstrating $\sim A$ whenever $A$ is a false equation, we should like some believable assurances that $A$ itself is undemonstrable. Having demonstrated $2+2 \neq 7$, it profits us little if we can also demonstrate $2+2=7$. And here exactly is where classical technique leaves us flat. Such assurance as it gives us is not believable, save on more faith than we had when we undertook to do formal arithmetic in the first place. A simple arithmetic faith we shall perhaps concede, so long as it does not become too platonistic. But a super arithmetic faith we do not concede. And we demonstrate below, for $R^{\sharp}$, that it is superfluous.

Next let us ask, "What kind of theorem stands to Theorem 2 as Theorem 3 stands to Theorem 1?" Strictly speaking, the answer is "None." For, e.g., although $x=0$ is an incorrect equation, the open formula $x \neq 0$ is likewise arithmetically invalid.

However, by passing to universal closures of arbitrary equations, we may state and prove the appropriate generalization of Theorem 2. Specifically, where $A$ is any formula, let $\forall A$ be any formula $\forall X_{1} \ldots \forall X_{N} A$, such that all the free variables of $A$ are among $X_{1}, \ldots, X_{N}$. (All such universal closures of $A$ being provably equivalent in $\mathrm{RQ}$ and a fortiori in $\mathrm{R}^{\sharp}$, we may let $\forall A$ serve indifferently for any of them.) Similarly, let $\exists A$ be any existential closure $\exists X_{1} \ldots \exists X_{N} A$ of $A$. Then

Theorem 4. For every incorrect arithmetical equation $A, \exists \sim A$ and hence $\sim \forall A$ are theorems of $\mathrm{R}^{\sharp}$.

Proof. We leave it to the reader to demonstrate that, where $A\left[X_{1}, \ldots, X_{N}\right]$ is an incorrect equation with free variables $X_{1}, \ldots X_{N}$, there are numbers $I_{1}, \ldots, I_{N}$ such that the quasi-numerical equation $A\left[I_{1}, \ldots, I_{N}\right]$ is arithmetically false. By Theorem $2, \sim A\left[I_{1}, \ldots, I_{N}\right]$ is a theorem of $\mathrm{R}^{\sharp}$, whence by existential generalization so is $\exists X \sim A\left[X_{1}, \ldots, X_{N}\right]$, whence so by quantifier interchange is $\sim \forall X A\left[X_{1}, \ldots, X_{N}\right]$, ending the proof of Theorem 4 .

Theorems 1-4 are trivial results at the bottom of the arithmetical hierarchy. Nevertheless, they are important here as indications of the stability of $R^{\sharp}$. On the most basic theorems of formalized arithmetic, $R^{\sharp}$ agrees with $C^{\sharp}$ and, we presume, with almost everybody's arithmetical intuitions. And, as in the case of the theorem which it generalizes, there is more than one side to Theorem 3. Again, we seek proof that every incorrect arithmetical equation 
is underivable. And the classical hang-up over consistency still applies to $C^{\sharp}$ on this point; we can only have the proof we seek, classically, if we can prove $C^{\sharp}$ consistent. And we cannot prove $C^{\sharp}$ consistent, classically, save on greater arithmetical faith than $C^{\sharp}$ itself formalizes. In $R^{\sharp}$, on the other hand, the consistency hang-up vanishes, so far as basic arithmetic is concerned, so that the desired counterpart of Theorem 3 will also follow in a wholly elementary way, below.

\section{IX}

In the last section, we devoted ourselves to proof of the most elementary propositions of anybody's formal arithmetic. In this section, we continue with this project. However, we shall begin to note an increasing divergence between $C^{\sharp}$ and $J^{\sharp}$ on the one hand and $R^{\sharp}$ on the other. For, while we shall find no divergences here, within the classical vocabulary itself, between $C^{\sharp}$ and $R^{\sharp}$, we shall find theorems which hold materially such that their natural relevant counterparts fail, for good relevant reasons. (However, the possibility of future formal evidence which undermines the stability of $R^{\sharp}$, or of $C^{\sharp}$ for that matter, must always be left open.)

First, let us return to a topic left over from the beginning of VI, with respect to which we are more classical, perhaps, than we should like to be. We stated in VI but did not prove two principles relation to substitution properties of equality. They were

$$
\begin{array}{ll}
T=. & Y=Z \rightarrow T[Y]=T[Z] \\
S=. & Y=Z \rightarrow . A[Y] \rightarrow A[Z]
\end{array}
$$

where $T[X], A[X]$ are respectively an arbitrary term and an arbitrary formula, in which $X$ may (but need not) occur free.

We prove $T=$ by induction on the length of $T[X]$. In the base case, $T[X]$ is a formal atom. We distinguish two subcases.

Subcase 1.1. $T[X]$ is $X$. Then $T=$ is in this case of the form $Y=Z \rightarrow Y=Z$, which is a theorem of $R^{\sharp}$ at the purely sentential level.

Subcase 1.2. $T[X]$ is a formal atom $U$ distinct from $X$. In this case the substitution is vacuous, and $T=$ is of the form $Y=Z \rightarrow U=U$. By $R=$, $=\leq T^{\star}$, this is a theorem of $R^{\sharp}$.

Australasian Journal of Logic (18:5) 2021, Article no. 4 
We now suppose on inductive hypothesis that $T=$ holds for arbitrary terms $U[X], V[X]$, and we wish to show that it continues to hold when $T[X]$ is of one of the forms $U[X]^{\prime}, U[X]+V[X], U[X] \times V[X]$, giving rise to one of the following 3 subcases.

Subcase 2.1. $T[X]$ is $U[X]^{\prime}$. On inductive hypothesis, we prove $Y=Z \rightarrow$ $U[Y]=U[Z]$. By $S U=$, Replacement, we have $Y=Z \rightarrow U[Y]^{\prime}=U[Z]^{\prime}$, which was to be proved in this case.

Subcase 2.2. $T[X]$ is $U[X]+V[X]$. Then

$$
\begin{array}{llll}
\alpha & (1) \quad Y=Z & \text { Hypothesis } \\
\alpha & (2) \quad U[Y]=U[Z] & \text { By Inductive Hypothesis, (1), } \\
& & \rightarrow E \\
\alpha & (3) \quad V[Y]=V[Z] & \text { By Inductive Hypothesis, }(1), \\
& & \rightarrow E \\
\alpha & (4) \quad U[Y]+V[Y]=U[Z]+V[Y] & (2), M+ \\
\alpha & (5) \quad U[Z]+V[Y]=U[Z]+V[Z] & (3), N+ \\
\alpha & (6) \quad U[Y]+V[Y]=U[Z]+V[Z] & (4),(5), T R= \\
\top & (7) \quad Y=Z \rightarrow T[Y]=T[Z] & (1),(6), \rightarrow I
\end{array}
$$

Subcase 2.3. $T[X]$ is $U[X] \times V[X]$. The argument is exactly the same as in subcase 2.2., using $M \times, N \times$ instead of $M+, N+$.

This completes the inductive argument, and establishes $T=$ without restriction in $\mathrm{R}^{\sharp}$. We now prove $S=$ similarly, by induction on the length of $A[X]$. In the base case, $A[X]$ is an atomic formula $T[X]=U[X]$. We wish to show the theoremhood of $Y=Z \rightarrow T[Y]=U[Y] \rightarrow T[Z]=U[Z]$. So

$\begin{array}{llll}\alpha & (8) & Y=Z & \text { Hypothesis } \\ \rho & (9) & T[Y]=U[Y] & \text { Hypothesis } \\ \alpha & (10) & T[Y]=T[Z] & (8), T= \\ \alpha & (11) & U[Y]=U[Z] & (8), T= \\ \alpha \rho & (12) & T[Z]=U[Z] & (9),(10),(11), T R= \\ \top & (13) & Y=Z \rightarrow . A[Y] \rightarrow A[Z] & (9),(12), \rightarrow I,(8), \rightarrow I\end{array}$

This disposes of the base case for $S=$, and we now assume on inductive hypothesis that $S=$ holds for arbitrary formulas $B[X], C[X]$. We wish to 
show that it continues to hold when $A[X]$ is one of the forms $\sim B[X], B[X] \wedge$ $C[X], B[X] \rightarrow C[X], \forall X B[X], \forall W B[X]$, giving rise to the following 5 subcases.

Subcase 3.1. $A[X]$ is $\sim B[X]$. On inductive hypothesis and an elementary appeal to contraposition, $S=$ holds in this case.

Subcase 3.2. $A[X]$ is $B[X] \wedge C[X]$. Then

$\begin{array}{llll}\alpha & (14) & Y=Z & \text { Hypothesis } \\ \alpha & (15) & B[Y] \rightarrow B[Z] & \text { By Inductive Hypothesis, (14) } \\ \alpha & (16) & C[Y] \rightarrow C[Z] & \text { By Inductive Hypothesis, (14) } \\ \rho & (17) & B[Y] \wedge C[Y] & \text { Hypothesis } \\ \alpha \rho & (18) & B[Z] \wedge C[Z] & (17), \wedge E,(15), \rightarrow E,(16), \rightarrow E, \wedge I \\ \top & (19) & Y=Z \rightarrow . A[Y] \rightarrow A[Z] & (17),(18), \rightarrow I,(14), \rightarrow I\end{array}$

Subcase 3.3. $A[X]$ is $B[X] \rightarrow C[X]$. Like 3.2 , left to reader.

Subcase 3.4. $A[X]$ is $\forall X B[X]$. In this case, the replacement in $A[Y], A[Z]$ is vacuous, and $S=$ is of the form $Y=Z \rightarrow A \rightarrow A$, which is a theorem of $\mathrm{R}^{\sharp}$ by $=\leq \top^{\star}$.

Subcase 3.5. $A[X]$ is $\forall W B[X]$, where $W$ is distinct from $X$. We suppose, to begin with, that $W$ is also distinct from $Y, Z$. Then

$\begin{array}{llll}\alpha & (20) & Y=Z & \text { Hypothesis } \\ \alpha & (21) & B[Y] \rightarrow B[Z] & \text { By Inductive Hypothesis, (20) } \\ \rho & (22) & \forall W B[Y] & \text { Hypothesis } \\ \alpha \rho & (23) & B[Z] & (22), \forall E,(21), \rightarrow E \\ \alpha \rho & (24) & \forall W B[Z] & (23), \forall I \\ \top & (25) & Y=Z \rightarrow . A[Y] \rightarrow A[Z] & (22),(24), \rightarrow I,(20), \rightarrow I\end{array}$

If $W$ is one of $Y, Z$ in subcase 3.5, the situation is complicated slightly by the steps which we took in II, on a rather informal level, to avoid confusion of bound variables. Note, at least, that the step of $\forall I$ that produces (24) will not go through if, say, $W$ is the same variable as $Y$, since in that case $W$ will be free in $\alpha$, and hence in $\alpha \rho$, contravening the restriction on our $\forall I$ rule.

However, the point just made doesn't really arise in our inductive arguAustralasian Journal of Logic (18:5) 2021, Article no. 4 
ment. For, on our suggested convention, if $A[X]$ is of the form, say, $\forall Y B[X]$, then $A[Y]$ will be formed in the following steps. First, replace $\forall Y B[X]$ with some alphabetic variant $\forall V B^{\star}[X]$ thereof, where $V$ is some variable fresh to the context and $B^{\star}[X]$ is the result of systematic replacement of free $Y$ with $V$. Second, let $A[Y]$ be $\forall V B^{\star}[Y]$. On a trivial strengthening of our inductive hypothesis, the above argument then goes through as before, since step (24) is no longer blocked if we generalize on fresh $V$, while we may rewrite bound variables at will by $R B V$ to replace $V$ once more with $W$ in (24), if appropriate. Similar remarks apply if the $W$ of the subcase is $Z$, ending the inductive argument and completing the proof of $S=$ for $\mathrm{R}^{\sharp}$.

Now that we have $T=$ and $S=$ in full generality, together with the various arithmetical laws developed in the last section, we can be somewhat more free and easy with respect to some aspects of our proof procedure. In particular, most of our special replacement laws (e.g., $S U=, M+, N+, M \times$, $N \times$, and even $=\leq \top$ ) now become instances of $T=$, and may be referred to as such. Similarly, matters that we had to be careful about above (e.g., not making illicit appeals to the commutativity or associativity of,$+ \times$, or the distribution of $\times$ over + ) have now lapsed, since terms that are equal under elementary arithmetic transformations have now been established to be intersubstitutable in any context. Since the reader may be taken to be familiar with these transformations, we need call attention to them henceforth only when some special point relative to the formalism of $R^{\sharp}$ is involved, otherwise leaving the transformations tacit.

While we are at it, we also adopt some more common arithmetical notation. In particular, just as we have reserved simple juxtaposition between formulas (and especially between tags) for the fusion connective $\circ$, so we shall now follow ordinary arithmetical convention and use simple juxtaposition between terms for multiplication. Henceforth we shall also use

$$
\begin{array}{ll}
D T^{0} . & T^{0}={ }_{\mathrm{DF}} 1 \\
D T^{1} . & T^{1}={ }_{\mathrm{DF}} T \\
D T^{2} . & T^{2}={ }_{\mathrm{DF}} T T,
\end{array}
$$

etc. And we shall also revert to the ordinary practice, usually, of using additive notation for the successor function. I.e.,

$$
D+1 . \quad T+1=_{\mathrm{DF}} T^{\prime}
$$

Australasian Journal of Logic (18:5) 2021, Article no. 4 
Strictly speaking, none of the "definitions" just entered are formal definitions at all. (For it should be recalled that our formal systems are abstract, and that they are thus quite independent of any notational habits which it might be useful for us to form, or which (as is more to the point here) most of us have already formed.) We have not, in particular, defined anything like an exponential function in $D T^{0}, D T^{1}$, etc. We have merely indicated how we are going to use certain pieces of notation. And, so far as $D+1$ is concerned, we are introducing a deliberate ambiguity into our notation. Henceforth, when the reader sees, say, the piece of notation " $0+1$," only contextual clues will tell him which of the distinct formal objects $0^{\prime}, 0+1$ is being named by that piece of notation. In view, however, of theorems proved above, the successor notation has fully served its purpose, save where very great compactness is desired, and it is time to replace it with a notation that most of us see through more quickly, without troubling ourselves over ambiguities that by now have become harmless.

There is, however, one respect in which continuing attention to the successor function is worthwhile. 0, as we all know, is a very special number, and it is sometimes convenient to restrict our attention to positive integers. E.g., where $A[X]$ is an open formula with single free variable $X$, a quite natural way to assert that this formula is satisfied by all positive integers is to assert $\forall X A\left[X^{\prime}\right]$. In order to make this use of the successor function psychologically immediate, we shall often assert $\forall X A\left[X^{+}\right]$instead, entering the trivial definition

$$
D^{+} . \quad T^{+}=_{\mathrm{DF}} T^{\prime}
$$

So much, presently, for convenient notation. We extend it and bend it as is useful.

Our next formal topic is the natural order on natural numbers. We have already defined $T \leq U$ as $\exists X(T+X=U)$ and $T<U$ as $\exists X(T+X+1=U)$. We enter the following familiar and related definitions.

$$
\begin{array}{ll}
D \geq . & T \geq U=_{\mathrm{DF}} U \leq T \\
D>. & T>U=_{\mathrm{DF}} U<T \\
D \nless . & T \nless U=_{\mathrm{DF}} \sim(T<U) \\
D \not \leq . & T \not \leq U=_{\mathrm{DF}} \sim(T \leq U) \\
D \ngtr . & T \nsupseteq U=_{\mathrm{DF}} \sim(T \geq U) \\
D \ngtr . & T \ngtr U=_{\mathrm{DF}} \sim(T>U)
\end{array}
$$

Australasian Journal of Logic (18:5) 2021, Article no. 4 
We may also write, without the courtesy of a formal definition, $T \leq U \leq V$, etc., for $T \leq U \wedge U \leq V$, etc.

The above definitions, together with those already entered above, are straightforward and familiar. However, relevant order is a subtle topic, and materially equivalent order notions (e.g., $T<U$ and $T \nsupseteq U$ ) are not necessarily relevantly equivalent, even though the proof of their material equivalence is quite straightforward in $\mathrm{R}^{\sharp}$. In particular, let us think about the diversity claim $T \neq U$. This says, relevantly, that if one assumes $T=U$, one deduces something objectionable - in particular, $\perp$.

But there is another diversity claim worth notice, which is prima facie stronger. Namely, we may be able to prove either that $T$ is bigger than $U$ or that $U$ is bigger than $T$. So, to make room for this stronger diversity claim, we define

$$
D \lessgtr \quad T \lessgtr U=_{\mathrm{DF}} T<U \vee U<T
$$

Note that our "strong" diversity $\lessgtr$, unlike $\neq$, does not involve negation at all, if we consider the existential quantifier entering into the definition of $<$ as a "positive" logical particle. And, while our theory of negation is rather classical, we wish to respect some intuitionist intuitions, e.g., those usually attributed to Griss, which see underlying mathematical insights as negationfree. We are not bound to these intuitions, but it is convenient from a deductive point of view, and philosophically salutary, to pursue them as far as they go. Indeed, exactly the kinds of philosophical motivation that cause us to forswear $\gamma$ as a primitive rule, much as we should perhaps like to find it admissible anyway for $\mathrm{R}^{\sharp}$, urge a concentration on "positive" reasoning. We are not so arithmetically faithless as to expunge $\sim$ from our arithmetical vocabulary, or to deny it its ordinary properties. But, given the unusual arguments (e.g., those of Russell and Gödel) that rest on strange quirks of these ordinary properties, we are cautious enough to appeal to negationfree reasoning whenever we can carry it through, and to argue to material implications (as facts about the natural numbers), not to argue through such implications (as if they were the arithmetical laws).

When thinking from a negation-free point of view, $0=1$, which corresponds to the technically absurd intuitionist $\mathrm{F}$, is about as objectionable a formula as one might find. $0=2,6=12$, and so forth are pretty bad too, though perhaps they are not quite so bad. However, noting that any such direct arithmetic falsehood will imply $\exists X\left(0=X^{+}\right)$, and that it was the nega- 
tion of this assertion, essentially, which was the only one of our arithmetic principles that we might take to involve negation in a central way (though it is certainly involved also in $R M I$ ), we might take a formula of the sort just cited as a positive falsehood likely to be implied by arbitrary positive falsehoods. Indeed, we might think of ourselves as having a hierarchy of positive falsehoods, beginning with our old F. So let us define

$$
\begin{array}{ll}
D F_{1} . & F_{1}=_{D F} 0=1 \\
D F_{2} . & F_{2}={ }_{D F} 0=2
\end{array}
$$

etc. And then the join of positive falsehoods contemplated above is just

$$
D \mathrm{~F}_{\infty} . \quad \mathrm{F}_{\infty}={ }_{\mathrm{DF}} \exists x\left(x^{+}=0\right)
$$

On this scheme, we might as well express our axiom $A 12$ simply as

$$
A 12^{\star} . \quad \sim \mathrm{F}_{\infty}
$$

And it is of course trivial, for $I=1,2,3, \ldots$, that

$$
\begin{array}{ll}
1 \leq I . & \mathrm{F}_{1} \rightarrow \mathrm{F}_{I} \\
I \leq \infty . & \mathrm{F}_{I} \rightarrow \mathrm{F}_{\infty}
\end{array}
$$

are theorems of $R^{\sharp}$. And, still on the subject of $F_{\infty}$, it is equally trivial that

$$
0<\infty . \quad \mathrm{F}_{\infty} \leftrightarrow 0<0
$$

is a theorem of $\mathrm{R}^{\sharp}$, by definitions and the theorem $0+X=X$.

Corresponding to each of these falsehoods we can define an appropriate "positive negation." I.e.,

$$
\begin{array}{ll}
D \sim_{1} . & \sim_{1} A={ }_{\mathrm{DF}} A \rightarrow \mathrm{F}_{1} \\
D \sim_{2} . & \sim_{2} A={ }_{\mathrm{DF}} A \rightarrow \mathrm{F}_{2}
\end{array}
$$

etc. Similarly,

$$
D \sim_{\infty} . \quad \sim_{\infty} A=_{\mathrm{DF}} A \rightarrow \mathrm{F}_{\infty}
$$

Ordinarily, we shall leave out the subscript on the final connective, writAustralasian Journal of Logic (18:5) 2021, Article no. 4 
ing simply $\sim A$ for $\sim_{\infty} A$. Similarly, we may have a corresponding string of intuitionist negations, on the plan

$$
\begin{array}{ll}
D \neg_{1} . & \neg_{1} A==_{\mathrm{DF}} A \supset \mathrm{F}_{1} \\
D \neg_{2} . & \neg_{2} A={ }_{\mathrm{DF}} A \supset \mathrm{F}_{2} \\
\vdots & \\
D \neg_{\infty} . & \neg_{\infty} A=_{\mathrm{DF}} A \supset \mathrm{F}_{\infty}
\end{array}
$$

In this case, it will be recalled that we have already reserved $\neg_{1} A$ for our intuitionist $\neg A$, in keeping with tradition but on another plan than that just set out for $\sim A$.

As a final interesting order related definition, we set

$$
D \cong . \quad T \cong U=_{\mathrm{DF}} T \leq U \wedge T \geq U
$$

Again, we should expect a material, but not necessarily a relevant equivalence, provable in $R^{\sharp}$, between $T=U$ and $T \cong U$.

Some expected order theorems are not very hard to prove, such as

$$
\begin{array}{ll}
\leq \rightarrow \ngtr . & T \leq U \rightarrow T \ngtr U \\
<\rightarrow \ngtr . & T<U \rightarrow T \ngtr U \\
>\rightarrow \not . & T>U \rightarrow T \not U \\
\geq \rightarrow \nless . & T \geq U \rightarrow T \nless U \\
=\rightarrow \leq . & T=U \rightarrow T \leq U \\
<\rightarrow \leq . & T<U \rightarrow T \leq U \\
R \leq . & T \leq T \\
\infty \rightarrow<. & \mathrm{F}_{\infty} \rightarrow X<Y \\
\leq<<. & T \leq U<V \rightarrow T<V \\
<\leq<. & T<U \leq V \rightarrow T<V \\
T R<. & T<U<V \rightarrow T<V \\
T R \leq . & T \leq U \leq V \rightarrow T \leq V \\
I R<. & T \nless T
\end{array}
$$

We construct a derivation which suffices for some of these principles, leaving the rest to the reader.

Australasian Journal of Logic (18:5) 2021, Article no. 4 


\begin{tabular}{|c|c|c|c|}
\hline$\alpha$ & $(26)$ & $Z+1=0$ & HYPOTHESIS \\
\hline$\alpha$ & $(27)$ & $Y+Z+1=Y$ & $(26), T=$ \\
\hline$\alpha$ & $(28)$ & $0<Y$ & $(27), \exists I, D<$ \\
\hline$\rho$ & $(29)$ & $X<Y$ & HYPOTHESIS \\
\hline$\rho^{\star}$ & $(30)$ & $X+W+1=Y$ & HYPOTHESIS \\
\hline$\alpha \rho^{\star}$ & $(31)$ & $X+1+(W+Z+1)=Y$ & $(30), M+,(26), T=$ \\
\hline$\alpha \rho^{\star}$ & $(32)$ & $X+1<Y$ & $(31), D<$ \\
\hline$\alpha \rho$ & $(33)$ & $X+1<Y$ & (32), $\exists E,(30),(29)$ \\
\hline$\alpha$ & $(34)$ & $X<Y$ & (28), (29), (33), RMI \\
\hline T & $(35)$ & $\mathrm{F}_{\infty} \rightarrow X<Y$ & $(26),(34), \rightarrow I, R \exists E$ \\
\hline$\iota$ & $(36)$ & $Y \leq Z$ & HYPOTHESIS \\
\hline$\iota^{\star}$ & $(37)$ & $Y+U=Z$ & HYPOTHESIS \\
\hline$\rho^{\star} \iota^{\star}$ & $(38)$ & $X+W+U+1=Z$ & $(30), M+,(37), T=$ \\
\hline$\rho \iota$ & $(39)$ & $X<Z$ & (38), $\exists I, D<, \exists E$ \\
\hline$\top$ & $(40)$ & $X<Y \leq Z \rightarrow X<Z$ & $(36),(39), \rightarrow I,(29), \rightarrow I$ \\
\hline$\rho^{\star}$ & $(41)$ & $\exists Z(X+Z=Y)$ & $(30), \exists I$ \\
\hline$\top$ & $(42)$ & $X<Y \rightarrow X \leq Y$ & $\begin{array}{l}(30), \quad(41), \quad \exists E,(29), \rightarrow I, D<, \\
D \leq\end{array}$ \\
\hline$\omega$ & $(43)$ & $X+Z+1=X$ & HYPOTHESIS \\
\hline$\omega$ & $(44)$ & $\mathrm{F}_{\infty}$ & (43), $M-, \exists I, D F_{\infty}$ \\
\hline$\top$ & $(45)$ & $\sim(X<X)$ & (43), (44), $\rightarrow I, \exists E, D<, D \sim$ \\
\hline T & $(46)$ & $\sim(X<X)$ & $(45), A 12^{\star}$ \\
\hline$\beta$ & $(47)$ & $Z<Y$ & HYPOTHESIS \\
\hline$\beta^{\star}$ & $(48)$ & $Z+W^{\prime}=Y$ & HYPOTHESIS \\
\hline$\beta^{\star} \iota^{\star}$ & $(49)$ & $Y+U+W+1=Y$ & $(37),(48), T=$ \\
\hline$\top$ & $(50)$ & $Y \leq Z \rightarrow Z \ngtr Y$ & $\begin{array}{l}(49), D<,(46),(48), \exists E,(37), \\
\exists E,(47), \sim I,(36), \rightarrow I\end{array}$ \\
\hline
\end{tabular}

Steps of the derivation correspond to theorems stated above as follows: (35), $\infty \rightarrow<;(40),<\leq<;(42), I R<;(50), \leq \rightarrow \ngtr$. Other assertions are either trivial or follow in like manner to the above.

An innovation in the above derivation, which is much more telescoped than usual, is that, where $\Gamma$ is of the form $\exists X A[X]$, when definitions (e.g., of $<)$ are eliminated, we have been using $\Gamma^{\star}$ to tag (on hypothesis) what might count as an existential instantiation of $\Gamma$. (Thus having attached $\rho$ to step

Australasian Journal of Logic (18:5) 2021, Article no. 4 
(29) we attached $\rho^{\star}$ on this plan to step (30).) Officially, this changes nothing; $\rho^{\star}$ just counts as another piece of Greek, not to be confused with $\rho$ (or any other distinct piece of Greek). Nor do we allow any inference from, say, step (29) to step (30). Psychologically, however, it is useful to pretend that step (30) is an existential instantiation of step (29), and to use the $\star$ notation to reinforce this suggestion. Officially, however, we are just organizing the work for the various forms of $\exists E$, which accordingly appears as justification in the course of various arguments just above. And since each step continues to state, in a direct and immediate fashion, a theorem of $\mathrm{R}^{\sharp}$, no complex and devious restrictions on our "existential instantiation" rule are required, for the simple reason that, when the notation is unpacked, there is no such rule, but simply very ordinary procedures for passing from theorems to theorems.

Note that we have established, almost by the way

$\sim \rightarrow \sim . \quad \sim A \rightarrow \sim A$

We next develop some monotonicity properties.

$$
\begin{array}{ll}
M \leq^{\prime} . & T \leq U \leftrightarrow T^{\prime} \leq U^{\prime} \\
M<^{\prime} . & T<U \leftrightarrow T^{\prime}<U^{\prime} \\
M \leq+. & T \leq U \leftrightarrow T+W \leq U+W \\
M<+. & T<U \leftrightarrow T+W<U+W \\
M \lessgtr+. & T \lessgtr U \leftrightarrow T+W \lessgtr U+W \\
M \cong+. & T \cong U \leftrightarrow T+W \cong U+W
\end{array}
$$

We prove $M<+$ as typical of these properties, leaving the others to the reader.

$\begin{array}{llll}\alpha & (51) & X<Y & \text { HYpothesis } \\ \alpha^{\star} & (52) & X+Z+1=Y & \text { HYPOTHESIS } \\ \alpha^{\star} & (53) & X+W+Z+1=Y+W & (52), T= \\ \alpha & (54) & X+W<Y+W & (53), D<,(52), \exists E,(51) \\ \rho & (55) & X+W<Y+W & \text { HYPOTHESIS } \\ \rho^{\star} & (56) & X+W+Z+1=Y+W & \text { HYPOTHESIS } \\ \rho^{\star} & (57) & X+Z+1=Y & (56), M- \\ \rho & (58) & X<Y & (57), \exists I, D<,(56), \exists E,(55)\end{array}$

Australasian Journal of Logic (18:5) 2021, Article no. 4 
(51), (54), $\rightarrow I$, (55), (58), $\rightarrow I$, $D \leftrightarrow$

Here are some further easy theorems.

$$
\begin{array}{ll}
<+1 . & X+1>X \\
0<+1 . & X+1>0 \\
\leq \leftrightarrow+1 . & X \leq Y \leftrightarrow X<Y+1 \\
<\leftrightarrow+1 . & X<Y \leftrightarrow X+1 \leq Y
\end{array}
$$

Our deduction continues.

$\begin{array}{llll}\top & (60) & 0+X+1=X+1 & 0+ \\ \top & (61) & X<X+1 & (60), \exists I, D< \\ \top & (62) & 0<X+1 & (60), \exists I, D< \\ \alpha & (63) & X \leq Y & \text { HYPOTHESIS } \\ \alpha & (64) & X+1 \leq Y+1 & (63), M \leq+ \\ \alpha & (65) & X<Y+1 & (61),(64),<\leq< \\ \rho & (66) & X<Y+1 & \text { HYPOTHESIS } \\ \rho^{\star} & (67) & X+W+1=Y+1 & \text { HYPOTHESIS } \\ \rho^{\star} & (68) & X+W=Y & (67), M- \\ \rho & (69) & X \leq Y & (68), \exists I, D \leq,(67), \exists E,(66) \\ \top & (70) & X \leq Y \leftrightarrow X<Y+1 & (63),(65), \rightarrow I,(66),(69), \rightarrow I, \\ & & & D \leftrightarrow \\ \top & (71) & X+1 \leq Y \leftrightarrow X+1<Y+1 & (70) \\ \top & (72) & X<Y \leftrightarrow X+1 \leq Y & (71), M<+, \text { REPLACEMENT }\end{array}$

More order properties follow.

$$
\begin{array}{ll}
+\geq . & X+Y \geq X \\
+>. & X+Y^{+}>X \\
=0 \vee<. & X=0 \vee 0<X \\
=1 \vee<. & X=0 \vee X=1 \vee 1<X \\
=2 \vee<. & X=0 \vee X=1 \vee X=2 \vee 2<X, \text { etc. } \\
\leq \leftrightarrow<=. & X \leq Y \leftrightarrow X<Y \vee X=Y
\end{array}
$$

Australasian Journal of Logic (18:5) 2021, Article no. 4 
Here are their proofs.

\begin{tabular}{|c|c|c|c|}
\hline T & $(73)$ & $X+Y=X+Y$ & $R=$ \\
\hline 丁 & $(74)$ & $X \leq X+Y$ & (1), $\exists I, D \leq$ \\
\hline 丁 & $(75)$ & $X<X+Y^{+}$ & $(2),<+1, \leq<<$ \\
\hline 丁 & $(76)$ & $0=0 \vee 0<0$ & $R=, \vee I$ \\
\hline$\alpha$ & $(77)$ & $\nabla(X=0 \vee 0<X)$ & HYPOTHESIS \\
\hline 丁 & $(78)$ & $X+1=0 \vee 0<X+1$ & $0<+1, \vee I$ \\
\hline T & $(79)$ & $X=0 \vee 0<X$ & (76), (77), (78), RMI \\
\hline$\rho$ & $(80)$ & $X \leq Y$ & HYPOTHESIS \\
\hline$\rho^{\star}$ & $(81)$ & $X+Z=Y$ & HYPOTHESIS \\
\hline T & $(82)$ & $Z=0 \vee 0<Z$ & $(79)$ \\
\hline$\iota_{1}$ & $(83)$ & $Z=0$ & HYPOTHESIS \\
\hline$\rho^{\star} \iota_{1}$ & $(84)$ & $X=Y$ & $(81),(83), T=,+0$ \\
\hline$\iota_{2}$ & $(85)$ & $0<Z$ & HYPOTHESIS \\
\hline$\iota_{2}$ & $(86)$ & $X<X+Z$ & $(85), M<+,+0, T=$ \\
\hline$\rho^{\star} \iota_{2}$ & $(87)$ & $X<Y$ & $(81),(86), T=$ \\
\hline$\rho^{\star}$ & $(88)$ & $X=Y \vee X<Y$ & $(82),(83),(84),(85),(87), \vee E$ \\
\hline$\top$ & $(89)$ & $X \leq Y \rightarrow X=Y \vee X<Y$ & $(81), \rightarrow I, \exists E, D \leq,(80)$ \\
\hline T & $(90)$ & $X \leq Y \leftrightarrow X=Y \vee X<Y$ & $\begin{array}{l}(89), \quad R \leq, \quad T=, \quad<\rightarrow \leq, \quad \vee E, \\
D \leftrightarrow\end{array}$ \\
\hline T & $(91)$ & $X=0 \vee 1 \leq X$ & $(79),<\leftrightarrow+1$ \\
\hline T & $(92)$ & $X=0 \vee X=1 \vee 1<X$ & (91), (90), REPLACEMENT \\
\hline T & $(93)$ & $\begin{array}{l}X=0 \vee X=1 \vee X=2 \vee 2<X \\
\text { etc. }\end{array}$ & (92), In Like Manner \\
\hline
\end{tabular}

We now state the important trichotomy principles.

$$
\begin{array}{ll}
\text { TC1. } & X<Y \vee X=Y \vee X>Y \\
\text { TC2. } & X \leq Y \vee X>Y \\
\text { TC3. } & X<Y \vee X \geq Y \\
\text { TC4. } & X=Y \vee X \lessgtr Y \\
\text { TC5. } & X \leq Y \vee Y \leq X
\end{array}
$$

It will suffice to prove $T C 3, T C 1$, the other principles following trivially

Australasian Journal of Logic (18:5) 2021, Article no. 4 
using definitions and in like manner.

\begin{tabular}{|c|c|c|c|}
\hline T & (94) & $0<Y \vee 0=Y$ & $=0 \vee<$ \\
\hline | & $(95)$ & $0<Y \vee Y \leq 0$ & $=\rightarrow \leq$, (94), RePlacement \\
\hline & (96) & $X<Y \vee Y \leq X$ & HYPOTHESIS \\
\hline$x_{1}$ & (97) & $X<Y$ & HYPOTHESIS \\
\hline$\alpha_{1}$ & (98) & $X+1 \leq Y$ & $(97),<\leftrightarrow+1$ \\
\hline & (99) & $X+1 \leq Y \vee X+1=Y$ & $(98), \leq \leftrightarrow<=$ \\
\hline$\alpha_{2}$ & (100) & $Y \leq X$ & HYPOTHESIS \\
\hline$\alpha_{2}$ & (101) & $Y<X+1$ & $(100), \leq \leftrightarrow<=$ \\
\hline & (102) & $\begin{array}{l}X+1<Y \quad \vee \quad X+1=Y \quad \vee \\
Y<X+1\end{array}$ & $\begin{array}{l}(96),(97),(99),(100),(101) \\
\vee E\end{array}$ \\
\hline & (103) & $X<Y \vee Y \leq X$ & $(95),(96),(102), R M I$ \\
\hline & $(104)$ & $X<Y \vee X=Y \vee X>Y$ & $\begin{array}{l}(103), \leq \leftrightarrow<=, \quad \text { REPLACE- } \\
\text { MENT }\end{array}$ \\
\hline
\end{tabular}

So far, we have considered pure order properties, and we have related these properties to addition. We need also to relate them to multiplication, proving the following theorems.

$$
\begin{array}{ll}
+\geq X . & X Y^{+} \geq X \\
++>X . & X^{+} Y^{+}>X \\
\exists+>X . & \exists Z\left(Z Y^{+}>X\right) \\
M<\times . & X<Y \rightarrow X Z^{+}<Y Z^{+} \\
M \leq \times . & X \leq Y \rightarrow X Z \leq Y Z \\
C M<\times . & X Z<Y Z \rightarrow X<Y \\
C M \leq \times . & X Z^{+} \leq Y Z^{+} \rightarrow X \leq Y \\
Q M<\times . & X<Y \leftrightarrow X Z^{+}<Y Z^{+} \\
Q M \leq \times . & X \leq Y \leftrightarrow X Z^{+} \leq Y Z^{+} \\
Q M \cong \times . & X \cong Y \leftrightarrow X Z^{+} \cong Y Z^{+} \\
Q M \lessgtr \times . & X \lessgtr Y \leftrightarrow X Z^{+} \lessgtr Y Z^{+}
\end{array}
$$

Proofs are indicated as follows.

$$
\begin{array}{llll}
\top & (105) & X Y^{+}=X(Y+1)=X Y+X \geq X & D \geq \\
\top & (106) & X^{+} Y^{+}=X Y+X+Y+1>X & D>
\end{array}
$$

Australasian Journal of Logic (18:5) 2021, Article no. 4 


$\begin{array}{llll}\alpha & (107) & X<Y & \text { HYPOTHESIS } \\ \alpha & (108) & X 0^{+}<Y 0^{+} & (107), \times 1 \\ \rho & (109) & X Z^{+}<Y Z^{+} & \text {HYPOTHESIS } \\ \rho & (110) & X Z^{+}+X<Y Z^{+}+X & (109), M<+ \\ \alpha & (111) & X Z^{+}+X<Y Z^{+}+X & (107), M<+ \\ \alpha \rho & (112) & X Z^{++}<Y Z^{++} & (110),(111), T R< \\ \alpha & (113) & X Z^{+}<Y Z^{+} & (108),(109),(112), R M I \\ \top & (114) & X<Y \rightarrow X Z^{+}<Y Z^{+} & (107),(113), \rightarrow I \\ \top & (115) & X \leq Y \rightarrow X Z \leq Y Z & (114), \text { IN LIKE MANNER } \\ \iota & (116) & X Z<Y Z & \text { HYPOTHESIS } \\ \omega & (117) & Y \leq X & \text { HYPOTHESIS } \\ \omega & (118) & Y Z \leq X Z & (117),(115), \rightarrow E \\ \iota \omega & (119) & X Z<X Z & (116),(118),<\leq< \\ \iota \omega & (120) & \mathrm{F}_{\infty} & (119), 0+, M<+, 0<\infty \\ \iota \omega & (121) & X<Y & (120), \infty \rightarrow< \\ \iota & (122) & X<Y \vee Y \leq X & T C 3,(116),=\leq \top \\ \iota & (123) & X<Y & (122),(117),(121), \vee E \\ \top & (124) & X Z<Y Z \rightarrow X<Y & (116),(123), \rightarrow I \\ \top & (125) & X Z^{+} \leq Y Z^{+} \rightarrow X \leq Y & (124), \text { In LIKE MANNER } \\ & & & \end{array}$

The reader will have noted that (115) is for unrestricted $Z$, while its converse (125) is restricted to positive $Z$. Oppositely, (114) is for positive $Z$, while (124) is unrestricted. The other principles follow easily from definitions and those just set out, and their proofs are again left to the reader.

We have been seeking, so far as possible, to prove relevantly the theorems set out in $\S 38, \S 39$ of [IMM]. There are some differences in style which now need to be attended to. First, where we have proved statements of relevant implication, of course the corresponding material implications, being weaker, hold also in $R^{\sharp}$. The same comments hold where we have proved relevant equivalences.

Second, Kleene's technique for restricting theorems to positive integers differs from ours. Where, e.g., we proved

(i). $X Y^{+} \geq X$

the corresponding $[\mathrm{IMM}]$ statement, in our notation, is

Australasian Journal of Logic (18:5) 2021, Article no. 4 
(ii). $0 \neq Y \supset X Y \geq X$

and similarly in other cases. Since we wish to prove as many theorems as possible in the $C^{\sharp}$ vocabulary, we need to show that from theorems like (i) we can prove in $\mathrm{R}^{\sharp}$ theorems like (ii).

First, let us define a predicate in $\mathrm{R}^{\sharp}$ which says that $T$ is a positive integer. $0 \neq T$ is not a good choice. But $0<T$ works fine. So

$$
\text { DP. } \quad P T={ }_{\mathrm{DF}} 0<T
$$

Next we wish to show that we could have achieved the same result by relativizing to $P$ that we got by superscripting + . For this purpose, we shall prove in $\mathrm{R}^{\sharp}$

$$
Q P+. \quad \forall X(P X \rightarrow A[X]) \leftrightarrow \forall X A\left[X^{+}\right]
$$

It suffices to deduce each side of $Q P+$ from the other.

$\begin{array}{llll}\alpha & (126) & \forall X(P X \rightarrow A[X]) & \text { HYPothesis } \\ \alpha & (127) & P X^{+} \rightarrow A\left[X^{+}\right] & (126), \forall E \\ \top & (128) & P X^{+} & 0<+1, D P \\ \alpha & (129) & \forall X A\left[X^{+}\right] & (127),(128), \rightarrow E, \forall I \\ \rho & (130) & \forall X A\left[X^{+}\right] & \text {HYPOTHESIS } \\ \iota & (131) & P X & \text { HYPOTHESIS } \\ \iota^{\star} & (132) & X=W^{+} & \text {HYPOTHESIS } \\ \rho & (133) & A\left[W^{+}\right] & (130), \forall E \\ \rho \iota^{\star} & (134) & A[X] & (132),(133), T= \\ \rho \iota & (135) & A[X] & (132),(134), \exists E, D P, \\ \rho & (136) & \forall X(P X \rightarrow A[X]) & (131),(135), \rightarrow I, \forall I\end{array}$

In our illustrative case, this means that we can prove from (i)

(iii) $\quad P Y \rightarrow X Y \geq X$

Moreover, we have by $=0 \vee<$,

(iv) $Y=0 \vee P Y$

Australasian Journal of Logic (18:5) 2021, Article no. 4 
as a theorem of $R^{\sharp}$. By the directed replacement theorem in $R^{\sharp}$, we have from (iii) and (iv)

$$
\text { (v) } Y=0 \vee X Y \geq X
$$

after which (ii) is trivial in $R^{\sharp}$ employing $D$. (But note in this case that it is the material implication which is proved, as was to be expected.) We can employ this technique in general to get theorems like (ii) in $R^{\sharp}$ from theorems like (i) or (iii), but it is perhaps better to say that (i) and (iii) themselves are the relevant versions of the somewhat weaker assertion (ii).

We next turn to some theorems of $\S 39$ of [IMM] that we have hitherto skipped. They are

$$
\begin{array}{cccc}
\star 128 . & X+Y=0 \supset X=0 \wedge Y=0 & \star 129 . & X Y=0 \supset X=0 \vee Y=0 \\
\star 130 . & X+Y=1 \supset X=1 \vee Y=1 & \star 131 . & X Y=1 \supset X=1 \wedge Y=1 \\
& \star 133 . Z \neq 0 \supset . X Z=Y Z \supset X=Y
\end{array}
$$

An interesting feature of these theorems is that none of them hold in $\mathrm{R}^{\sharp}$ when $\supset$ is replaced everywhere by $\rightarrow$ (even if we change $Z \neq 0$ to our preferred $P Z$ in $\star 133$.). So we have some theorems here that, from the $\mathrm{R}^{\sharp}$ point of view, are seriously material. Let us look first for relevant counterparts, from which we can systematically prove them in $R^{\sharp}$.

$$
\begin{array}{ll}
<\rightarrow \lessgtr . & T<U \rightarrow T \lessgtr U \\
>\rightarrow \lessgtr . & T>U \rightarrow T \lessgtr U \\
\lessgtr \rightarrow \neq . & T \lessgtr U \rightarrow T \neq U \\
P \neq 0 . & P X \rightarrow X \neq 0 \\
P \rightarrow \vee . & (P X \rightarrow X) \supset X=0 \vee A \\
P \rightarrow \supset . & (P X \rightarrow A) \supset . X \neq 0 \supset A \\
R 128 . & P X \vee P Y \rightarrow P(X+Y) \\
R 129 . & P X \circ P Y \rightarrow P(X Y) \\
R 130 . & P X \circ 1<Y \rightarrow 1<X+Y \\
R 131 . & 1<X \circ 1<Y \rightarrow 1<X Y \\
R 133 . & X \lessgtr Y \rightarrow X Z^{+} \lessgtr Y Z^{+} \\
\lessgtr \rightarrow \vee . & (X \lessgtr Y \rightarrow A) \supset X=Y \vee A \\
\lessgtr \rightarrow \supset . & (X \lessgtr Y \rightarrow A) \supset . X \neq Y \supset A \\
<\rightarrow \neq . & T<U \rightarrow T \neq U
\end{array}
$$

Australasian Journal of Logic (18:5) 2021, Article no. 4 
Apart from $R 128-R 131$, most of the theorems in the above list are rather trivial from definitions and what has been already proved, being introduced here rather to simplify proofs. We prove $\lessgtr \rightarrow \neq, R 126-R 133, \lessgtr \rightarrow \vee$, leaving the others to the reader.

$\begin{array}{llll}\alpha & (137) & T \lessgtr U & \text { HYротHESIS } \\ \rho & (138) & T=U & \text { HYротHESIS } \\ \alpha \rho & (139) & T<T & (137),(138), T=, D \lessgtr, I D \vee \\ \alpha & (140) & T \neq U & (138),(139), I R<, \sim I \\ \top & (141) & T \lessgtr U \rightarrow T \neq U & (137),(140), \rightarrow I \\ \iota & (142) & P X & \text { HYPOTHESIS } \\ \iota & (143) & 0 \neq X & (142), D P,<\rightarrow \neq \\ \top & (144) & P X \rightarrow 0 \neq X & (142),(143), \rightarrow I \\ \omega & (145) & \nabla(X \lessgtr Y \rightarrow A) & \text { HYPOTHESIS } \\ \omega & (146) & X \lessgtr Y \vee X=Y & T C 4, D \nabla,=\leq \top \\ \omega & (147) & X=Y \vee A & (145),(146), \nabla E, \vee E \\ \top & (148) & (X \lessgtr Y \rightarrow A) \supset X=Y \vee A & (145),(147), \rightarrow I\end{array}$

Here are $R 128-R 133$.

$\begin{array}{llll}\alpha & (149) & 0<X & \text { Hypothesis } \\ \top & (150) & X \leq X+Y & +\geq \\ \alpha & (151) & 0<X+Y & (149),(150),<\leq< \\ \top & (152) & P X \rightarrow P(X+Y) & (149),(151), \rightarrow I, D P \\ \top & (153) & P Y \rightarrow P(X+Y) & (152), \text { In LiKe MANNER } \\ \top & (154) & P X \vee P Y \rightarrow P(X+Y) & (152),(153), \vee E \\ \rho & (155) & 0<Z & \text { HyPOTHESIS } \\ \rho^{\star} & (156) & Y=Z^{+} & \text {HypOTHESIS } \\ \top & (157) & X \leq X Z^{+} & +\geq X \\ \alpha \rho^{\star} & (158) & 0<X Y & (149),(157),<\leq<,(156), T= \\ \top & (159) & P X \circ P Y \rightarrow P(X Y) & (156),(158), \rightarrow I, \exists E \\ & & & (155),(149), \rightarrow I, D P\end{array}$

To prove $R 130$ use $M<+$; to prove $R 131$ use $M<\times$; to prove $R 133$ use $Q M \lessgtr \times$. Now let us prove the Kleene theorems $\star 128, \star 129, \star 133$, leaving

Australasian Journal of Logic (18:5) 2021, Article no. 4 
$\star 130, \star 131$ to the reader. (The latter are slightly case-ridden, but $R 130$, $R 131$ may be taken as their key ingredients.)

\begin{tabular}{|c|c|c|c|}
\hline T & (160) & $X=0 \vee P(X+Y)$ & (152), $P \rightarrow \vee, \supset E$ \\
\hline$T$ & (161) & $Y=0 \vee P(X+Y)$ & (160), In Like Manner \\
\hline | & (162) & $X=0 \wedge Y=0 \vee P(X+Y)$ & $(160),(161), \wedge I, D \wedge \vee$ \\
\hline T & (163) & $X+Y \neq 0 \vee X=0 \wedge Y=0$ & $\begin{array}{l}(162), C \vee, P \neq 0, \text { RePlace- } \\
\text { MENT }\end{array}$ \\
\hline | & (164) & $X+Y=0 \supset X=0 \wedge Y=0$ & (163), \\
\hline ] & (165) & $X=0 \vee \nabla(P Y \rightarrow P(X Y))$ & $\begin{array}{l}R 129, \rightarrow I, \quad P \rightarrow \vee, \quad R=, \quad \wedge I, \\
D \wedge \vee, D \nabla\end{array}$ \\
\hline | & (166) & $X=0 \vee Y=0 \vee P(X Y)$ & $\begin{array}{l}(165), P \rightarrow \vee, D \supset, \text { RePlace- } \\
\text { MENT }\end{array}$ \\
\hline$T$ & (167) & $X Y=0 \supset X=0 \vee Y=0$ & $\begin{array}{l}(166), P \neq 0, \text { RePlacement, } \\
C \vee, D \supset\end{array}$ \\
\hline$T$ & (168) & $X \lessgtr Y \rightarrow X Z^{+} \lessgtr Y Z^{+}$ & $R 133$ \\
\hline & (169) & $X=Y \vee X Z^{+} \lessgtr Y Z^{+}$ & $(168), \lessgtr \rightarrow \vee$ \\
\hline T & (170) & $X Z^{+}=Y Z^{+} \supset X=Y$ & $\begin{array}{l}(169), \lessgtr \rightarrow \neq, \text { REPLACEMENT, } \\
D \supset\end{array}$ \\
\hline & (171) & $P Z \rightarrow . X Z=Y Z \supset X=Y$ & $(170), \forall I, Q P+, \forall E$ \\
\hline & (172) & $Z \neq 0 \supset . X Z=Y Z \supset X=Y$ & $(171), P \rightarrow \supset, \supset E$ \\
\hline
\end{tabular}

We next prove the existence of quotients and remainders.

$\begin{array}{llll}\top & (173) & 0=X^{+} 0+0 \wedge 0<X^{+} & R=, 0<+, \wedge I \\ \top & (174) & \exists Q \exists R\left(0=X^{+} Q+R \wedge R<X^{+}\right) & (173), \exists I \\ \alpha & (175) & \exists Q \exists R\left(Y=X^{+} Q+R \wedge R<X^{+}\right) & \text {HYPOTHESIS } \\ \alpha^{\star} & (176) & Y=X^{+} Q+R \wedge R<X^{+} & \text {HYPOTHESIS } \\ \alpha^{\star} & (177) & Y+1=X^{+} Q+R+1 & (176), \wedge E, T= \\ \alpha^{\star} & (178) & R+1 \leq X^{+} & (176), \quad<E, \quad<+1 \quad(178), \leq \rightarrow<= \\ & & & \text { HYPOTHESIS } \\ \alpha^{\star} & (179) & R+1<X^{+} \vee R+1=X^{+} & (177),(180), \wedge I, \\ \alpha_{1} & (180) & R+1<X^{+} \\ \alpha^{\star} \wedge \alpha_{1} & (181) & \exists Q \exists R\left(Y+1=X^{+} Q+R \wedge R<X^{+}\right) & \exists I \\ & & & \text { HyPOTHESIS }\end{array}$

Australasian Journal of Logic (18:5) 2021, Article no. 4 


$$
\begin{aligned}
& \alpha^{\star} \wedge \alpha_{2} \quad(183) \quad Y+1=X^{+} Q+X^{+}=X^{+}(Q+1)+0 \quad \text { (177), (182), } T= \\
& \alpha^{\star} \wedge \alpha_{2} \quad \text { (184) } \exists Q \exists R\left(Y+1=X^{+} Q+R \wedge R<X^{+}\right) \quad \text { (183), } 0<+, \wedge I \text {, } \\
& \alpha^{\star} \wedge \alpha^{\star} \quad(185) \quad \exists Q \exists R\left(Y+1=X^{+} Q+R \wedge R<X^{+}\right) \quad \text { (179), } \\
& \text { (181), (182), } \\
& \top \quad \text { (186) } \exists Q \exists R\left(Y=X^{+} Q+R \wedge R<X^{+}\right) \quad \text { (185), (176), } \\
& \exists E,(173),(175) \text {, } \\
& R M I \\
& \begin{array}{llll}
\top & (187) & P X \rightarrow \exists Q \exists R(Y=X Q+R \wedge R<X) & (186), Q P+ \\
\top & (188) & X \neq 0 \rightarrow \exists Q \exists R(Y=X Q+R \wedge R<X) & (187), P \rightarrow \supset
\end{array}
\end{aligned}
$$

We may take any of (186), (187), (188) to be our desired principle $\exists Q \exists R$ asserting that division by a positive integer is always possible in $R^{\sharp}$, yielding a quotient and remainder. A principle $U Q U R$, asserting that the result of such division always yields a unique quotient and remainder, is considerably more bother. Again, $U Q U R$ is perhaps best considered a material principle, with various relevant counterparts. And it is most convenient to approach these counterparts via our weak equality relation $\cong$, whence a few more auxiliary theorems are in order.

$$
\begin{array}{ll}
Q<\infty . & X<X \leftrightarrow \mathrm{F}_{\infty} \\
\cong \infty=. & X \cong Y \leftrightarrow \mathrm{F}_{\infty} \vee X=Y \\
\cong \rightarrow \triangle . & X \cong Y \rightarrow \triangle(X=Y) \\
\cong \rightarrow \supset . & (A \rightarrow X \cong Y) \supset . A \supset X=Y
\end{array}
$$

Proofs of these are as follows.

$$
\begin{array}{llll}
\top & (189) & X<X \leftrightarrow \mathrm{F}_{\infty} & (45),(35), \wedge I, D \leftrightarrow \\
\top & (190) & \mathrm{F}_{\infty} \vee X=Y \rightarrow X \cong Y & (35),=\rightarrow \cong, \vee E \\
\alpha & (191) & X \leq Y \wedge Y \leq X & \text { HyPOTHESIS } \\
\alpha & (192) & X \leq Y \wedge(Y<X \vee Y=X) & (191), \leq \leftrightarrow<=, \quad \text { REPLACE- } \\
& & & \text { MENT } \\
\alpha & (193) & X \leq Y \wedge Y<X \vee Y=X & (192), D \wedge \vee, \text { REPLACEMENT } \\
\alpha & (194) & X<X \vee Y=X & (193), \leq<<, \text { REPLACEMENT } \\
\alpha & (195) & \mathrm{F}_{\infty} \vee X=Y & (194),(189), \text { REPLACEMENT }
\end{array}
$$

Australasian Journal of Logic (18:5) 2021, Article no. 4 


$\begin{array}{llll}\top & (196) & X \cong Y \leftrightarrow \mathrm{F}_{\infty} \vee X=Y & (191), D \cong,(195), \rightarrow I,(190) \\ \top & (197) & X \cong Y \rightarrow \perp \vee X=Y & (196), D \leftrightarrow, \wedge E, A 12^{\star}, \mathrm{RE}- \\ & & & \text { PLACEMENT } \\ \top & (198) & X \cong Y \rightarrow \triangle(X=Y) & (197), D \triangle \\ \rho & (199) & \nabla(A \rightarrow X \cong Y) & \text { HYPOTHESIS } \\ \iota & (200) & A \wedge X \neq Y & \text { HYPOTHESIS } \\ \rho \iota & (201) & X \cong Y & (199), \nabla E,(200), \wedge E, \rightarrow E \\ \rho \iota & (202) & \perp \vee X=Y & (197),(201), \rightarrow E \\ \rho \iota & (203) & X \neq Y & (200), \wedge E,(199),=\leq \top \\ \rho \iota & (204) & \perp \vee X=Y \wedge X \neq Y & (202),(203), \wedge I, D \wedge \vee \\ \rho \iota & (205) & \perp & (204), \perp I, \quad \text { REPLACEMENT } \\ & & & I D \vee \\ \rho & (206) & A \supset X=Y & (200),(205), \sim I, D \supset \\ \top & (207) & (A \rightarrow X \cong Y) \supset . A \supset X=Y & (199),(206), \rightarrow I, D \supset\end{array}$

The following is a key lemma for $U Q U R$.

$$
\text { RUQU. } \quad X^{+} Q_{1}+R_{1} \leq X^{+} Q_{2}+R_{2} \wedge R_{2}<X^{+} \rightarrow Q_{1} \leq Q_{2}
$$

We prove it as follows.

$\begin{array}{llll}\alpha & (208) & X^{+} Q_{1}+R_{1} \leq X^{+} Q_{2}+R_{2} \wedge & \text { НyротнеSis } \\ & & R_{2}<X^{+} & \\ \rho & (209) & Q_{2}<Q_{1} & \text { HyротнеSIS } \\ \rho & (210) & Q_{2}+1 \leq Q_{1} & (209),<\leftrightarrow+1 \\ \rho & (211) & X^{+} Q_{2}+X^{+} \leq X^{+} Q_{1} & (210), M \leq \times \\ \alpha & (212) & R_{2}<X^{+} & (208), \wedge E \\ \alpha & (213) & X^{+} Q_{2}+R_{2}<X^{+} Q_{2}+X^{+} & (212), M<+ \\ \alpha \rho & (214) & X^{+} Q_{2}+R_{2}<X^{+} Q_{1} & (213),(211),<\leq< \\ \top & (215) & X^{+} Q_{1} \leq X^{+} Q_{1}+R_{1} & +\geq \\ \alpha \rho & (216) & X^{+} Q_{2}+R_{2} \leq X^{+} Q_{1}+R_{1} & (214),(215),<\leq< \\ \alpha \rho & (217) & \mathrm{F}_{\infty} & (208), \wedge E,(216), \leq<<,(189) \\ \alpha \rho & (218) & Q_{1}<Q_{2} & (217),(35) \\ \alpha & (219) & Q_{2}<Q_{1} \rightarrow Q_{1} \leq Q_{2} & (218),<\rightarrow \leq,(209), \rightarrow I \\ \alpha & (220) & Q_{1} \leq Q_{2} \rightarrow Q_{1} \leq Q_{2} & =\leq \top,(208)\end{array}$

Australasian Journal of Logic (18:5) 2021, Article no. 4 


$$
\begin{array}{llll}
\alpha & (221) & Q_{1} \leq Q_{2} & (219),(220), \vee E, T C 3 \\
\top & (222) & R U Q U & (208),(221), \rightarrow I
\end{array}
$$

We take the following as a preferred form of $U Q U R$.

$$
\begin{array}{r}
\text { UQUR. } \quad X^{+} Q_{1}+R_{1} \cong X^{+} Q_{2}+R_{2} \wedge R_{1}<X^{+} \wedge R_{2}<X^{+} \rightarrow \\
Q_{1} \cong Q_{2} \wedge R_{1} \cong R_{2}
\end{array}
$$

\begin{tabular}{|c|c|c|c|}
\hline$\alpha$ & $(223)$ & $\begin{array}{l}X^{+} Q_{1}+R_{1} \cong X^{+} Q_{2}+R_{2} \wedge \\
R_{2}<X^{+}\end{array}$ & HYPOTHESIS \\
\hline$\rho$ & $(224)$ & $R_{1}<X^{+}$ & HYPOTHESIS \\
\hline$\iota$ & $(225)$ & $R_{2}<X^{+}$ & HYPOTHESIS \\
\hline$\alpha \wedge \iota$ & $(226)$ & $Q_{1} \leq Q_{2}$ & $\begin{array}{l}(223), D \cong,(225), \wedge E, \wedge I, \\
R U Q U\end{array}$ \\
\hline$\alpha \wedge \rho$ & $(227)$ & $Q_{2} \leq Q_{1}$ & (226), In Like Manner \\
\hline$\alpha \wedge \rho \wedge \iota$ & $(228)$ & $Q_{1} \cong Q_{2}$ & $(226),(227), \wedge I, D \cong$ \\
\hline$\alpha \wedge \rho \wedge \iota$ & $(229)$ & $R_{1}+X^{+} Q_{1} \cong R_{1}+X^{+} Q_{2}$ & $(228), Q M \cong \times, M \cong+$ \\
\hline$\alpha \wedge \rho \wedge \iota$ & $(230)$ & $R_{1}+X^{+} Q_{2} \cong R_{2}+X^{+} Q_{2}$ & $(229),(223), T R \cong$ \\
\hline$\alpha \wedge \rho \wedge \iota$ & $(231)$ & $R_{1} \cong R_{2}$ & $(230), M \cong+$ \\
\hline$\alpha \wedge \rho \wedge \iota$ & $(232)$ & $Q_{1} \cong Q_{2} \wedge R_{1} \cong R_{2}$ & $(228),(231), \wedge I$ \\
\hline$\top$ & $(233)$ & $U Q U R$ & $\begin{array}{l}\underset{\rightarrow I}{(223),}(224), \quad(225), \quad(232), \\
\end{array}$ \\
\hline$\omega$ & $(234)$ & $X^{+} Q_{1}+R_{1}=X^{+} Q_{2}+R_{2}$ & НYPOTHESIS \\
\hline$\omega$ & $(235)$ & $\alpha$ & $(234),(223),=\rightarrow \cong$ \\
\hline$\omega \wedge \rho \wedge \iota$ & $(236)$ & $Q_{1} \cong Q_{2}$ & (235), (228), REPLACEMENT \\
\hline T & $(237)$ & $\omega \wedge \rho \wedge \iota \rightarrow Q_{1} \cong Q_{2}$ & $(236), \rightarrow I$ \\
\hline 丁 & $(238)$ & $\omega \wedge \rho \wedge \iota \supset Q_{1}=Q_{2}$ & $(237), \cong \rightarrow \supset$ \\
\hline 丁 & $(239)$ & $\omega \wedge \rho \wedge \iota \supset R_{1}=R_{2}$ & $\begin{array}{l}\text { (238), IN LIKE Manner, } \\
\text { Use (231) }\end{array}$ \\
\hline$\top$ & $(240)$ & $\begin{array}{l}\omega \wedge \rho \wedge \iota \supset \\
\quad Q_{1}=Q_{2} \wedge R_{1}=R_{2}\end{array}$ & $(238),(239), D \supset, D \wedge \vee$ \\
\hline
\end{tabular}

Its derivation is as follows.

Unpacking the abbreviations, it will be noted that, after proving $U Q U R$ in (233), we have arrived in (240) in a wholly classical form of $U Q U R$ with Australasian Journal of Logic (18:5) 2021, Article no. 4 
ordinary equality. Using (233), (240), we get on moves already explored the following alternative forms.

$$
\begin{aligned}
& \text { UQUR1. } \quad P X \rightarrow . X Q+R \cong X Q_{1}+R_{1} \wedge R_{1}<X \wedge R<X \rightarrow \\
& Q \cong Q_{1} \wedge R \cong R_{1} \\
& \text { UQUR1. } \quad P X \rightarrow . R+X Q \cong R^{\star}+X Q^{\star} \wedge R<X \wedge R^{\star}<X \rightarrow \\
& Q \cong Q^{\star} \wedge R \cong R^{\star} \\
& \text { UQUR2. } P X \rightarrow . R+X Q \cong R^{\star}+X Q^{\star} \wedge R<X \wedge R^{\star}<X \supset \\
& Q=Q^{\star} \wedge R=R^{\star} \\
& \text { UQUR3. } X \neq 0 \wedge Y=R+X Q \cong Y=R_{1} X Q_{1} \wedge R<X \wedge R_{1}<X \supset \\
& Q=Q_{1} \wedge R=R_{1}
\end{aligned}
$$

We have by now proved quite a number of elementary theorems of $\mathrm{R}^{\sharp}$. Indeed, the material forms of all theorems in $\S 38, \S 39$ of [IMM] all hold in $\mathrm{R}^{\sharp}$, either because they have been directly demonstrated above or because they follow trivially from relevant counterparts demonstrated above.

However, the situation remains a bit puzzling. Lacking $\gamma$ in $R^{\sharp}$, so far as we know thus far, the best that we can do is to prove theorems of $C^{\sharp}$ one at a time. As the chain of reasoning which ended in UQUR3 (Kleene's $\star 146 b)$ shows, this can be quite a time-consuming business. First we need to get clear on what the relevant insights are; and then we have the task of transforming them to the classical vocabulary.

It would be tempting to shortcut this whole business, say by assuming $\gamma$ outright. However, no such shortcut is really satisfactory. In the first place, though it is always possible that eventually there will be no help for it, an outright assumption of $\gamma$ would have disastrous effects both on philosophical motivation and on the major technical results below. More important, producing a fair copy of Kleene is not really what we are after. The utility of trying to produce a fair copy of Kleene has lain mostly in the relevant results that we obtained by way of doing so, with honest arrows in them and with close attention to the kinds of distinction (e.g., in the relevant theory of order) to which that approach has led us. While in the end, we seek, and have sought, some approximation to intuitive reasoning, it has been necessary to keep careful formal track of how that reasoning turns out in the present context. While there has been neither space nor time to write out the above proofs in total detail-nor is there any reason to suppose that the reader would have read them in such detail-I have sought above to give

Australasian Journal of Logic (18:5) 2021, Article no. 4 
rather full indication of how a totally detailed proof might go.

In this matter, there is just no substitute for the reader's careful attention, irksome though that may be. In particular, the reader who has picked and chosen his way through the above proofs, and the information which accompanied them, ought to look again at some of them. In particular, he should attend carefully to the tags which the several steps bear, and he should ask himself how it came about that such a step can indeed be derived with the tag that it bears. For it is the tags, in this technique, which carry relevance, and it is in the tags that can be legitimately attached to a step and those which cannot be so attached that the principal innovations here lie; indeed, it is often in not being able to attach a tag relevantly that one comes to see what relevant arithmetical claim lies behind a familiar material one. Again, so far as we know, all the familiar material claims are made in $R^{\sharp}$; the difference lies in how they are made, and it is a most important difference when one comes to philosophize on what the natural numbers are and how we come to assert what we assert about them. We have, above, merely made a beginning. But it is a careful beginning, opening alternative interesting possibilities, both mathematical and philosophical. For their realization, a careful continuation is required.

\section{$\mathrm{X}$}

We have, in the preceding sections, proved a number of theorems of $C^{\sharp}$ on direct translation into $R^{\sharp}$. The question arises whether all theorems of $C^{\sharp}$ are thus provable. And the subsidiary question arises whether there exists a straightforward translation from $C^{\sharp}$ into $R^{\sharp}$ such that, on this translation, exactly the theorems of $C^{\sharp}$ are the theorems of $R^{\sharp}$.

We shall show in this section that the answer to the second question is "Yes." $C^{\sharp}$ is "hidden" in a clear and effective way. This has immediate import for the formal properties of $\mathrm{R}^{\sharp}$, since it means that the considerable amount of technical research into $C^{\sharp}$ is applicable to $R^{\sharp}$ as well. E.g., $R^{\sharp}$ is undecidable; it is incomplete; all recursive functions can be represented within it; certain formal statements that may be taken to express its consistency are unprovable within it; and so forth. (But these last remarks depend upon the assumption that $R^{\sharp}$ is consistent, while moreover requiring some care with respect to their exact formulation.)

However, it is not yet clear that the answer to the first question is "Yes";

Australasian Journal of Logic (18:5) 2021, Article no. 4 
i.e., that $R^{\sharp}$ contains $C^{\sharp}$ on the direct translation. If $\gamma$ holds, that question will be answered affirmatively. Accordingly, we shall be concerned in this section to think about various $\gamma$ related problems. But it should be pointed out that the direct translation from $C^{\sharp}$ to $R^{\sharp}$ is of independent interest, and that it is conceivable that this translation is exact even if $\gamma$ fails for all of $R^{\sharp}$.

To discuss translation in a fairly careful way, let us set up some conventions. We shall, accordingly, let $\mathscr{L}^{*}$ be the classical sublanguage of $\mathscr{L}^{\sharp}$; i.e., $\mathscr{L}^{*}$ is based on $\wedge, \sim, \forall X$, dropping $\rightarrow$ from among the primitives but otherwise building up $\mathscr{L}^{*}$ in the same way as $\mathscr{L}^{\sharp}$. Let $\mathrm{C}^{*}$ be the system got by restricting $C^{\sharp}$ to the $\mathscr{L}^{*}$ vocabulary; i.e., so far as its theorems are concerned, $C^{*}=C^{\sharp} \cap \mathscr{L}^{*}$. Obviously this involves no real restriction, since primitive $A \rightarrow B$ and defined $A \supset B$ are fully equivalent from the $C^{\sharp}$ viewpoint. Likewise, let $\mathrm{R}^{*}=\mathrm{R}^{\sharp} \cap \mathscr{L}^{*}$, in the same sense. Evidently this does involve a real restriction, since $A \rightarrow B$ and $A \supset B$ are sharply distinguished by $R^{\sharp}$.

By a translation from a language $\mathscr{L}$ to a language $\mathscr{L}^{\prime}$, we mean here simply a function $T$ defined on all formulas of $\mathscr{L}$ with values in $\mathscr{L}^{\prime}$. If $\mathscr{L} \subseteq \mathscr{L}^{\prime}$, and particularly if $\mathscr{L}=\mathscr{L}^{\prime}$, the idiot's translation is the function which takes every formula to itself; i.e., it is the identity function on $\mathscr{L}$. Where $\mathrm{S}$ and $\mathrm{S}^{\prime}$ are systems, based respectively on languages $\mathscr{L}$ and $\mathscr{L}^{\prime}$, we may also refer to a translation from $\mathscr{L}$ to $\mathscr{L}^{\prime}$ as a translation from $\mathrm{S}$ to $\mathrm{S}^{\prime}$. When convenient, we continue to indulge in the (theoretically incorrect) practice of identifying systems with the sets of their theorems.

Let $\mathrm{S}$ and $\mathrm{S}^{\prime}$ be systems, based respectively on languages $\mathscr{L}$ and $\mathscr{L}^{\prime}$, and let $T$ be a translation from $\mathbf{S}$ to $\mathbf{S}^{\prime}$. If $T$ preserves theoremhood in $\mathrm{S}$ (i.e., if for all $A \in \mathscr{L}$, if $A \in \mathrm{S}$ then $T(A) \in \mathrm{S}^{\prime}$ ), then $T$ is a good translation from $\mathrm{S}$ to $\mathrm{S}^{\prime}$. If $T$ preserves non-theoremhood in $\mathrm{S}$ (i.e., if for all $A \in \mathscr{L}$, if $T(A) \in \mathrm{S}^{\prime}$ then $A \in \mathrm{S}$ ), then $T$ is a fair translation from $\mathrm{S}$ to $\mathrm{S}^{\prime}$. Finally, $T$ is an exact translation from $\mathbf{S}$ to $\mathrm{S}^{\prime}$ if it preserves both theoremhood and non-theoremhood-i.e., if it is both fair and good. If there is an exact translation from $S$ to $S^{\prime}$, then clearly $S$ is "hidden in" $\mathrm{S}^{\prime}$ with respect to that translation. (Whether, however, this is a reasonable claim that $S$ is contained in $\mathrm{S}^{\prime}$ depends on the character of the translation $T$ itself-i.e., its simplicity, its effectivity, its semantic acceptability, and so forth.)

We may suppose $C^{\sharp}$ formulated axiomatically in a number of ways. The simplest is just to add $A \rightarrow B \rightarrow A$ (or even $\perp \rightarrow A$ ) as an additional axiom scheme to our formulation of $R^{\sharp}$. A second formulation takes all first-order tautologies in the language $\mathscr{L}^{\sharp}$ as logical axioms; in this case, it is readily 
observed that the rules $\rightarrow E, R M I$, and a simple rule $U G$ of generalization (from $A$, to infer $\forall X A$ ) suffice to yield all theorems of $C^{\sharp}$, with arithmetical axioms $A 11-A 18$. To formulate $C^{*}$ axiomatically, simply change all occurrences of $\rightarrow$ to $\supset$ in the axioms and rules of $C^{\sharp}$, trading in $\rightarrow E$ for $\supset E$ in particular.

We make some observations about idiots' translations.

Theorem 5. The idiot's translation from $\mathrm{R}^{\sharp}$ to $\mathrm{C}^{\sharp}$ is a good translation. The idiot's translation from $\mathrm{C}^{\sharp}$ to $\mathrm{R}^{\sharp}$ is a fair translation. Similarly, the idiot's translation from $\mathrm{R}^{*}$ to $\mathrm{C}^{*}$ is good, and from $\mathrm{C}^{*}$ to $\mathrm{R}^{*}$ is fair.

Proof. By definitions, given that we may formulate $C^{\sharp}$ from $R^{\sharp}$ by adding axioms.

We next define direct translation from $C^{\sharp}$ and $C^{*}$ to $R^{\sharp}$ and $R^{*}$. Specifically, the direct translation $*$ is defined as follows on all formulas of $\mathscr{L}^{\sharp}$ (and $a$ fortiori of $\left.\mathscr{L}^{*}\right)$.

(i) If $A$ is an atomic formula $T=U$, then $A^{*}$ is $A$

(ii) $\quad(B \wedge C)^{*}$ is $B^{*} \wedge C^{*}$

(iii) $(\sim B)^{*}$ is $\sim B^{*}$

(iv) $(B \rightarrow C)^{*}$ is $B^{*} \supset C^{*}$

(v) $(\forall X B)^{*}$ is $\forall X B^{*}$

Note that the direct translation, restricted to $\mathscr{L}^{*}$, coincides with the idiot's translation. Then

Theorem 6. The direct translation from $\mathrm{C}^{\sharp}$ to $\mathrm{C}^{*}$ is an exact translation. The direct translation from $\mathrm{C}^{\sharp}$ (or $\mathrm{C}^{*}$ ) to $\mathrm{R}^{*}$ (or $\mathrm{R}^{\sharp}$ ) is a fair translation.

Proof. Trivial, given Theorem 5.

We do not expect the idiot's translation from $C^{\sharp}$ to $R^{\sharp}$ to be exact. Rather, we anticipate that there will be some theorems of $C^{\sharp}$ which, in the vocabulary $\mathscr{L}^{\sharp}$, are not theorems of $\mathrm{R}^{\sharp}$, and our finitary consistency proof for $\mathrm{R}^{\sharp}$ will demonstrate that this is the case. But it would be nice if the direct translation from $C^{\sharp}$ to $R^{\sharp}$ were exact (or, what comes to the same thing, if $C^{*}$ and $\mathrm{R}^{*}$ have the same set of theorems). For this would mean that, so far as formalizing purely classical intuitions are concerned, $C^{\sharp}$ and $R^{\sharp}$ are on a par, 
in an appropriate sense. However, with the means presently at our disposal, we cannot demonstrate this exactness. We can, however, show the following.

Theorem 7. Suppose that $\gamma$ (i.e., $\supset E$, or equivalently $\Delta E$ ) is an admissible rule of $\mathrm{R}^{\sharp}$. Then the direct translation $*$ is an exact translation from $\mathrm{C}^{\sharp}$ to $\mathrm{R}^{\sharp}$, and $\mathrm{C}^{*}$ has the same theorems exactly as $\mathrm{R}^{*}$.

Proof. That $*$ is fair was shown in Theorem 6 . We need to show, on the supposition, that $*$ is also good. We defer this task.

Our interrupted Theorem 7 has, at any rate, a supposition, which may be true or false. And it would be nice to find an exact translation from $C^{\sharp}$ to $\mathrm{R}^{\sharp}$ whose exactness does not rest on such suppositions. The delta translation from $\mathscr{L}^{\sharp}$ to $\mathscr{L}^{*}$ (and hence to $\mathscr{L}^{\sharp}$ ) has this property, defining, for each formula $A$ of $\mathscr{L}^{\sharp}$, a formula $A \Delta$ as follows:

(vi) $A \Delta$ is $\Delta\left(A^{*}\right)$

Note that, for all formulas $B$ of $\mathscr{L}^{*}$,

(vii) $B \Delta$ is $\Delta B$

Before proving the exactness of the delta translation from $C^{\sharp}$ to $R^{\sharp}$, we note the following properties of $\Delta$ as reflected in theorem schemes of $R^{\sharp}$.

$$
\begin{array}{ll}
\triangle \triangle . & \triangle \triangle A \leftrightarrow \Delta A \\
\triangle \wedge . & \triangle(A \wedge B) \leftrightarrow \Delta A \wedge \triangle B \\
\Delta \vee . & \triangle(A \vee B) \leftrightarrow \Delta A \vee \triangle B \\
\Delta \forall . & \triangle \forall X A \leftrightarrow \forall X \triangle A \\
\triangle \exists . & \triangle \exists X A \leftrightarrow \exists X \triangle A \\
\triangle \supset E . & \triangle(B \supset C) \wedge \triangle B \rightarrow \triangle C \\
\triangle \supset I . & \triangle(B \supset C) \supset . \triangle B \supset \triangle C \\
\triangle \rightarrow I . & \nabla \triangle(B \supset C) \rightarrow . \nabla \triangle B \rightarrow \nabla \triangle C
\end{array}
$$

The first five of these, which are essentially distribution principles for $\triangle$, follow trivially from the definition of $\triangle A$ as $\perp \vee A$ and elementary distributive, confinement, and idempotence principles of the classical logical particles. But $\triangle \supset E$, which allows us to eliminate even material $\supset$ in $\Delta$ contexts, and $\triangle \supset I$, which allows us in $\Delta$ contexts to trade in little $\supset$ for big $\supset$ (and to Australasian Journal of Logic (18:5) 2021, Article no. 4 
take advantage of the fact that $\supset E$ is known to be admissible in $\left.\mathrm{R}^{\sharp}\right)$, are interesting enough to devote a derivation to. $\triangle \rightarrow I$, which allows us similarly to trade in $\supset$ for $\rightarrow$ in $\nabla \Delta$ contexts, is the main point behind $\triangle \supset I$, though we content ourselves with a derivation of $\triangle \supset I$ here.

\begin{tabular}{|c|c|c|c|}
\hline$\alpha$ & (1) & $\triangle(B \supset C) \wedge \triangle B$ & HYPOTHESIS \\
\hline$\alpha$ & $(2)$ & $\triangle(B \wedge \sim B \vee C)$ & $\triangle \wedge, D \wedge \vee$, Replacement \\
\hline$\alpha$ & $(3)$ & $\triangle \triangle C$ & $(2), \perp I$, Replacement,$D \triangle$ \\
\hline T & $(4)$ & $\triangle(B \supset C) \wedge \triangle B \rightarrow \triangle C$ & $(3), \triangle \triangle,(1), \rightarrow I$ \\
\hline$\rho$ & $(5)$ & $\nabla \triangle(B \supset C)$ & HYPOTHESIS \\
\hline$\iota$ & (6) & $\nabla \triangle B$ & HYPOTHESIS \\
\hline$\rho \wedge \iota$ & $(7)$ & $\triangle C$ & $(5), \nabla E,(6), \nabla E, \wedge I,(4), \rightarrow E$ \\
\hline$\rho \circ \iota$ & $(8)$ & $\triangle C$ & $(7), \wedge \simeq 0$, TAG REPLACEMENT \\
\hline$\top$ & $(9)$ & $\triangle(B \supset C) \supset . \triangle B \supset \triangle C$ & $(6),(8), \rightarrow I, D \supset,(5), \rightarrow I, D \supset$ \\
\hline
\end{tabular}

This completes the promised derivation, enabling us to show that the delta translation from $C^{\sharp}$ to $R^{\sharp}$ is exact.

Theorem 8. The following conditions are equivalent, for all formulas $A$ of $\mathscr{L}^{\sharp}$ :

(i) $A$ is a theorem of $\mathrm{C}^{\sharp}$

(ii) $\quad A \Delta$ is a theorem of $\mathrm{C}^{\sharp}$ (and indeed of $\mathrm{C}^{*}$ )

(iii) $A \Delta$ is a theorem of $\mathrm{R}^{\sharp}$ (and indeed of $\mathrm{R}^{*}$ )

Moreover, if $A$ is in the classical sublanguage $\mathscr{L}^{*}$, the following conditions are equivalent to each other and to each of (i)-(iii):

(iv) $A$ is a theorem of $\mathrm{C}^{*}$

(v) $\triangle A$ is a theorem of $\mathrm{C}^{*}$

(vi) $\triangle A$ is a theorem of $\mathrm{R}^{*}$

Accordingly, the translation $\Delta$ is exact from $\mathrm{C}^{\sharp}$ to $\mathrm{R}^{*}$.

Proof. If $A$ is in the sublanguage $\mathscr{L}^{*}$, each of the pairs (i)-(iv), (ii)-(v), (iii)(vi) is synonymous with the other, by definitions. Moreover, (iii) implies (ii), by Theorem 5. Assume that $\triangle\left(A^{*}\right)$ is a theorem of $C^{\sharp}$. Since $\perp \vee B$ and $B$ are classical equivalents for all $B$, evidently $A^{*}$, and hence $A$ (by Theorem

Australasian Journal of Logic (18:5) 2021, Article no. 4 
6 ) is a theorem of $C^{\sharp}$. So (ii) implies (i). We conclude the proof by showing that (i) implies (iii).

We suppose the second suggested formulation of $C^{\sharp}$ above, and we assume $A$ a theorem of $C^{\sharp}$. We prove that $\triangle\left(A^{*}\right)$ is a theorem of $\mathrm{R}^{\sharp}$, by induction on the length of proof of $A$.

First, suppose that $A$ is an axiom of $C^{\sharp}$. If it is a logical axiom, it is known from [ENT] that $A^{*}$ is a theorem of $\mathrm{R}^{\sharp}$, arguing at the $\mathrm{RQ}$ level; so then is $A \Delta$ by $\triangle I$. Moreover, each of the properly arithmetical axioms $A 11-A 18$ entail, in $\mathrm{R}^{\sharp}$, their $*$ translations, whence again $\triangle A^{*}$ is a theorem of $\mathrm{R}^{\sharp}$ for each such axiom of $C^{\sharp}$.

Next, suppose that $A$ comes in $C^{\sharp}$ by one of the rules $\rightarrow E, U G, R M I$, where on inductive hypothesis the premisses of this rule are theorems of $R^{\sharp}$ on the delta translation. Then the subcases are handled as follows:

Subcase $\rightarrow E$. $A$ comes in $C^{\sharp}$ from predecessors $B, B \rightarrow A$. On inductive hypothesis, $\triangle B^{*}$ and $\triangle\left(B^{*} \supset A^{*}\right)$ are theorems of $\mathrm{R}^{\sharp}$. By $\wedge I, \wedge \supset E, \rightarrow I$, so also is $\triangle A^{*}$, which was to be shown.

Subcase $U G$. $A$ is of the form $\forall X B$, coming by $U G$ from $B$. On inductive hypothesis, $\triangle B^{*}$ is a theorem of $\mathrm{R}^{\sharp}$, whence by $\forall I$ in $\mathrm{R}^{\sharp}$ so also is $\forall X \triangle B^{*}$. By $\triangle \forall$, Replacement, $\triangle \forall X B^{*}$ is also a theorem of $\mathrm{R}^{\sharp}$, which was to be shown.

Subcase $R M I . \quad A$ is of the form $A[X]$, and comes in $C^{\sharp}$ from predecessors $A[0]$ and $A[X] \rightarrow A\left[X^{\prime}\right]$. Noting that our substitution notation is preserved under translation, we may assume on inductive hypothesis that $\triangle A[0]^{*}$ and $\triangle A[X]^{*} \supset \triangle A\left[X^{\prime}\right]^{*}$ are theorems of $\mathrm{R}^{\sharp}$. By $\triangle \supset I, \supset E$, we have $\triangle A[X]^{*} \supset \triangle A\left[X^{\prime}\right]^{*}$ as a theorem of $\mathrm{R}^{\sharp}$ also. By $A 19^{\star \prime}$ of Section VII, with two applications of $\supset E$, we get as desired $\triangle A[X]^{*}$. This completes the inductive argument and with it the proof of Theorem 8 .

We may now complete the section by returning to our interrupted proof of Theorem 7 .

Proof of Theorem 7 (Completed). Suppose $A$ is a theorem of $\mathrm{C}^{\sharp}$. By Theorem $8, \triangle A^{*}$ is a theorem of $\mathrm{R}^{\sharp}$. By (unproved) supposition, $\gamma$ is admissible in $\mathrm{R}^{\sharp}$, whence $A^{*}$ is a theorem of $\mathrm{R}^{\sharp}$. Thus, on the supposition, $*$ is a good, and hence an exact, translation from $C^{\sharp}$ to $R^{\sharp}$, ending the proof of Theorem 7 .

Australasian Journal of Logic (18:5) 2021, Article no. 4 


\section{XI}

In this section, we apply the results of the last section to do some Gödelian metatheory for $R^{\sharp}$. In particular, we shall investigate the question whether, and in what sense, Gödel's famous theorems apply to $R^{\sharp}$. In making this investigation, and for the sake of simplicity, we shall assume the usual arithmetical mythology outright. Even so, many matters too tedious to work out will be simply left as conjectures.

In assuming the usual mythology, I assume in particular the standard model $\mathbb{N}=\left\langle\mathbf{N}, 0,{ }^{\prime},+, \times\right\rangle$ of the natural numbers, and that moreover all theorems of $C^{\sharp}$ are true therein on standard Tarskian interpretation. This assumption, which is of course stronger than those made in Gödel's seminal [FUS], implies outright that $C^{\sharp}$ is $\omega$-consistent, and a fortiori consistent. Since, moreover, $C^{\sharp}$ contains $\mathrm{R}^{\sharp}$ on the idiot's translation (though not conversely), $R^{\sharp}$ has these properties also. Moreover, I assume standard results of elementary recursion theory (to be found, e.g., in [IMM], though my terminology will not always agree with [IMM]).

It is immediate, on these assumptions, that

Theorem 9. $\mathrm{R}^{\sharp}$ is simply incomplete. I.e., there is a closed formula $A$ of $\mathscr{L}^{\sharp}$ such that neither $A$ nor $\sim A$ is a theorem of $\mathrm{R}^{\sharp}$.

Proof. $C^{\sharp}$ has this property, by Gödel's result, and non-theoremhood in $C^{\sharp}$ is preserved on the idiot's translation into $R^{\sharp}$, by Theorem 5 , ending the proof.

Theorem 10. $\mathrm{R}^{\sharp}$ is undecidable. I.e., there is no recursive procedure which, applied uniformly to the formulas of $\mathscr{L}^{\sharp}$, suffices to separate the theorems of $\mathrm{R}^{\sharp}$ from the non-theorems.

Proof. $C^{\sharp}$ is known to be undecidable, by Church! Suppose $\mathrm{R}^{\sharp}$ were nonetheless decidable. Applying this decision procedure to formulas $A \Delta$, we should get a decision procedure for $C^{\sharp}$ by Theorem 8 , which is impossible.

Theorem 11. $\mathrm{R}^{\sharp}$ is essentially undecidable. I.e., there is no decision procedure for any extension of $\mathrm{R}^{\sharp}$ which is consistent with respect to negation.

Proof. Let $\mathrm{S}^{\sharp}$ be an extension of $\mathrm{R}^{\sharp}$ for which a decision procedure exists. Let $S^{*}$ be the collection of theorems of $S^{\sharp}$ in the classical sublanguage $\mathscr{L}^{*}$, and let $\mathrm{S}^{\circ}$ be the class of formulas of $\mathrm{S}^{*}$ of the form $\triangle A$. We shall show

Australasian Journal of Logic (18:5) 2021, Article no. 4 
first that $\mathrm{S}^{\circ}$ contains $\triangle \perp$; i.e., $\perp \vee \perp$, whence $\mathrm{S}^{*}$ and hence $\mathrm{S}^{\sharp}$ contains $\perp$. But $S^{\sharp}$ contains $T$, which is already a theorem of $R^{\sharp}$. Accordingly, $S^{\sharp}$ is inconsistent with respect to negation. Contraposing, if the extension $S^{\sharp}$ of $\mathrm{R}^{\sharp}$ is negation-consistent, it is undecidable.

The argument for Theorem 11 is now complete, except for the demonstration that $\triangle \perp$ is indeed in $S^{\circ}$. Indeed, let $S$ be the set of formulas such that $A \in \mathrm{S}$ iff $\triangle A \in \mathrm{S}^{\circ}$. By Theorem 8, every theorem of $\mathrm{C}^{*}$ belongs to $\mathrm{S}$. Moreover, since we require of an extension of $\mathrm{R}^{\sharp}$ that it be closed under the rules of $R^{\sharp}$, it is clear (by the argument of Theorem 8, mutatis mutandis) that $\mathrm{S}$ is closed under $\supset E$ and $U G$. Accordingly, $\mathrm{S}$ is an extension of $\mathrm{C}^{*}$ in the classical sense. Moreover, applying our decision procedure to $S^{\circ}$ in particular, we get a decision procedure for $S$. So $S$ is a decidable classical extension of $\mathrm{C}^{*}$, in the vocabulary $\mathscr{L}^{*}$. There is only one such, namely the trivial theory, by Tarski, which is $\mathscr{L}^{*}$ itself. So $S$ contains $\perp$ in particular, whence $\mathrm{S}^{\circ}$ contains $\Delta \perp$ by definition of $\mathrm{S}$. This was what remained to be proved.

Corollary 1. Let $\mathrm{S}^{\sharp}$ be a decidable extension of $\mathrm{R}^{\sharp}$. Then $\mathrm{S}^{\sharp}$ is inconsistent.

Proof. To prove the theorem we proved the corollary, so that its proof is already complete.

Theorem 12. There is no recursively axiomatizable extension $\mathrm{T}^{\sharp}$ of $\mathrm{R}^{\sharp}$ such that, for every closed formula $A$ of $\mathscr{L}^{*}, A$ is a theorem of $\mathrm{T}^{\sharp}$ iff $A$ is true in the standard model. Accordingly, however truth is defined on $\rightarrow$ statements of $\mathscr{L}^{\sharp}$, the arithmetic truths remain relevantly unaxiomatizable.

Proof. Suppose that there is a recursively axiomatizable $\mathrm{T}^{\sharp}$ with the forbidden property. Let $\mathrm{T}^{*}$ be the class of theorems of $\mathrm{T}^{\sharp}$ in the vocabulary $\mathscr{L}^{*}$. Since $T^{\sharp}$ is recursively axiomatizable, and is the closure of its axioms under the deterministic rules of $R^{\sharp}, T^{\sharp}$ is recursively enumerable. So then is $T^{*}$, the class of arithmetic truths in the standard model. But this class is known not to be r.e., whence the theorem follows.

Theorem 13. Let $\mathbf{K}$ be an r.e. set of natural numbers. Then $\mathbf{K}$ is weakly expressible in $\mathrm{R}^{\sharp}$, in the following sense. There exists an open formula $A[x]$ of $\mathscr{L}^{\sharp}$, in the single free variable $x$, such that, for each natural number $I$,

(1) $I \in \mathbf{K}$ iff $A[I]$ is a theorem of $\mathrm{R}^{\sharp}$.

Australasian Journal of Logic (18:5) 2021, Article no. 4 
Conversely, if there is an $A[x]$ such that (1) holds for all I, the set $\mathbf{K}$ is recursively enumerable.

Proof. The last statement is trivial, since we may recursively enumerate the theorems of $\mathrm{R}^{\sharp}$, and those of the form $A[I]$ in particular, where $I \in \mathbf{N}$.

Suppose, then, that $\mathbf{K}$ is an r.e. set of natural numbers. Then, classically, there is an open formula $B[x]$ of $\mathscr{L}^{*}$ such that, for each $I \in \mathbf{N}$,

$\left(1^{\star}\right) \quad I \in \mathbf{K}$ iff $B[I]$ is a theorem of $\mathbf{C}^{*}$.

By Theorem 8, we get our desired $A[x]$ satisfying (1) by taking $A[x]$ as $\Delta B[x]$, completing the proof of Theorem 13.

Theorem 14. Let $\mathbf{K}$ be a recursive set of natural numbers. Then $\mathbf{K}$ is strongly expressible in $\mathrm{R}^{\sharp}$, in the following sense. There exists an open formula $A[x]$ of $\mathscr{L}^{\sharp}$ such that (1) holds for each natural number I, and moreover,

(2) $\quad I \notin \mathbf{K}$ iff $\sim A[I]$ is a theorem of $\mathrm{R}^{\sharp}$.

Conversely, if there is an $A[x]$ such that (1) and (2) hold, for all I, then $\mathbf{K}$ is recursive.

Proof. The last sentence is again trivial. Suppose then that $\mathbf{K}$ is recursive. Then, classically, there is an open formula $B[x]$ such that $\left(1^{\star}\right)$ holds for each natural number $I$, and moreover, for each $I$,

$\left(2^{\star}\right) \quad I \notin \mathbf{K}$ iff $\sim B[I]$ is a theorem of $\mathbf{C}^{*}$.

Let $A[x]$ be $\nabla \triangle B[x]$. Note that this is classically equivalent to $B[x]$. Accordingly, if $A[I]$ is a theorem of $\mathrm{R}^{\sharp}$, and hence by Theorem 5 a theorem of $\mathrm{C}^{\sharp}$, then $I \in \mathbf{K}$ by $\left(1^{\star}\right)$. Similarly, if $\sim A[I]$ is a theorem of $\mathrm{R}^{\sharp}, I \notin \mathbf{K}$ by $\left(2^{\star}\right)$. So both (1) and (2) are established from right to left, for arbitrary $I$. To show (2) from left to right, assume that $I \notin \mathbf{K}$. By $\left(2^{\star}\right), \sim B[I]$ is a theorem of $\mathrm{C}^{*}$. Trivially, $\nabla \sim B[I]$ is a theorem of $\mathrm{C}^{*}$. By Theorem $8, \Delta \nabla \sim B[I]$ is a theorem of $\mathrm{R}^{\sharp}$. But this is equivalent to $\sim \nabla \triangle B[I]$, i.e., to $\sim A[I]$, as promised. Finally, to show (1) from left to right, assume $I \in \mathbf{K}$. By $\left(1^{\star}\right)$ and Theorem $8, \triangle B[I]$ is a theorem of $\mathrm{R}^{\sharp}$, whence by $\nabla I$, and definition of $A[x]$, so is $A[I]$ a theorem of $R^{\sharp}$. This ends the proof of Theorem 13 .

So far, our use of classical results has been directly parasitic. It is perAustralasian Journal of Logic (18:5) 2021, Article no. 4 
haps a little more interesting, though scarcely more novel, actually to carry out some Gödel-style arguments, referred specifically to $\mathrm{R}^{\sharp}$. For example, enumerating effectively the class of formulas of $\mathscr{L}^{\sharp}$ in the single free variable $x$, and using $A_{I}[x]$ for the $I$ th such formula, let us establish Theorem 9 by direct argument, using our other results. Let $\mathbf{Q}$ be the set of natural numbers such that

(3) $\quad I \in \mathbf{Q}$ iff $A_{I}[I]$ is a theorem of $\mathrm{R}^{\sharp}$.

$\mathbf{Q}$ is clearly r.e., whence there is by Theorem 13 an open formula $Q[x]$ that weakly expresses Q. I.e., for all $I \in \mathbf{N}$,

(4) $Q[I]$ is a theorem of $\mathrm{R}^{\sharp}$ iff $A_{I}[I]$ is a theorem of $\mathrm{R}^{\sharp}$.

We consider now the formula $\sim Q[x]$. This is a formula in the single free variable $x$, and is accordingly $A_{J}[x]$ for some $J \in \mathbf{N}$, in view of our enumeration of such formulas. I.e., trivially,

(5) $\sim Q[J]$ is a theorem of $\mathrm{R}^{\sharp}$ iff $A_{J}[J]$ is a theorem of $\mathrm{R}^{\sharp}$.

While, since (4) holds for all $I$, we have in particular,

(6) $Q[J]$ is a theorem of $\mathrm{R}^{\sharp}$ iff $A_{J}[J]$ is a theorem of $\mathrm{R}^{\sharp}$.

So, by (5), (6),

(7) $\sim Q[J]$ is a theorem of $\mathrm{R}^{\sharp}$ iff $Q[J]$ is a theorem of $\mathrm{R}^{\sharp}$.

Having assumed $R^{\sharp}$ consistent, it immediately follows from (7),

(8) $\sim Q[J]$ is not a theorem of $\mathrm{R}^{\sharp}$, and

(9) $Q[J]$ is not a theorem of $\mathrm{R}^{\sharp}$.

So $Q[J]$ is an undecidable formula of $\mathrm{R}^{\sharp}$, which indeed we could patiently construct on Gödelian grounds were we so inclined. Moreover, we have by (8), (5), (3),

(10) $J \notin \mathbf{Q}$

Australasian Journal of Logic (18:5) 2021, Article no. 4 
whence we may conclude (if we have built $Q[x]$ familiarly),

$$
\sim Q[J] \text { is a true but unprovable closed formula of } \mathrm{R}^{\sharp} \text {. }
$$

This establishes Theorem 9 by direct argument (so to speak, since we haven't actually done much of the work). Henceforth, we let $\sim Q[J]$ be the Gödel formula ( $G \ddot{o} d$, for short). Let us devote at least as much argument to Theorem 10. If $R^{\sharp}$ were decidable, clearly the set $\mathbf{Q}$ above would be recursive. In view of Theorem 14, we could then choose $Q[x]$ above so that it satisfies not only the criteria (3)-(9), but also so that, for all $I \in \mathbf{N}$,

(12) $\quad I \notin \mathbf{Q}$ iff $\sim Q[I]$ is a theorem of $\mathrm{R}^{\sharp}$.

But then by (10), (12),

$$
\sim Q[J] \text { is a theorem of } \mathrm{R}^{\sharp}
$$

contradicting (8). For the truth of $G \ddot{o} d$ cannot be established by $\mathrm{R}^{\sharp}$ reason, even though we know by arithmetic faith (11) that $G \ddot{o} d$ is true. So Theorem 10 stands confirmed also by direct $R^{\sharp}$ argument.

Thus Gödel's famous first theorem, which asserts the incompleteness of effectively formulated arithmetics, goes through straightforwardly for $\mathrm{R}^{\sharp}$, together with a number of related theorems. This has been in no way surprising, given Theorem 8 and the strong assumptions that we have been making. Even under these strong assumptions, however, the status of Gödel's famous second theorem, which asserts that any system containing a suitable fragment of arithmetic can only be proved consistent by means stronger than those formulated by the system itself, is both more interesting and more dubious, relevantly speaking. Accordingly, we devote the next section to this and related topics. [TYPESCRIPT ENDS.]

\section{References}

[ETH] A.R. Anderson and N.D. Belnap. Enthymemes. Journal of Philosophy, 58(23):713-723, 1961.

[PCE] A.R. Anderson and N.D. Belnap. The pure calculus of entailment. Journal of Symbolic Logic, 27(1):19-52, 1962.

Australasian Journal of Logic (18:5) 2021, Article no. 4 
[ENT] A. R. Anderson and N. D. Belnap. Entailment, volume I. Princeton University Press, Princeton, 1975.

[IML] A. Church. Introduction to Mathematical Logic. Princeton University Press, Princeton, 1952.

[FML] H. B. Curry. Foundations of Mathematical Logic. McGraw Hill, New York, 1963.

[CRQ] J. M. Dunn, H. Leblanc, and R. K. Meyer. Completeness of relevant quantification theories. Notre Dame Journal of Formal Logic, 15(1):97$121,1974$.

[FUS] K. Gödel. Über formal unentscheidbare Sätze der Principia Mathematica und verwandter Systeme I. Monatshefte für Mathematik und Physik, 38:173-198, 1931.

[IMM] S. C. Kleene. Introduction to Metamathematics. North-Holland, New York, 1952.

[ERG] R. K. Meyer and J. M. Dunn. E, R, and $\gamma$. Journal of Symbolic Logic, 34(3):460-474, 1969.

[CR] R. K. Meyer. Coherence revisited. Unpublished monograph, 1973.

[IEN] R. K. Meyer. Intuitionism, entailment, negation. In H. Leblanc, editor, Truth, Syntax and Modality, pages 168-198. North-Holland, Amsterdam, 1973.

[NAR] R. K. Meyer. New axiomatics for relevant logics, I. Journal of Philosophical Logic, 3(1-2):53-86, 1974.

[IDP] R. K. Meyer. Idempotents in R. Zeitschrift fur mathematische Logik und Grundlagen der Mathematik, 32:25-30, 1986.

[ALI] R. K. Meyer and R. Routley. Algebraic analysis of entailment, I. Logique et Analyse, 15:407-428, 1972.

[ER] R. K. Meyer and R. Routley. Extensional reduction, I. The Monist, 60(3):355-369, 1977.

Australasian Journal of Logic (18:5) 2021, Article no. 4 
[PRL] J. Norman and R. Sylvan. Directions in Relevant Logic. Kluwer, Boston, 1989.

[ND] D. Prawitz. Natural Deduction: A Proof-Theoretical Study. Almqvist and Wiksell, Stockholm, 1965.

[TDE] W. V. O. Quine. Two dogmas of empiricism. The Philosophical Review, 60(1):20-43, 1951.

[SEI] R. Routley and R. K. Meyer. The semantics of entailment, I. In H. Leblanc, editor, Truth, Syntax and Modality, pages 199-243. NorthHolland, Amsterdam, 1973.

[SRL] A. Urquhart. Semantics for relevant logics. Journal of Symbolic Logic, 37(1):159-169, 1972.

Australasian Journal of Logic (18:5) 2021, Article no. 4 